\title{
The impact of dietary habits on cardiovascular risk factors and other metabolic parameters of free-living populations : methodological approaches to dietary analyses
}

Citation for published version (APA):

Pounis, G. (2014). The impact of dietary habits on cardiovascular risk factors and other metabolic parameters of free-living populations : methodological approaches to dietary analyses. [Doctoral Thesis, Maastricht University]. Maastricht University. https://doi.org/10.26481/dis.20140611gp

Document status and date:

Published: 01/01/2014

DOI:

10.26481/dis.20140611gp

Document Version:

Publisher's PDF, also known as Version of record

Please check the document version of this publication:

- A submitted manuscript is the version of the article upon submission and before peer-review. There can be important differences between the submitted version and the official published version of record. People interested in the research are advised to contact the author for the final version of the publication, or visit the DOI to the publisher's website.

- The final author version and the galley proof are versions of the publication after peer review.

- The final published version features the final layout of the paper including the volume, issue and page numbers.

Link to publication

\footnotetext{
General rights rights.

- You may freely distribute the URL identifying the publication in the public portal. please follow below link for the End User Agreement:

www.umlib.nl/taverne-license

Take down policy

If you believe that this document breaches copyright please contact us at:

repository@maastrichtuniversity.nl

providing details and we will investigate your claim.
}

Copyright and moral rights for the publications made accessible in the public portal are retained by the authors and/or other copyright owners and it is a condition of accessing publications that users recognise and abide by the legal requirements associated with these

- Users may download and print one copy of any publication from the public portal for the purpose of private study or research.

- You may not further distribute the material or use it for any profit-making activity or commercial gain

If the publication is distributed under the terms of Article 25fa of the Dutch Copyright Act, indicated by the "Taverne" license above, 
The impact of dietary habits on cardiovascular risk factors and other metabolic parameters of free-living populations: methodological approaches to dietary analyses 
(C) Georgios Pounis, Maastricht 2014

Thesis Maastricht University

ISBN: 978-960-93-6007-4

Layout:

Cover design by:

Printed by:
Georgios Pounis

Georgios Pounis

VOLCANO digital printing, Athens, Greece

All rights reserved. No part of this thesis may be reproduced, stored in a retrieval system or transmitted in any form or by any means, without the permission in writing from the author, or, when appropriate, of the publishers of the publications. 
The impact of dietary habits on cardiovascular risk factors and other metabolic parameters of free-living populations: methodological approaches to dietary analyses

\section{PROEFSCHRIFT}

ter verkrijging van de graad van doctor

aan de Universiteit Maastricht

op gezag van de Rector Magnificus,

Prof. dr. L. L. G. Soete,

Volgens het besluit van het College van Decanen,

In het openbaar te verdedigen op

11 June 2014, 10:00

door

Georgios Pounis 


\section{Promotoren}

Prof. dr. H. ten Cate

Prof. dr. G. de Gaetano, IRCCS Instituto Neurologico Mediterraneo Neuromed, Pozzilli (IS), Italy

Prof. dr. L. Iacoviello, IRCCS Instituto Neurologico Mediterraneo Neuromed, Pozzilli (IS), Italy

\section{Beoordelingscommissie}

Prof. dr. R.P. Koopmans, (chairman)

Dr. A.J. ten Cate-Hoek

Prof. dr. ir. P.C. Dagnelie

Dr. A. Siani, Institute of Food Sciences, National Research Council, Avellino, Italy Prof. dr. J. Vermylen, Katholieke Universiteit Leuven, Belgium

This thesis was printed with the generous support of my parents. 
$\mathcal{T}_{\mathcal{O}} \mathcal{M} y$ Family and $\mathcal{E l e f t h e r i a}$ 
Chapter 1. General Introduction 9-12

Chapters 2-7. Selected Publications 13-109

Chapter 8. General Discussion 110-114

Chapter 9. Conclusions 115-116

$\begin{array}{ll}\text { Chapter 10. Summary } & 117\end{array}$

$\begin{array}{lll}\text { Chapter 11. Curriculum vitae } & 118\end{array}$

Chapter 12. Publications 119-121

Chapter 13. Acknowledgements 122 


\section{Chapter 1. General Introduction}

Dietary habits have been strongly associated with primary and secondary prevention of several chronic diseases such as cardiovascular disease (CVD) and different types of cancer. The World Health Organization reports that non-healthy diet is one of the 3 most important risk factors that can be prevented, together with the lack of physical activity and smoking.

The evaluation of dietary habits can be performed at many levels leading to different types of public health conclusions. There are at least 4 different approaches, which are commonly used, in dietary analysis.

The first tries to measure the consumption of food groups that are usually used at the local "cuisine. This method can be applied using data from 24-hour dietary recalls, Food Frequency Questionnaires (FFQs) and diaries of food consumption. The most frequent conclusions of such analysis include the reporting of a possible positive or negative association of food groups' consumption with the studied disease. These inferences can help to better evaluate the importance of such food groups for public health perspectives.

The second type of dietary analysis goes quantitatively, one step further and using food composition tables to calculate the exact nutrient intake. This technique allows researchers to give specific guidelines to dieticians and related health providers about the ideal intake of nutrients in each disease or health condition. These inferences are of great importance for nutritionists especially in the process of editing specific dietary plans for a person, a patient or a group of people.

The third type of dietary analysis is based on a holistic evaluation of the dietary habits. A "dietary index" is the most common "a-priori" method of nutrition data management. This kind of analysis gives the opportunity to overall assess the diet and is useful in statistical analysis especially in models when it is appropriate to avoid the problem of co-linearity that dietary data have. The message to public health managers is very clear since they are informed about the percentage of people that have healthy dietary habits, so that they can easily plan their policies.

The last type of dietary analysis is also a holistic one but an "a-posteriori" method which succeeds to derive dietary patterns from the data of the sample or to discriminate dietary 
habits according to their impact on several diseases. Reduced Rank Regression (RRR) analysis, principal components analysis and discriminant analysis are the three major analyses, respectively, and they are performed using FFQs.

Besides the aforementioned 4 types of dietary analysis, valuable conclusions could also be extracted by studying dietary habits in a clinical environment. This could be considered as a fifth approach, based on the different nature of the data derived from the study design. The evidences extracted from this approach are considered as of great importance since the study approach limits the bias presented in studying associations between food or nutrients and health outcomes.

The five approaches in dietary analysis reported above introduce different types of scientific conclusions to research questions, in the level of food groups' consumption, nutrient intakes and dietary patterns adherence. However, intergraded nutritional analysis is of crucial importance especially when the association of dietary habits with chronic diseases such as CVD is studied.

Thus, this thesis will examine the complex impact of dietary habits on major cardiovascular risk factors and other metabolic parameters of free-living populations, using different methodological approaches to dietary analysis, in order to get more integrated conclusions.

Antioxidant vitamins and phytochemicals intake has been considered as crucially important in CVD prevention through different biological mechanisms. The overall assessment of antioxidants consumption in human diet seems important. An original article using data from the Moli-sani study analyzed and described the dietary index Food Antioxidant Content (FAC)-score (Chapter 2). The association of FAC-score with clinical biomarkers of CVD was also tested. By this way, the first and the third type of dietary analysis were used.

Health-related quality of life (HRQL) has been considered a valid predictor of CVD and overall mortality. Healthy eating seems to contribute to establishing a better quality of life. Thus, FAC-score was studied in the context of Mediterranean pattern adherence for its role in establishing better quality of life using data of the Moli-sani study (Chapter 3 ). The first and third dietary analyses were integrally used again. 
Mushroom, as a food group, could be considered as a good source of selenium, an important antioxidant component of the diet. The role of selenium on glucose levels is somehow controversial. Thus a third analysis conducted in the Moli-sani population aimed at evaluating the association of mushroom consumption and dietary selenium intake with blood glucose levels (Chapter 4). Such analyses concerning food group consumption (through mushroom intake) and nutrient intake (through dietary selenium) exhausted the first and second kind of dietary analysis mentioned in the thesis.

Folate status has been negatively associated with cerebrovascular disease, an important category of CVD. Differences in folate intake and status have been observed among EU countries. The evaluation of folate dietary intake as well as the extraction of dietary patterns associated with better folate status seems important. In the IMMIDIET population possible differences in folate status in two EU Countries (Italy and England) were assessed in relation to dietary habits and/or other lifestyle parameters (Chapter 5). By analyzing folate intake as a nutrient in relation to folate serum levels and by identifying a dietary pattern that accounts mostly for the variation in plasma folate, the second and the fourth type of dietary analysis were conducted.

A further study on the supplemental intake of folate as a single nutrient was performed using data from a randomized, double blind clinical trial. Major aim was to compare the efficacy of a diet particularly rich in natural folate compared with that of two different protocols of folic acid pharmacological supplementation, in subjects with "moderate" hyperhomocysteinemia (Chapter 6). Studying dietary habits in clinical environment fulfilled the use of the fifth type of dietary analysis in explaining the association of CVD risk factors and metabolic parameters with human diet.

Finally, the IMMIDIET study was used again to test the role of dietary habits on CVD using a holistic approach in dietary analysis. Growing evidence suggests that blood fatty acids (FAs) are major biomarkers/-predictors and even causal factors in many diseases, in particular cardiovascular disease. Differences in blood FAs profile among populations with different lifestyle have partly been attributed to differences in food intake. This analysis aimed at evaluating the main plasma and red blood cell FAs in three European populations and assessing the role of dietary patterns in explaining variations in their levels (Chapter 7). By 
the analysis of dietary patterns associated with better the $n-3$ fatty acids profile the fourth type of dietary analysis was conducted. 
Chapter 2.

Consumption of healthy foods at different content of antioxidant vitamins and phytochemicals and metabolic risk factors for cardiovascular disease in men and women of the Moli-sani study

Pounis G, Costanzo S, di Giuseppe R, de Lucia F, Santimone I, Sciarretta A, Barisciano P, Persichillo M, de Curtis A, Zito F, Di Castelnuovo AF, Sieri S, Benedetta Donati M, de Gaetano G, lacoviello L. Consumption of healthy foods at different content of antioxidant vitamins and phytochemicals and metabolic risk factors for cardiovascular disease in men and women of the Moli-sani study. Eur J Clin Nutr 2013; 67: 207-13. doi: 10.1038/ejcn.2012.201. 


\section{ORIGINAL ARTICLE}

\section{Consumption of healthy foods at different content of antioxidant vitamins and phytochemicals and metabolic risk factors for cardiovascular disease in men and women of the Moli-sani study}

G Pounis ${ }^{1}$, S Costanzo ${ }^{1}$, R di Giuseppe ${ }^{1}$, F de Lucia ${ }^{1}$, I Santimone ${ }^{1}$, A Sciarretta ${ }^{1}$, P Barisciano ${ }^{1}$, M Persichillo ${ }^{1}$, A de Curtis ${ }^{1}$, F Zito ${ }^{1}$, AF Di Castelnuovo ${ }^{1}$, S Sieri ${ }^{2}$, M Benedetta Donati ${ }^{1}, \mathrm{G}$ de Gaetano ${ }^{1}$ and L lacoviello ${ }^{1}$ on behalf of the Moli-sani Project Investigators

BACKGROUND/OBJECTIVES: To categorize healthy food groups into categories of low-antioxidant (LAC) or high-antioxidant vitamins and phytochemicals content $(\mathrm{HAC}$ ) and comparatively associate them with metabolic risk factors for cardiovascular disease (CVD).

SUBJECTS/METHODS: A total of 6879 women ( $55 \pm 12$ years) and 6892 men ( $56 \pm 12$ years) were analyzed from the Moli-sani cohort, randomly recruited from the general population. The European Prospective Investigation into Cancer and Nutrition Food Frequency Questionnaire was used for dietary assessment. The antioxidant content of each food group was evaluated using Istituto Nazionale di Ricerca per gli Alimenti e la Nutrizione and United States Department of Agriculture (USDA) food composition tables. Healthy foods, according to a Mediterranean dietary pattern, were categorized into HAC or LAC; total food antioxidant content (FAC) score was constructed for a comparative evaluation of the consumption of these two groups.

RESULTS: In men, an increase in FAC score, which represents an increased consumption of HAC with respect to LAC foods, was associated with a decrease in systolic blood pressure, diastolic blood pressure and C-reactive protein (CRP) $(\beta=-0.5, P=0.02$, $\beta=-0.3, P=0.02$ and $\beta=-0.03, P=0.03$, respectively). Logistic regression analyses showed that in men $15 \%$ (30 units) increase in FAC score was associated with $6 \%$ decrease in the likelihood of having hypertension (odds ratio $(\mathrm{OR})=0.94,95 \%$ confidence interval (CI) $0.91-0.98$ ) and $3 \%$ decrease in the likelihood of having a high CRP risk level ( $\mathrm{OR}=0.97,95 \% \mathrm{Cl} 0.94-0.99)$. No significant associations were observed in women.

CONCLUSIONS: A possible greater protective role of healthy HAC as compared with healthy LAC foods on hypertension and inflammation was detected in men. These results stress the importance of studying healthy foods according to their content in antioxidant vitamins and phytochemicals, in primary prevention of CVD.

European Journal of Clinical Nutrition (2013) 67, 207-213; doi:10.1038/ejcn.2012.201; published online 19 December 2012

Keywords: antioxidants; vitamins; phytochemicals; cardiovascular disease; inflammation

\section{INTRODUCTION}

The content in antioxidant vitamins and phytochemicals of a healthy diet such as the Mediterranean diet has long been recognized as beneficial for inflammation-related illnesses., ${ }^{1,2}$ Atherosclerosis progression is directly associated with the inflammation processes through several biological mechanisms, which are related to metabolic risk factors for cardiovascular disease (CVD) such as hypertension, hypercholesterolemia, hypertriglyceridemia and diabetes. ${ }^{3-5}$ Antioxidant vitamins and flavonoids contained in several foods were associated in epidemiological and clinical studies with a decrease in the oxidative stress caused by these risk factors. ${ }^{6-8}$

In particular, the oxidative stress reduction hypothesis of antioxidant vitamins and phytochemicals is supported by epidemiological and observational evidence that encouraged belief in and their use., ${ }^{9,10}$ Consumption of fruits and vegetables, rich in vitamin C and other antioxidant compounds, has been associated with a reduction in CVD mortality. ${ }^{11}$ These associations have been established through the anti-inflammatory properties of these nutrients depicted in the reduction of inflammatory biomarkers such as C-reactive protein (CRP), interleukin-6 and tumor necrosis factor alpha. ${ }^{12}$ In a US population survey, consumption of flavonoids was inversely related to CRP levels even after adjustment for total fruit and vegetable consumption. ${ }^{13}$

Antioxidant and phytochemical compounds are normally present in plant foods such as fruits, vegetables, vegetable oils and seeds, which represent the main food groups of the Mediterranean diet and have been recognized as protective against CVD and its risk factors. ${ }^{14,15}$ The Mediterranean dietary pattern may best fulfill requirements for an anti-inflammatory diet, at least in the Western world. ${ }^{12}$ However, the density of each food or food group in these healthy protective nutrients may differ. This may cause different impacts on CVD or on its risk factors prevention over the protective effect of healthy foods.

It is common for health operators to suggest patients to consume healthy foods, but in most cases their recommendations do not provide information about the antioxidant and phytochemicals content of such foods. This lack of information could

'Laboratory of Genetic and Environmental Epidemiology, Research Laboratories, Fondazione di Ricerca e Cura 'Giovanni Paolo II', Università Cattolica del Sacro Cuore, Campobasso, Italy and ${ }^{2}$ Nutritional Epidemiology Unit, National Cancer Institute, Milan, Italy. Correspondence: Professor L lacoviello, Laboratory of Genetic and Environmental Epidemiology, Research Laboratories, Fondazione di Ricerca e Cura 'Giovanni Paolo II', Università Cattolica del Sacro Cuore, Largo Gemelli 1, Campobasso 86100, Italy. E-mail: licia.iacoviello@moli-sani.org

Received 17 July 2012; revised 1 October 2012; accepted 8 October 2012; published online 19 December 2012 
induce the consumer to substitute healthy foods with a high antioxidant and phytochemicals content, with others healthy but low in such compounds. This could limit the protective effect that they could derive from a balanced proportion in the consumption of healthy food groups with high or low content in antioxidant vitamins and phytochemicals.

There is, indeed, lack of categorization of healthy foods according to their antioxidant content, which may impair their comparative analysis. As a consequence, there is limited evidence of a possible different impact of healthy foods on metabolic CVD risk factors due to their different antioxidant load.

The present study is aiming at categorizing healthy foods in groups at 'low-to-moderate' (LAC) or 'high antioxidant vitamins and phytochemicals content' (HAC) and comparatively evaluating their associations with metabolic risk factors for CVD.

\section{SUBJECTS AND METHODS}

\section{Study population}

The cohort of the Moli-sani Project was recruited in the Molise region (Italy) from city hall registries by a multistage sampling, which has previously been presented. ${ }^{16,17}$ Between March 2005 and April 2010, 24325 persons were recruited. Participants who had incomplete medical or dietary questionnaires $(n=1479)$ or were not caucasians or not born in Italy $(n=332)$ were excluded from the analysis. Furthermore, persons who had a history of CVD $(n=1719)$ or cancer $(n=889)$, or were taking drugs for diabetes, hypertension or hyperlipidemia $(n=6993)$ were excluded as these conditions may lead to changes in their usual diet. The final study sample that was included in this analysis was 13770 subjects $(6879$ men and 6891 women).

The Moli-sani project was approved by the Catholic University ethics committee. All participants provided written informed consent.

\section{Measurements and definition of risk factors}

Blood pressure $(\mathrm{mm} \mathrm{Hg})$ was measured by an automatic device (OMRONHEM-705CP) three times on the non-dominant arm, with the patient lying down for about $5 \mathrm{~min}$. The second and the third measurements were averaged. Weight and height were measured while the subjects wore no shoes and light underwear, and body mass index was calculated.

All blood samples were obtained from participants who had fasted overnight and had refrained from smoking for at least $6 \mathrm{~h}$. Serum lipids and blood glucose were assayed by enzymatic reaction methods using an automatic analyzer (ILab 350, Instrumentation laboratory (IL), Milan, Italy). Low-density lipoprotein cholesterol was calculated according to Friedewald. Hemocromocytometric analysis was performed by cell count (Coulter HMX, Beckman Coulter, IL Milan, Italy). High-sensitivity CRP was measured by a latex particle-enhanced immunoturbidimetric assay (IL Coagulation Systems on ACL9000). Inter- and intra-day CV were $5.5 \%$ and $4.17 \%$, respectively.

Subjects were classified as non-smokers if they had smoked less than 100 cigarettes in their lifetime or they had never smoked cigarettes, exsmokers if they had smoked cigarettes in the past and had stopped smoking for at least 1 year, and current smokers those who reported having smoked at least 100 cigarettes in their lifetime and still smoked or had quit smoking within the preceding year. ${ }^{18}$ Socio-economic status was defined as a score based on eight variables ranged from 0 to 8 ; the highest the score, the highest the level of socio-economic status. ${ }^{16}$ Physical activity was assessed by a structured questionnaire and expressed as daily energy expenditure in metabolic equivalent task hours. ${ }^{16,19}$

\section{Dietary assessment}

The European prospective investigation into cancer and nutrition-food frequency questionnaire specifically adapted for Italian population was used to determine usual nutritional intakes consumed in the past year. ${ }^{20}$ For the purpose of this study, as basic healthy food groups, using the Mediterranean pattern ${ }_{1}^{14}$ the following food types were included in the dietary analysis: leafy cooked vegetables, leafy raw vegetables, raw tomatoes, cooked tomatoes, root vegetables, cabbages, mushrooms, onions, legumes, citrus fruits, other fruits, nuts and seeds, olives, pasta and grains, chicken and turkey, fishes, olive oil, other vegetable oils, fruits and vegetable juices, low-fat milk, low-fat yogurt. The Nutritional Analysis of Food Frequency Questionnaires, National Cancer Institute, Milan, Italy
(NAF) software ${ }^{21}$ was used to transform information about food composition into intake of food items (g), energy (kcal) and micronutrients (g). The content in antioxidant vitamins and phytochemicals of each food group was studied using the Italian food composition tables of Istituto Nazionale di Ricerca per gli Alimenti e la Nutrizione. ${ }^{22}$ When data were missing, the food composition tables of United States Department of Agriculture USDA release 24 and USDA Database for flavonoid content of selected foods release 3 were used. ${ }^{23,24}$

The choice of the foods was firstly based on their healthiness and secondly on their content in antioxidant vitamins and phytochemicals. Coffee, chocolate and wine were not included in the analysis, despite their $\mathrm{HAC}$ as their healthiness was limited only to moderate consumption. ${ }^{25-27}$ As main antioxidant components of a healthy diet were considered

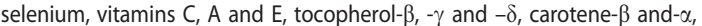
lycopene, lutein and all types of flavonoids. In order to categorize healthy food groups as at HAC or LAC, the content in all types of antioxidants components was standardized and summarized. The standardization formula was the $z=x-m / s$; where ' $z$ ' expressed the standardized concentration of each food group in each antioxidant component, ' $x$ ' expressed the concentration of each food group in each antioxidant component per $100 \mathrm{~g}$, ' $m$ ' was the mean concentration of each antioxidant component in all food groups and ' $s$ ' was the corresponding s.d. The standardization was used to avoid problems in summarizing results obtained from antioxidant vitamins and phytochemicals of different origin and type. The categorization in two groups was performed using the sum of the standardized content in antioxidant vitamins and phytochemicals of each food group and cutoff was the median of the summed standardized antioxidant content of each food group. Foods that had standardized antioxidant content over the median were categorized as HAC foods and the other as LAC. The distributions of all antioxidant compounds for all healthy food groups are illustrated in Table 1, together with the categorization to HAC and LAC.

After categorization of healthy food groups into these two categories, a total food antioxidant content (FAC) score was constructed. Theoretically, HAC food consumption should have greater protective role on metabolic CVD risk factors than LAC food intake, because of their content in antioxidant vitamins and phytochemicals; almost each participant consumed both food groups. Following this hypothesis individuals were divided into percentiles of consumption distribution for each of these two food groups, separately. Then HAC and LAC food consumption percentiles were scored from lower to higher with 1 to 100 and -100 to -1 , correspondingly. Finally, summing the two values for each participant the FAC score was derived ranging from -99 to 99 . People with greater values consumed mostly HAC foods, whereas individuals with lower score ate mostly LAC foods. Negative scoring was used to stress the possible replacement of LAC with HAC foods.

\section{Statistical analysis}

Normally distributed continuous variables are presented as mean \pm s.d., skewed as median (first, third quartiles) and categorical variables as frequencies. The normality of continuous variables was tested graphically. Comparisons of continuous variables between two groups of study were performed using the independent Student's $t$-test, for the normally distributed variables and the Mann-Whitney test, for the skewed. Associations between categorical variables were tested using the Pearson's $\chi^{2}$-test. Bonferroni correction for multiple comparisons (22 tests and significance level $a=0.05$ ) yield a corrected $a^{\prime}=0.002$, which indicated significant differences at the aforementioned univariate analysis.

Unadjusted and multiadjusted linear regression analyses were used to evaluate the association between antioxidant compound intake and metabolic CVD risk factors. Each of the continuous HAC, LAC, vitamin C, E, $A$ and $\beta$-carotene intake and FAC score was associated using linear regression models with systolic (SBP) and diastolic blood pressure (DBP), total cholesterol, low-density lipoprotein, high-density lipoprotein, triglycerides, CRP and glucose levels. Normality of residuals, homoscedasticity and multiple co-linearity were evaluated by plotting standardized residuals against the predicted values. To better evaluate the impact of FAC score on hypertension and CRP levels 2 logistic regression models were used. The first binary logistic regression model had as main outcome the presence of hypertension. The major independent factor was FAC score and the model was adjusted for the same confounders as the regression models. Deviance residuals evaluated goodness-of-fit. The second ordinal logistic regression model had as main outcome CRP levels categorized in the three risk levels for CVD derived from the American Heart Association and US Centers for 
Table 1. Content of healthy food groups in most common antioxidant compounds and its categorization to 'High' or 'Low Antioxidant Content' $(\text { HAC or LAC })^{\mathrm{a}}$

\begin{tabular}{|c|c|c|c|c|c|c|c|c|c|c|c|c|}
\hline & \multicolumn{12}{|c|}{ Common antioxidants compounds per $100 \mathrm{gr}$} \\
\hline & $\begin{array}{l}\text { Selenium } \\
(\mu g)\end{array}$ & $\begin{array}{l}\text { Vitamin C } \\
(\mathrm{mg})\end{array}$ & $\begin{array}{l}\text { Vitamin } A \\
\text { (IU) }\end{array}$ & $\begin{array}{l}\text { Vitamin } E \\
(m g)\end{array}$ & $\begin{array}{c}\text { Tocopherol- } \beta \\
(\mathrm{mg})\end{array}$ & $\begin{array}{c}\text { Tocopherol- } \gamma \\
(m g)\end{array}$ & $\begin{array}{c}\text { Tocopherol- } \delta \\
\text { (mg) }\end{array}$ & $\begin{array}{c}\text { Carotene- } \beta \\
(\mu g)\end{array}$ & $\begin{array}{c}\text { Carotene- } \alpha \\
(\mu g)\end{array}$ & $\begin{array}{c}\text { Lycopene } \\
(\mu g)\end{array}$ & $\begin{array}{l}\text { Lutein } \\
(\mu g)\end{array}$ & $\begin{array}{l}\text { flavonoids } \\
\text { (mg) }\end{array}$ \\
\hline $\begin{array}{l}\text { High antioxidant content } \\
\text { Leafy vegetables, }\end{array}$ & 1.00 & 15.47 & 8504 & 1.39 & - & 0.14 & 0.01 & 5082 & - & - & 7159 & - \\
\hline $\begin{array}{l}\text { Leafy vegetables, } \\
\text { raw }\end{array}$ & 0.93 & 56.70 & - & 0.93 & - & 0.25 & - & 1902 & - & - & 8918 & 9.56 \\
\hline Tomatoes, raw & - & 21.00 & - & 1.03 & 0.01 & 0.12 & - & 252 & 101 & 2573 & 123 & 1.48 \\
\hline Tomatoes, cooked & 0.50 & 22.80 & 489 & 0.56 & 0.01 & 0.21 & 0.01 & 293 & - & 3041 & 94 & 0.78 \\
\hline $\begin{array}{l}\text { Root vegetables, } \\
\text { raw }\end{array}$ & 0.40 & 5.50 & 16706 & 0.46 & 0.01 & 0.27 & - & 6888 & 3477 & 1 & 256 & 0.60 \\
\hline Citrus fruits & 0.67 & 44.00 & - & 0.21 & - & - & - & 178 & 40 & - & 92 & 20.64 \\
\hline Nuts and seeds & 5.80 & 0.70 & - & 2.00 & 0.20 & 15.02 & 0.83 & 62 & - & - & 390 & 4.57 \\
\hline Fishes & 35.40 & 0.53 & 80 & 0.38 & - & - & - & - & - & - & - & - \\
\hline Olive oil & - & - & - & 51.30 & 0.11 & 0.83 & - & - & - & - & - & - \\
\hline $\begin{array}{l}\text { Fruits and } \\
\text { vegetables juices }\end{array}$ & 0.24 & 21.00 & 3962 & 0.48 & - & - & - & 2842 & 870 & 1808 & 108 & 5.09 \\
\hline \multicolumn{13}{|c|}{ Low-to-moderate antioxidant content } \\
\hline Cabbages & 0.83 & 47.60 & - & 0.85 & - & 0.08 & - & 488 & 11 & - & 380 & 1.39 \\
\hline Mushrooms, raw & 11.90 & 3.00 & - & 0.12 & - & - & - & - & - & - & - & - \\
\hline Onions, raw & 0.25 & 5.00 & - & 0.12 & - & - & - & 15 & - & - & 10 & 15.36 \\
\hline Legumes, cooked & 2.00 & 1.35 & 4 & 0.07 & - & - & - & - & - & - & - & 5.44 \\
\hline Other fruits & 0.06 & 8.00 & - & 0.34 & - & 0.02 & - & 85 & 4 & - & 73 & 15.32 \\
\hline Olives & 0.90 & 2.00 & - & 1.99 & - & - & - & 309 & - & - & 510 & \\
\hline Pasta and grains & 13.27 & -.00 & 19 & 0.11 & - & - & - & - & - & - & 16 & - \\
\hline Chicken and Turkey & 22.47 & - & 214 & 0.27 & - & - & - & - & - & - & - & - \\
\hline Vegetable oils & - & - & - & 27.69 & - & - & - & - & - & - & - & - \\
\hline Skimmed milk & 3.10 & 1.00 & 67 & 0.04 & - & - & - & 10 & - & - & - & - \\
\hline Skimmed yogurt & 3.30 & 1.00 & 33 & 0.06 & - & - & - & 5 & - & - & - & - \\
\hline
\end{tabular}

The Italian INRAN food composition tables were used. When data was missing the USDA food composition tables release 24 and USDA Database for the flavonoid content of selected foods release 3 were also used. The choice of the foods was performed with the main concern to match them with those that are usually consumed in the Italian cooking habits.

Disease Control and Prevention ${ }^{28}((1)<1 \mathrm{mg} / \mathrm{l}$ low risk (2) $1-3 \mathrm{mg} / \mathrm{l}$ intermediate risk, (3) $>3 \mathrm{mg} / \mathrm{l}$ high risk). The major independent factor was FAC score. The proportional odds assumption was tested graphically. Twosided $P$ value $<0.05$ was considered as statistically significant. STATA version 9 software was used for all calculations (STATA Corp., College Station, TX, USA).

\section{RESULTS}

Table 2 shows the distribution of socio-demographic, lifestyle, dietary and clinical characteristics of men and women. Men were older, had greater levels of physical activity and worse smoking habits than women $(P$ for all $<0.001)$. Furthermore, men had higher values than women $(P$ for all $<0.001)$ of the majority of metabolic risk factors for CVD except for cholesterol and highdensity lipoprotein that were higher in women and for CRP levels that showed no difference. Men had greater intake of antioxidant vitamin $E$ than women. In addition they consumed greater amounts of both HAC and LAC foods $(P$ for all $<0.001)$ and had a higher FAC score than women ( $P$ for $t$-test $<0.001)$.

Tables 3 and 4 illustrate results from both unadjusted and multiadjusted regression analyses for men and women separately, with metabolic risk factors for CVD as dependent variables and antioxidant foods or vitamins as independent factors. In men, HAC food consumption was negatively associated with SBP and DBP in multiadjusted analysis ( $\beta$-coef $=-0.5, P=0.01$ and $\beta$-coef $=$ $-0.3, P<0.001$, respectively), whereas LAC food intake was not $(P>0.05)$. FAC score increase, which represents the increase in HAC compared with LAC food intake, was associated with a decrease in both SBP and DBP $(\beta$-coef $=-0.5, P=0.02$ and $\beta$ coef $=-0.3, P=0.02$, respectively). Multiadjusted analysis in men also revealed negative associations of $L A C, H A C$ food intake and FAC score with log-CRP $(\beta$-coef $=-0.02, P=0.01, \beta$-coef $=$ $-0.02, P=0.03$ and $\beta$-coef $=-0.03, P=0.03$, respectively). However, none of the three antioxidant dietary factors was associated with lipid profile biomarkers or with glucose levels $(P$ for all $\geqslant 0.05)$.
Antioxidant vitamin food content, considered separately, was in general not associated with metabolic risk factors for CVD. Only vitamin $E$ content was negatively associated with log-triglyceride and -CRP $\quad(\beta$-coef $=-0.02, \quad P<0.001 \quad$ and $\beta$-coef $=-0.02$, $P<0.001$, respectively) and $\beta$-carotene content was negatively associated with SBP, DBP and log-triglyceride $(\beta$-coef $=-0.15$, $P<0.001, \quad \beta$-coef $=-0.06, P=0.01, \beta$-coef $=-0.003, P=0.01$, respectively).

In women, both unadjusted and multiadjusted regression analyses suggested no significant association of antioxidant dietary factors with metabolic CVD risk factors (Table 4).

Binary logistic regression analysis in men after adjustment for age, physical activity, current smoking habits, social status, energy intake, red wine, chocolate and coffee consumption showed that $15 \%$ (30 units) increase in FAC score was associated with $4 \%$ decrease in likelihood of having hypertension (OR for 30 units increase $=0.96,95 \% \mathrm{Cl}$ 0.93-0.99). Ordinal logistic regression adjusted for the same confounders showed that 15\% (30 units) increase in FAC was associated with 3\% decrease in likelihood of having higher CRP risk level (OR for 30 units increase $=0.97,95 \%$ $\mathrm{Cl}$ 0.94-0.99).

\section{DISCUSSION}

The protective role of antioxidant compounds included in a healthy dietary pattern such as Mediterranean diet on blood pressure levels and hypertension has been already established in epidemiological studies and clinical trials. ${ }^{6,8,29}$ Vegetarians, whose antioxidant vitamins and phytochemicals consumption is particularly high, tend to have lower blood pressure than nonvegetarians. ${ }^{30,31}$ Our findings extend such knowledge by stressing that the choice of healthy foods should also be focused on their antioxidant load, especially in men.

Our study showed that in men, the consumption of healthy foods with high rather than low content in antioxidant vitamins and phytochemicals was associated with lower levels of blood 
Table 2. Socio-demographic, dietary and clinical characteristics of men and women studied

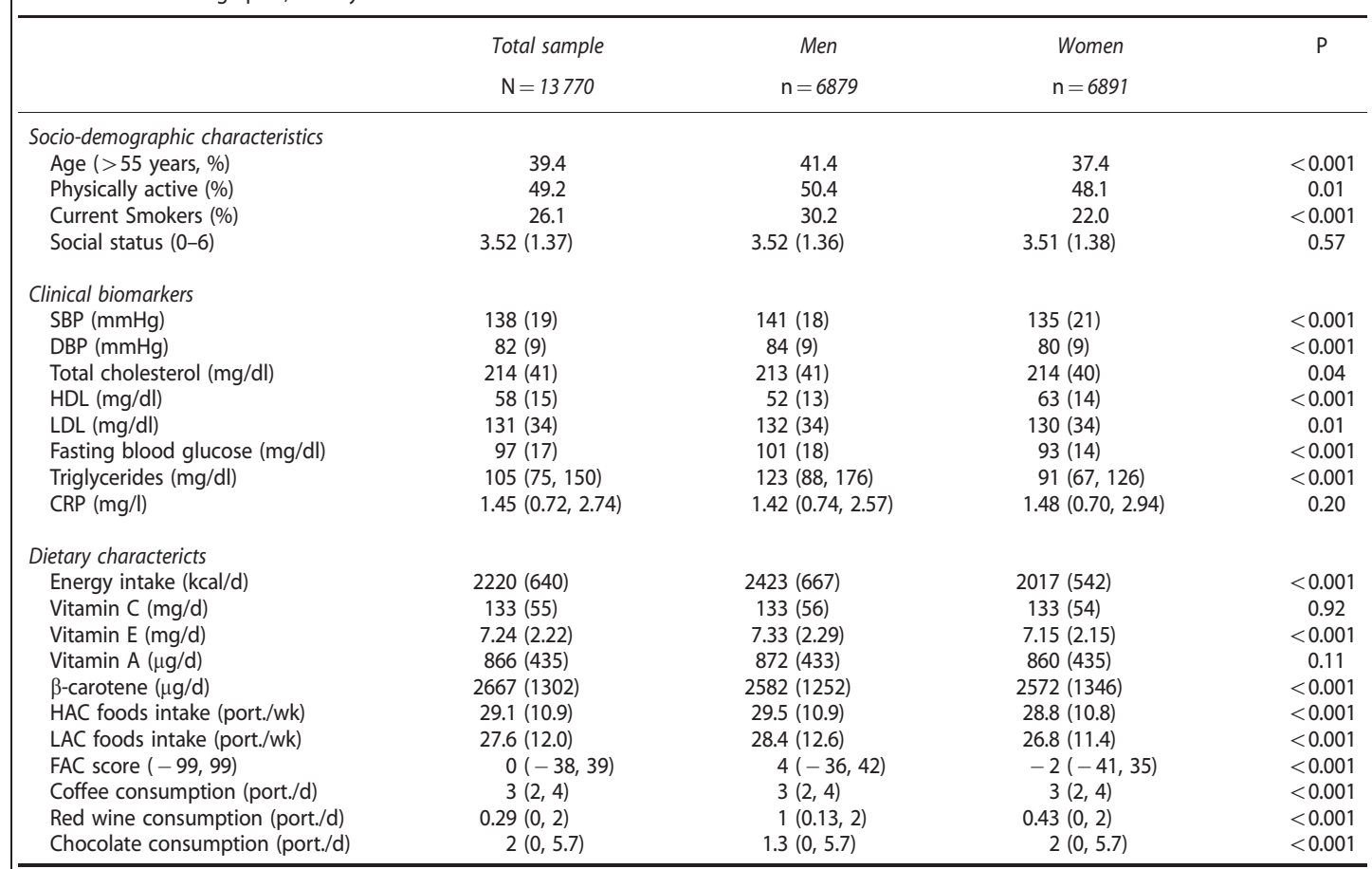

Abbreviations: CRP, C-reactive protein; DBP, diastolic blood pressure; FAC, food antioxidant content; HAC, high-antioxidant content; HDL, high-density lipoprotein; LAC, low-antioxidant content; LDL, low-density lipoprotein; SBP, systolic blood pressure. Continuous data are presented as mean (s.d.) for normally distributed and as median (first, third quartiles) for skewed. Results for continuous data are derived through comparisons between groups using Student's $t$-test for normally distributed and Mann-Whitney's test for skewed. Categorical data are presented as frequencies and P-values derived through Pearson's $\chi^{2}$-test.

pressure and of the inflammation marker CRP, whereas these associations did not appear in women.

The use of FAC score indicated that the increase in consumption of HAC with respect to LAC foods is also associated with a lower likelihood of having hypertension. In particular, 15\% (30 units) increase in FAC score was associated with $6 \%$ decrease in likelihood of having hypertension after controlling for most common confounders.

Although multiple factors affect the presence and development of hypertension, the pathogenesis of this disease is related to excessive oxidative stress. Excessive local superoxide production in kidneys, central nervous system and vasculature, along with inflammatory activation, could result in hypertension in animal models. ${ }^{32}$ Studies in rats showed the development of hypertension with associated increases in oxidative stress and impaired vasodilation after exposure to a high-salt- and oxidantcontaining diet. ${ }^{33}$ Further, infusion of superoxide dismutase lowered oxidative stress and blood pressure in these animal models. ${ }^{34-36}$ These data indicate, regardless of etiology, that the excessive reactive oxygen species form a central common pathway, by which disparate influences may induce and exacerbate hypertension. Antioxidant vitamin- and phytochemical-rich diets that include selenium, vitamins $C, A$ and $E$, tocopherol- $\beta,-\gamma$ and $-\delta$, carotene- $\beta$ and- $\alpha$, lycopene, lutein and all type of flavonoids may reduce the excessive oxidative stress with protective effects on hypertension.

We have also observed an anti-inflammatory effect of choosing HAC instead of LAC food groups, on the bases of the negative association between FAC score and CRP levels in men. In particular, 15\% (30 units) increase in FAC score was associated with 3\% decrease in likelihood of having increased CRP risk level as defined by the American Heart Association recommendations, using CRP levels. ${ }^{28}$ Several population-based observational or randomized clinical trials have reported that CRP levels are inversely associated with dietary intake of fruits and vegetables which are rich in antioxidant vitamins and phytochemicals. ${ }^{17,37}$ Also, Chun et al. in the National Health and Nutrition Examination Survey study 1999-2002 conducted in USA revealed that the intakes of fruits, vegetables and flavonoid compounds was inversely associated with serum CRP concentrations after adjusting for covariates. ${ }^{13}$

All observed associations of HAC instead of LAC food consumption with markers of CVD risk were present only in men. Recent studies have discussed the different level of oxidative stress present in men and women. In particular, whole-body reactive oxygen species production seemed to be higher in healthy young men than in women..$^{38}$ Under healthy conditions, cellular respiration in the mitochondria is the dominant source of reactive oxygen species. Therefore, a higher baseline metabolic rate in men than in women ${ }^{39}$ might contribute to a higher level of oxidative stress in men..$^{38}$ In addition, the biological response of premenopausal women to oxidative stress regulation may be peculiar. ${ }^{38}$ These mechanisms have already been hypothesized as a possible rational basis for differences in the propensity of the two genders in developing atherosclerosis. They may explain the sensitivity to antioxidants of men compared with women, which was observed in the present study.

Furthermore, in both genders, antioxidant vitamins were not associated with most of CVD-related risk factors. Although a significant body of epidemiological and clinical data indicate that 
Healthy eating, antioxidants and metabolic risk factors

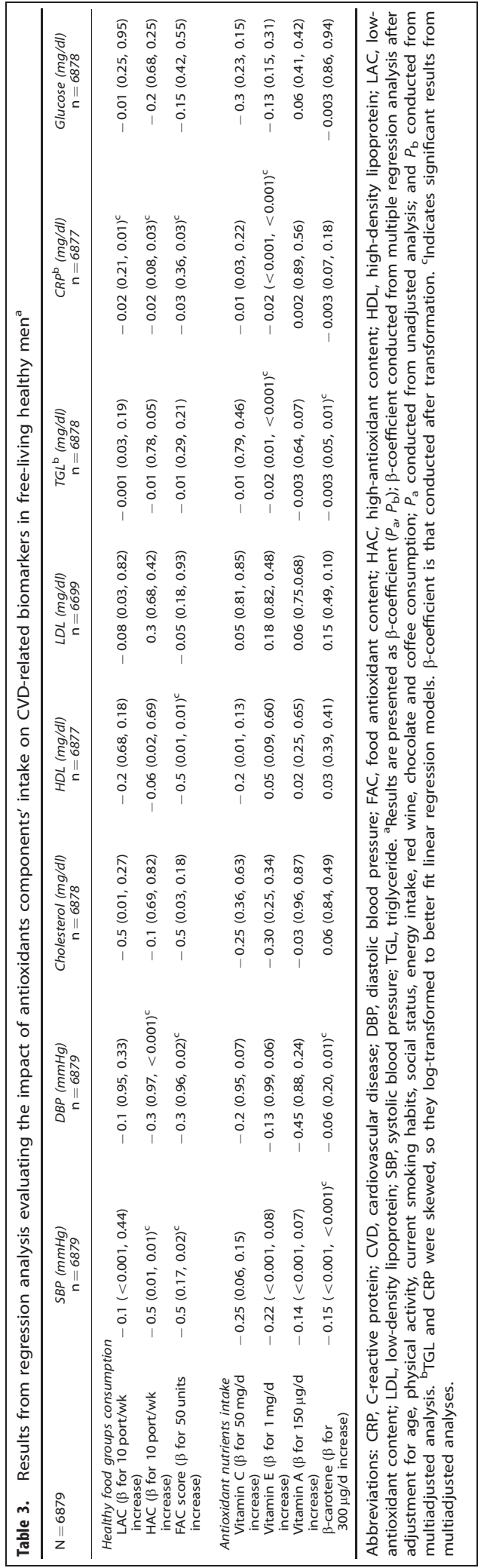

c) 2013 Macmillan Publishers Limited

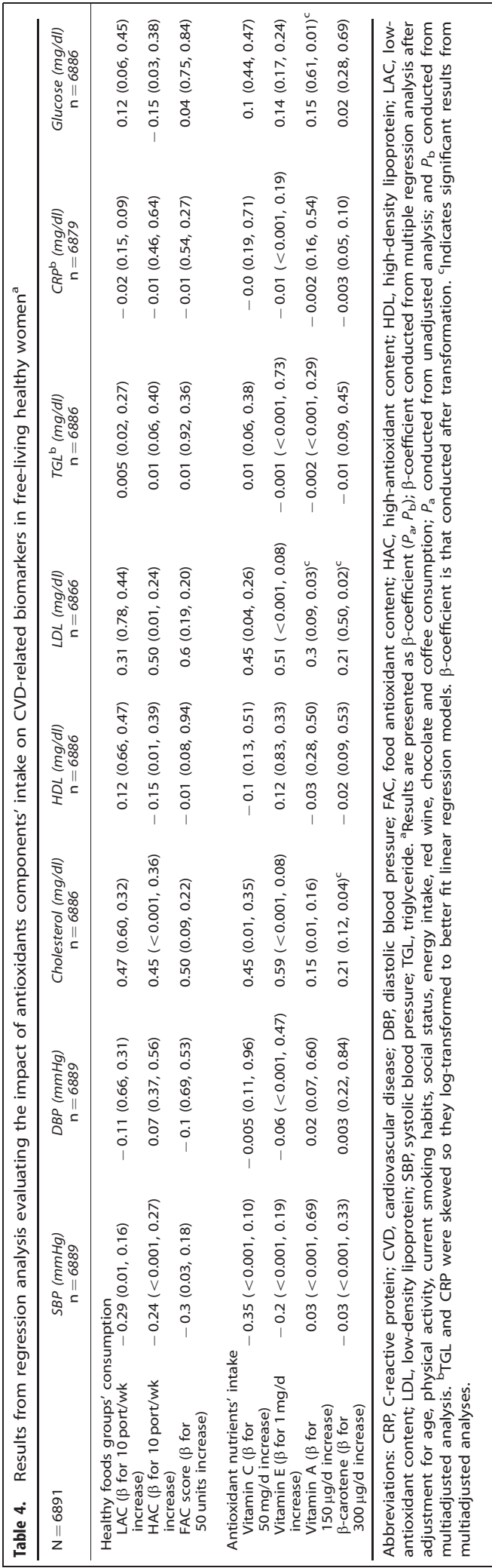

European Journal of Clinical Nutrition (2013) 207-213 
antioxidant-rich diets reduce cardiovascular risk factors, randomized trials and population studies using natural antioxidants have yielded disappointing results. ${ }^{40}$ The reasons behind this lack of efficacy are not completely clear; however, it could be suggested that only a combination of many antioxidant vitamins and phytochemicals as those contained in natural foods can protect against inflammation processes and CVD-related clinical risk factors. 6,40

Focusing on the significant results of this study, it is of great importance to stress that the choice of healthy foods should also include as a criterion their total antioxidant content. The foods included in the HAC category in the present dietary analysis are leafy vegetables, tomatoes, citrus fruit, nuts and seeds, mixed salads, fishes and vegetable or fruit juices. However, both healthy food groups, high or low in antioxidant content, should be consumed to establish healthy dietary habits. There are several nutrients other than antioxidants contained in such foods, which could improve health of population. Some epidemiological evidence have stressed that nutritional education is directly associated with the choice of healthy foods. ${ }^{41}$ Thus, efforts should be made in public health promotion for the improvement of nutritional education to let people make more informed choices in the way of a balanced consumption of healthy foods.

Beyond the relevance of the findings of the present work, some limitations still exist. First, its cross-sectional nature does not enable determination of causality. To rule out the possibility that the associations found were dependent on either changes in lifestyle (particularly in dietary habits) as a consequence of a disease or the presence of less healthier food intake in healthy people, we had preliminarily excluded from our analyses all subjects with previous CVD or cancer, or with diagnosed diabetes, hypertension or hyperlipidemia. Moreover, the dietary information was retrieved only once and, thus, may be prone to recall bias and seasonal variation.

In conclusion, this study showed that in a sample of free-living apparently healthy men the choice of foods high rather than low in antioxidant content may reduce blood pressure and inflammation. This indicates the need for public health promotion strategies focused on a balanced choice of healthy foods high in antioxidant content. Also, our results stress the importance of studying healthy foods according to their antioxidant content, in primary prevention of CVD.

\section{CONFLICT OF INTEREST}

The authors declare no conflict of interest.

\section{ACKNOWLEDGEMENTS}

We thank Associazione Cuore-Sano (Campobasso, Italy), IL Instrumentation-Laboratory (Milano, Italy), Derby-Blue (San Lazzaro di Savena, Bologna, Italy), Caffè Monforte (Campobasso, Italy) and Sepinia SpaA (Sepino, Italy) for their support to the MOLISANI project. The Moli-sani cohort recruitment was supported by an unrestricted grant from Pfizer Foundation (Rome, Italy) and the Italian Ministry of University and Research (MIUR, Rome, Italy), Programma Triennale di Ricerca, Decreto no.1588.

\section{REFERENCES}

1 Di Giuseppe R, Arcari A, Serafini M, Di Castelnuovo A, Zito F, De Curtis A et al. Total dietary antioxidant capacity and lung function in an Italian population: a favorable role in premenopausal/never smoker women. Eur J Clin Nutr 2012; 66: 61-68.

2 De Pablo P, Dietrich T, Karlson EW. Antioxidants and other novel cardiovascular risk factors in subjects with rheumatoid arthritis in a large population sample. Arthritis Rheum 2007; 57: 953-962.

3 Ross R. Atherosclerosis: an inflammatory disease. N Engl J Med 1999; 340: 115-126.

4 Hansson GK. Inflammation, atherosclerosis, and coronary artery disease. N Engl J Med 2005; 352: 1685-1695.
5 Ridker PM, Hennekens $\mathrm{CH}$, Buring JE, Rifai N. C-reactive protein and other markers of inflammation in the prediction of cardiovascular disease in women. $N$ Engl J Med 2000; 342: 836-843.

6 Kizhakekuttu TJ, Widlansky ME. Natural antioxidants and hypertension: promise and challenges. Cardiovasc Ther 2010; 28: 20-32.

7 Dauchet L, Amouyel P, Hercberg S, Dallongeville J. Fruit and vegetable consumption and risk of coronary heart disease: a meta-analysis of cohort studies. J Nutr 2006; 136: 2588-2593.

8 Appel LJ, Moore TJ, Obarzanek E, Vollmer WM, Svetkey LP, Sacks FM et al. A clinical trial of the effects of dietary patterns on blood pressure. DASH Collaborative Research Group. N Engl J Med 1997; 336: 1117-1124.

9 Rimm EB, Stampfer MJ, Ascherio A, Giovannucci E, Colditz GA, Willett WC. Vitamin E consumption and the risk of coronary heart disease in men. N Engl J Med 1993; 328: $1450-1456$

10 Stanner SA, Hughes J, Kelly CNM, Buttriss J. A review of the epidemiological evidence for the 'antioxidant hypothesis'. Public Health Nutr 2004; 7 407-422.

11 Verlangieri AJ, Kapeghian JC, El-Dean S, Bush M. Fruit and vegetable consumption and cardiovascular mortality. Med Hypotheses 1985; 16: 7-15.

12 Galland L. Diet and inflammation. Nutr Clin Pract 2010; 25: 634-640

13 Chun OK, Chung SJ, Claycombe KJ, Song WO. Serum C-reactive protein concentrations are inversely associated with dietary flavonoid intake in U.S. adults. Jutr 2008; 138: 753-760.

14 Trichopoulou A, Costacou T, Bamia C, Trichopoulos D. Adherence to a Mediterranean diet and survival in a Greek population. N Engl J Med 2003; 348: 2599-2608.

15 Sofi F, Cesari F, Abbate R, Gensini GF, Casini A. Adherence to Mediterranean diet and health status: meta-analysis. BMJ 2008; 337: 1344

16 lacoviello L, Bonanni A, Costanzo S, De Curtis A, Di Castelnuovo A, Olivieri M et al. The Moli-Sani Project, a randomized, prospective cohort study in the Molise region in Italy, design, rationale and objectives. Ital J Public Health 2007; 110-118.

17 Centritto F, lacoviello L, di Giuseppe R, De Curtis A, Costanzo S, Zito F et al. Dietary patterns, cardiovascular risk factors and C-reactive protein in a healthy Italian population. Nutr Metab Cardiovasc Dis 2009; 19: 697-706.

18 Nuorti JP, Butler JC, Farley MM, Harrison LH, McGeer A, Kolczak MS et al. Cigarette smoking and invasive pneumococcal disease. Active Bacterial Core Surveillance Team. N Engl J Med 2000; 342: 681-689.

19 Ainsworth BE, Haskell WL, Whitt MC, Irwin ML, Swartz AM, Strath SJ et al. Compendium of physical activities: an update of activity codes and MET intensities. Med Sci Sports Exerc 2000; 32(Suppl): 498-504.

20 Pisani $P$, Faggiano F, Krogh V, Palli D, Vineis P, Berrino F. Relative validity and reproducibility of a food frequency dietary questionnaire for use in the Italian EPIC centers. Int J Epidemiol 1997; 26: 152-160.

21 Pala V, Sieri S, Palli D, Salvini S, Berrino F, Bellegotti M et al. Diet in the Italian EPIC cohorts: presentation of data and methodological issues. Tumori 2003; 89: 594-607.

22 Italian food composition tables. Istituto Nazionale di Ricerca per gli Alimenti e la Nutrizione. http://www.inran.it/646/tabelle_di_composizione_degli_alimenti. html, Accessed 20 December 2011.

23 USDA National Nutrient Database for Standard Reference, Release 24. US Department of Agriculture, Agricultural Research Servicehttp://www.ars.usda.gov/ ba/bhnrc/ndlAccessed 20 December 2011.

24 USDA Database for the Flavonoid Content of Selected Foods Release 3. U.S. Department of Agriculture Agricultural Research Service. http://www. ars.usda.gov/SP2UserFiles/Place/12354500/Data/Flav/Flav_R03.pdf, Accessed 20 December 2011.

25 Di Giuseppe R, Di Castelnuovo A, Centritto F, Zito F, De Curtis A, Costanzo S et al. Regular consumption of dark chocolate is associated with low serum concentrations of C-reactive protein in a healthy Italian population. J Nutr 2008; 138 1939-1945.

26 Di Castelnuovo A, Costanzo S, Donati MB, lacoviello L, de Gaetano G. Prevention of cardiovascular risk by moderate alcohol consumption: epidemiologic evidence and plausible mechanisms. Intern Emerg Med 2010; 5: 291-297.

27 Wu JN, Ho SC, Zhou C, Ling WH, Chen WQ, Wang CL et al. Coffee consumption and risk of coronary heart diseases: a meta-analysis of 21 prospective cohort studies. Int J Cardiol 2009; 137: 216-225.

28 Pearson TA, Mensah GA, Alexander RW, Anderson JL, Cannon 3rd RO, Criqui M et al. Markers of inflammation and cardiovascular disease: application to clinical and public health practice: A statement for healthcare professionals from the Centers for Disease Control and Prevention and the American Heart Association. Circulation 2003; 107: 499-511.

29 Parslow RA, Sachdev P, Salonikas C, Lux O, Jorm AF, Naidoo D. Associations between plasma antioxidants and hypertension in a community-based sample of 415 Australians aged 60-64. J Hum Hypertens 2005; 19: 219-226.

30 Rouse IL, Beilin L, Armstrong BK, Vandongen R. Blood-pressure-lowering effect of a vegetarian diet: controlled trial in normotensive subjects. Lancet 1983; 1: 5-10. 
31 Margetts $B M$, Beilin LJ, Vandongen R, Armstrong BK. Vegetarian diet in mild hypertension: a randomised controlled trial. BMJ 1986; 293: 1468-1471.

32 Harrison DG, Gongora MC. Oxidative stress and hypertension. Med Clin North Am 2009; 93: 621-635.

33 Miyagawa K, Ohashi M, Yamashita S, Kojima M, Sato K, Ueda R et al. Increased oxidative stress impairs endothelial modulation of contractions in arteries from spontaneously hypertensive rats. J Hypertens 2007; 25: 415-421.

34 Nakazono K, Watanabe N, Matsuno K, Sasaki J, Sato T, Inoue M. Does superoxide underlie the pathogenesis of hypertension? Proc Natl Acad Sci 1991; 88: 10045-10048. 35 Laursen JB, Rajagopalan S, Galis Z, Tarpey M, Freemam BA, Harrison DG. Role of superoxide in angiotensin II-induced but not catecholamine-induced hypertension. Circulation 1997; 95: 588-593.

36 Schnackenberg CG, Welch WJ, Wilcox CS. Normalization of blood pressure and renal vascular resistance in SHR with a membrane-permeable superoxide dismutase mimetic: role of nitric oxide. Hypertension 1998; 32: 59-64.
37 Esmaillzadeh A, Kimiagar M, Mehrabi Y, Azadbakht L, Hu FB, Willett WC. Fruit and vegetable intakes, C-reactive protein, and the metabolic syndrome. Am J Clin Nutr 2006; 84: 1489-1497.

38 Ide T, Tsutsui H, Ohashi N, Hayashidani S, Suematsu N, Tsuchihashi M et al. Greater oxidative stress in healthy young men compared with premenopausal women. Arterioscler Thromb Vasc Biol 2002; 22: 438-442.

39 Meijer GA, Westerterp KR, Saris WH, ten Hoor F. Sleeping metabolic rate in relation to body composition and the menstrual cycle. Am J Clin Nutr 1992; 55: 637-640.

40 Kris-Etherton PM, Lichtenstein AH, Howard BV, Steinberg D, Witztum JL. Antioxidant vitamin supplements and cardiovascular disease. Circulation 2004; 110 637-641.

41 Pounis G, Makri S, Gougias L, Makris H, Papakonstantinou M, Panagiotakos D et al. Consumer perception and use of iron fortified foods is associated with their knowledge and understanding of nutritional issues. Food Qual Pref 2011; 22 683-688. 
Chapter 3.

Adherence to a Mediterranean diet is associated with a better healthrelated quality of life: a possible role of high dietary antioxidant content

Bonaccio M, Di Castelnuovo A, Bonanni A, Costanzo S, De Lucia F, Pounis G, Zito F, Donati MB, de Gaetano G, lacoviello L; Moli-sani project Investigators. Adherence to a Mediterranean diet is associated with a better health-related quality of life: a possible role of high dietary antioxidant content. BMJ Open 2013; 3(8). pii: e003003. doi: 10.1136/bmjopen-2013-003003. 


\title{
BMJ Adherence to a Mediterranean diet is Open associated with a better health-related quality of life: a possible role of high dietary antioxidant content
}

\author{
Marialaura Bonaccio, ${ }^{1,2}$ Augusto Di Castelnuovo, ${ }^{1}$ Americo Bonanni, ${ }^{1,3}$ \\ Simona Costanzo, ${ }^{1}$ Francesca De Lucia, ${ }^{1}$ George Pounis, ${ }^{1}$ Francesco Zito, ${ }^{1}$ \\ Maria Benedetta Donati, ${ }^{2}$ Giovanni de Gaetano, ${ }^{2}$ Licia lacoviello, ${ }^{2,4}$ \\ on behalf of the Moli-sani project Investigators*
}

To cite: Bonaccio M, Di Castelnuovo A, Bonanni A, et al. Adherence to a Mediterranean diet is associated with a better health-related quality of life: possible role of high dietary antioxidant content. BMJ Open 2013:3:e003003. doi:10.1136/bmjopen-2013003003

- Prepublication history and additional material for this paper is available online. To view these files please visit the journal online (http://dx.doi.org/10.1136 bmjopen-2013-003003).

*The Moli-sani Project Investigators are listed in the online supplementary appendix.

Received 5 April 2013 Revised 20 June 2013 Accepted 2 July 2013

For numbered affiliations see end of article.

Correspondence to Dr Licia lacoviello; licia.iacoviello@moli-sani.org

\section{ABSTRACT}

Objectives: Mediterranean diet (MD) is associated with a reduced risk of major chronic disease. Healthrelated quality of life (HRQL) is a valid predictor of mortality. The aim of this study is to investigate the association between MD and HRQL and to examine the possible role of dietary antioxidants, fibre content and/or fatty acid components.

Design: Cross-sectional study on a sample of Italian participants enrolled in the Moli-sani Project, a population-based cohort study. Food intake was recorded by the Italian European prospective investigation into cancer and nutrition study food frequency questionnaire. Adherence to MD was appraised by a Greek Mediterranean diet score (MDS), an Italian Mediterranean diet index (IMI) and by principal component analysis (PCA). HRQL was assessed by the 36-Item Short Form Health Survey. Setting: Molise region, Italy.

Participants: 16937 participants of 24325 Italian citizens (age $\geq 35$ ).

Main outcomes: Dietary patterns and HRQL.

Results: Menta/ health was associated consistently and positively with MDS, IMI and an 'Olive oil and vegetable' pattern (PCA1), but negatively with an 'Eggs and sweets' pattern (PCA3). Physical health was associated positively with MDS and PCA1, but negatively with a 'Meat and pasta' pattern. Subjects with the highest MD adherence had $42 \%$ (MDS), 34\% (IMI) or 59\% (PCA1) statistically significant multivariable odds of being in the uppermost level of mental health, as compared with subjects in the lowest category. The associations disappeared after further adjustment for either total food antioxidant content or dietary fibre, while they were not modified by the inclusion of either monounsaturated or polyunsaturated fatty acids. Individuals in the highest PCA1 or PCA3 had significantly higher odds of being in the top level of physical health.

Conclusions: Adherence to an MD pattern is associated with better HRQL. The association is stronger with mental health than with physical health. Dietary total antioxidant and fibre content independently explain this relationship.

\section{ARTICLE SUMMARY}

\section{Article focus}

- Investigating the association between adherence to the Mediterranean diet and health-related quality of life.

- Examining the possible role of dietary antioxidants, fibre intake and/or fatty acid components in explaining the association.

\section{Key messages}

- Mental health is positively associated with a Mediterranean diet and inversely linked to 'Eggs and sweets' dietary pattern.

- The association is mainly accounted for not only by the total antioxidant content but also by the fibre dietary content.

- Fatty acid compounds do not explain the association between the Mediterranean diet and health-related quality of life.

Strengths and limitations of this study

- This study is apparently the first to provide a likely account of dietary antioxidants for the direct association between the Mediterranean diet and health-related quality of life.

- For the first time, this topic was addressed by using simultaneously two a priori Mediterranean scores and the a posteriori dietary patterns obtained by principal component analysis.

- The present study was cross-sectional and shares all the limitations of this study type; in particular, the inference of possible causality is unwarranted.

\section{INTRODUCTION}

Mediterranean diet (MD) is a healthy eating pattern associated with reduced risk for cardiovascular and neurodegenerative diseases and some types of cancer. ${ }^{1-3}$ This dietary pattern is characterised by the wide consumption of plant foods, cereals, legumes, fish and olive oil as the main source of fat 
and moderate red wine consumption. The biological mechanisms for chronic disease prevention associated with an MD pattern are high amounts of antioxidants, polyphenols and other compounds such as monounsaturated and polyunsaturated fatty acids. ${ }^{4-7}$

Health-related quality of life (HRQL) refers to an individual's subjective evaluation of his own health and wellbeing $^{8}$ and has rapidly become an important issue in modern times since Western societies are facing a process of increasing population ageing. Self-perceived health status is closely associated with cardiovascular disease (CVD) and all-cause mortality and many studies have found that self-health ratings are important predictors of mortality for persons with cardiovascular disease, ${ }^{9}{ }^{10}$ middle-aged subjects ${ }^{11}$ and young adults. ${ }^{12}$ So far, few studies have investigated the relationship between MD and self-rated health in a general population.

Recent evidence suggests a positive correlation between adherence to MD and HRQL, ${ }^{13} 14$ not only in adults but also in adolescents. ${ }^{15}$ Further evidence has recently supported the relationship between MD and depression showing a potential protective role of MD against the onset of depressive symptoms. ${ }^{16} 17$

One of the most accredited hypotheses is that MD is positively associated with better overall health status and reduced risk of major chronic diseases because of its high content of different beneficial compounds, such as antioxidants, largely present in leafy vegetables, fruits, olive oil and red wine, monounsaturated (olive oil) or polyunsaturated fatty acids (mainly from fish and nuts ${ }^{5}$ ), dietary fibre ${ }^{18} 19$ or low glycaemic index. ${ }^{20}$

Our study aimed to test the association of MD and other dietary patterns with mental and physical health to examine the possible role played by total food antioxidant content (FAC), dietary fibre and monounsaturated or polyunsaturated fatty acids.

\section{SUBJECTS AND METHODS \\ Study population}

The study population consisted of participants in the Moli-sani Project, a population-based cohort study which randomly recruited 24325 citizens of Molise, a region placed between central and southern Italy. Between March 2005 and April 2010, men and women aged $\geq 35$ years were randomly recruited from subjects included in the city-hall registries of Molise. ${ }^{21}$ Exclusion criteria were pregnancy, disturbances in understanding/ willing processes, ongoing poly traumas or coma and refusal to sign the informed consent form.

After exclusion of subjects reporting a personal history of cardiovascular disease (angina, myocardial infarction, heart failure, revascularisation procedures and stroke; $5.7 \%$ ), cancer $(3.1 \%)$ or of those for whom there was no information available on health-related quality of life $(21.7 \%)$ or dietary habits $(3.9 \%), 16937$ subjects were finally included in the analysis presented.
Comparison between the sample considered in the present study ( $\mathrm{n}=16937)$ and excluded participants $(n=7388)$ showed a substantial homogeneity for sex $(p=0.23)$. The sample included was younger $(53 \pm 10.8$ vs $62 \pm 12.1 ; \quad \mathrm{p}<0.0001)$ and had higher social status $(p<0.0001)$, income $(p<0.0001)$ or education $(p<0.0001)$. Potential selection biases could emerge since the 36-Item Short Form Health Survey (SF-36) questionnaire was selfadministered and the topic not easily understandable by the elderly or people with a lower educational level or social status. In addition, we excluded subjects with CVD or cancer, which are medical conditions related to ageing and somehow to social status.

\section{Dietary information}

The validated Italian European prospective investigation into cancer and nutrition study food frequency questionnaire was used to evaluate food intake. ${ }^{22} 23$ The questionnaire, computerised with tailor-made software, allowed participants to be interviewed in an interactive way, by including illustrations of sample dishes of definite size or by reference to standard portion size. To simplify interpretation of data and to minimise within-person variations in intakes of individual foods, 188 food items were classified into 45 predefined food groups on the basis of similar nutrient characteristics or culinary usage (see online supplementary appendix 2 ).

Moderate alcohol intake was defined as regularly drinking no more than one drink a day by women, and no more than two drinks a day for men.

Food consumption patterns were generated by using Principal Components Analysis (PCA) conducted on the correlation matrix of 45 food groups. ${ }^{24}$ Three main factors emerged, in agreement with previous findings in the same population. ${ }^{24}$ The pattern identified as 'Olive Oil and Vegetables' was characterised by high positive loadings for olive oil, vegetables, legumes, soups, fruits and fish. The pattern named 'Meat and pasta' was characterised by high positive loadings for pasta, cooked tomatoes, red meat, animal fats and alcoholic beverages, and negative loadings of breakfast cereals and yogurt. The 'Eggs and Sweets' pattern was characterised by high positive loadings for eggs, margarines, processed meat and sugar and sweets.

We evaluated the adherence to MD by using the MD score (MDS) elaborated by Trichopoulou et al. ${ }^{25}$ Scoring was based on the intake of the following nine items: vegetables, legumes, fruit and nuts, dairy products, cereals, meat and meat products, fish, alcohol, and the ratio of monounsaturated:saturated fat. For most items, consumption above the study median received 1 point; all other intakes received 0 point. For dairy products, meat and meat products, consumption below the median received 1 point. Medians are gender specific. For ethanol, men who consumed $10-50 \mathrm{~g} /$ day and women who consumed $5-25 \mathrm{~g}$ /day received 1 point; otherwise, the score was 0 . The possible scores ranged between 0 and 9 , the latter reflecting the maximal adherence. 
We also used a newly proposed Italian Mediterranean diet index (IMI) whose score is based on the intake of 11 items: high intake of six typical Mediterranean foods (pasta; typical Mediterranean vegetables such as raw tomatoes, leafy vegetables, onion and garlic, salad, and fruiting vegetables; fruit; legumes; olive oil and fish); low intake of four non-Mediterranean foods (soft drinks, butter, red meat and potatoes) and alcohol consumption. If consumption of typical Mediterranean foods was in the third tertile of the distribution, the person received 1 point; all other intakes received 0 point. If consumption of non-Mediterranean foods was in the first tertile of the distribution, the person received 1 point. Ethanol received 1 point for intake up to $12 \mathrm{~g} /$ day; abstainers and persons who consumed $>12 \mathrm{~g} /$ day received 0 points. Possible scores ranged from 0 to $11 .^{26}$ Such an Italian index was conceived to better capture healthy eating including foods more typically consumed in Italy.

The total FAC score was used to measure the antioxidant content from the diet. Its construction was already described in a previous study conducted within the Moli-sani cohort. ${ }^{27}$ Briefly, the content in the antioxidant vitamins and phytochemicals of each food group was derived by using the food composition tables from the Istituto Nazionale di Ricerca per gli Alimenti e la Nutrizione and the US Department of Agriculture (USDA). Healthy foods, according to an MD pattern, were categorised into either high or low antioxidant content. The total FAC score was constructed for a comparative evaluation of the consumption of these two groups. Coffee, chocolate and wine were not included in the analysis, despite their high antioxidant content, as their healthiness is reportedly limited to only moderate consumption. As the main antioxidant components of a healthy diet, the following items were considered: selenium, vitamins $\mathrm{C}, \mathrm{A}$ and $\mathrm{E}$, tocopherol $\beta$, tocopherol $-\gamma$ and tocopherol $-\delta$, carotene- $\beta$ and carotene- $\alpha$, lycopene, lutein and all types of flavonoids.

Monounsaturated, saturated or polyunsaturated fatty acids and fibre intake from diet was expressed as $\mathrm{g} /$ day.

\section{Data collection}

Body mass index (BMI) was calculated as $\mathrm{kg} / \mathrm{m}^{2}$. Waist circumferences was measured according to the National Institute of Health, Heart, Lung and Blood guidelines. ${ }^{28}$ Blood pressure was measured by an automatic device (OMRON-HEM-705CP) three times on the non-dominant arm, with the patient lying down for about $5 \mathrm{~min}$. Hypertension was defined as systolic $\mathrm{BP} \geq 140 \mathrm{~mm} \mathrm{Hg}$ and/or diastolic $\mathrm{BP} \geq 90 \mathrm{~mm} \mathrm{Hg}$ or by the use of pharmacological treatment. ${ }^{29}$ Diabetes was defined if the glucose level was $\geq 126 \mathrm{mg} / \mathrm{dL}$ or by the use of pharmacological treatment. Hypercholesterolaemia status was defined if the total cholesterol level was $\geq 240 \mathrm{mg} / \mathrm{dL}$ or by the use of antihyperlipidemic treatment.

Physical activity was assessed by a structured questionnaire (24 questions on working and leisure time and sport participation) and expressed as daily energy expenditure in metabolic equivalent task-hours (MET/ day). Serum lipids and glucose were assayed by enzymatic reaction methods using an automatic analyser (ILab 350, Instrumentation Laboratory (IL), Milan, Italy). Low-density lipoprotein cholesterol was calculated according to Friedewald.

\section{Health-related quality of life and socioeconomic variables}

Health-related quality of life was assessed by using the validated Italian version of the self-administered SF-36. ${ }^{30}$ SF-36 is a general health scale that is widely used and thoroughly validated ${ }^{31}$ and has been translated into a number of languages, including Italian.

The questionnaire contains 36 items measuring eight multi-item parameters of health status covering the following domains: physical functioning, role limitations because of physical health problems, bodily pain, general health perceptions, vitality, social functioning, role limitations due to emotional problems and mental health. The first four domains deal with physical aspects and generate the physical component score, while the next four reflect psychological features and generate the mental component score.

For each domain, z-scores are calculated, summed and the sum transformed to a scale from 0 (the worst possible condition) to 100 (the best possible condition). ${ }^{32}$

In our study, the two scores expressed as continuous variables were then ranked into quartiles to allow specific statistical analyses.

Income categories were considered as low $(\leq 10000$ euros/year), low-medium (>10 000 $\leq 20000$ euros/year), medium-high $(>20000 \leq 40000$ euros/year) and high ( $>40000$ euros/year).

Socioeconomic status (SES) was expressed as a score based on five variables: dwelling ownership and ratio between the number of rooms and the number of livingin family members (rooms per person), currently and at childhood, and the availability of hot water at home at childhood. The five components were dichotomised according to the median value, and a score of one was attributed to the category supposed to be a marker of higher social status in comparison with the opposite category: thus, we assigned a score of 1 to people living in a house with living-in family members/room density $>0.6$, dwelling ownership or with availability of hot water and a score of 0 to people with living-in family members/room density $\leq 0.6$, no dwelling ownership or with unavailability of hot water. The total SES score did not include either income or education.

Education level was divided into two categories: $\leq 8$ years of studies ( 0 point) and $>8$ years of studies $(1$ point).

\section{STATISTICS}

Values for continuous variables are means \pm standard deviation (SD). Analysis of variance for continuous or categorical variables was applied to test the associations in table 1. Multivariable linear regression analysis was 
used for testing the association between mental and physical health scores (considered as continuous dependent variables) with adherence to MDS or dietary patterns (considered as continuous independent variables); linear regression coefficients were multiplied by $\mathrm{SD}$ of the independent variable. In this way, regression coefficients represent the variation in mental or physical scores for a $1 \mathrm{SD}$ change in independent variables, thus allowing comparability of the strength of the association among MDS, IMI or dietary patterns with mental and physical health.

By using multivariable logistic regression analysis, odds ratios (ORs) with a corresponding $95 \%$ confidence intervals (CI) were calculated to quantify the association of mental and physical health scores with adherence to dietary patterns. Four categories of adherence to MD were considered, ranging from low (0-2 points for MDS and IMI) to high ( $>6$ points for MDS and $\geq 5$ points for IMI) adherence. Dietary patterns from PCA were ranked into quartiles. For logistic regression analysis, the first quartiles of mental $(n=4234)$ or physical health were opposed to the fourth quartile $(n=4234)$. Participants with intermediate values (second and third quartiles) were excluded from this analysis to focus on the two extreme categories of self-reported health.

For linear and logistic regression analysis, potential confounders included as covariates in the models were age, sex, BMI, total energy intake, total physical activity, education, income, total socioeconomic status, smoking, diabetes, hypertension and hypercholesterolaemia. A specific category was created either for income or SES in order to account for missing values in the multivariable analyses.

Further adjustments for FAC, dietary fibre and fatty acids components were considered for testing their role in explaining the association among mental and physical health and MD markers.

The data analysis was generated using SAS/STAT software, V.9.1.3 of the SAS System for Windows 2009. SAS Institute Inc and SAS are registered trademarks of SAS Institute Inc, Cary, North Carolina, USA.

\section{RESULTS}

Table 1 shows the characteristics of the whole population according to the quartiles of mental and physical component scores. People in the uppermost quartile of selfreported mental health were $62.5 \%$ men and showed higher levels of leisure-time physical activity and a higher prevalence of high and medium-high income. Subjects in the highest quartile of physical health were younger, had lower BMI, as well as higher education, income and socioeconomic status. In addition, they had a lower prevalence of obesity, hypertension, diabetes and hypercholesterolaemia.

In table 2, the association between dietary scores and mental and physical health (considered as continuous variables) is reported.
Self-reported mental health was positively associated with greater adherence to MD in the fully adjusted model ( $\beta=0.33 ; 0.36 ; 0.50$ for MDS, IMI and olive oil and vegetables pattern, respectively) but inversely related to unhealthy dietary patterns $(\beta=-0.33$ for the eggs and sweets dietary pattern). The same positive association with MD was found for self-reported physical health, although the $\beta$-coefficients were lower than those observed for mental health ( $\beta=0.15 ; 0.08 ; 0.15$ for MDS, IMI and olive oil and vegetables pattern). Conversely, physical health was negatively associated with the meat and pasta dietary pattern $(\beta=-0.11)$.

Multivariable logistic regression analysis showed that the odds of being in the uppermost level of mental health clearly increased according to the categories of adherence to the Mediterranean-like eating patterns (table 3). People in the highest category of adherence had either $42 \%$ (MDS) or $34 \%$ (IMI) or $59 \%$ (olive oil and vegetables pattern) statistically significant higher probabilities to be in the uppermost category of mental health than those in the lowest group of MD adherence (table 3).

Logistic regression analysis showed different results regarding physical health (table 4). Higher MD adherence was not associated with increased odds of being in the highest physical health category. Modest results were found only for the olive oil and vegetable pattern for which ORs of $22 \%$ for the highest category of adherence were detected ( $p$ value $=0.01$ ). However, physical health was inversely correlated with adherence to the meat and pasta pattern, (table 4) but positively correlated with the eggs and sweets pattern; in the latter case, people in the low-medium or highest quartile had $24 \%$ more chances to be in the top category of the physical component score (table 4).

MDS was positively associated with all four domains of mental health $(\beta=0.49$ and standard error $(\mathrm{SE}) \pm 0.06$; $\beta=0.25$ and $\mathrm{SE} \pm 0.06 ; \beta=0.16$ and $\mathrm{SE} \pm 0.06 ; \beta=0.38$ and $\mathrm{SE}$ \pm 0.07 ; for vitality, social functioning, role limitation and mental health, respectively, $\mathrm{p}<0.05$ for all) and with three out of four domains of physical health $(\beta=0.34$ and $\mathrm{SE} \pm 0.06 ; \beta=0.18$ and $\mathrm{SE} \pm 0.06 ; \beta=0.16$ and $\mathrm{SE} \pm 0.05$; for physical functioning, role limitation due to physical functioning and general health, respectively, $\mathrm{p}<0.05$ for all), whereas no association was found for bodily pain $(\beta=-0.01$ and $\mathrm{SE} \pm 0.03 ; \mathrm{p}=0.73)$.

All the associations described above remained unchanged after further exclusion of subjects with diabetes $(n=1240 ; p<0.003$ for all $)$.

\section{Dietary antioxidant, fibre intake and fatty acids content}

In this study, we also tested the possible accounting of key dietary components of MD for the association between dietary scores and mental or physical health.

The FAC score was positively associated with MDS $(\beta=0.27, \mathrm{SE} \pm 0.01 ; \mathrm{P}$ for trend $<0.0001 ; \beta=0.36, \mathrm{SE} \pm 0.01 ; \mathrm{P}$ for trend $<0.0001 ; \beta=0.20, \mathrm{SE} \pm 0.003$; $\mathrm{P}$ for trend $<0.0001$ for MDS, IMI and olive oil and vegetables pattern, 


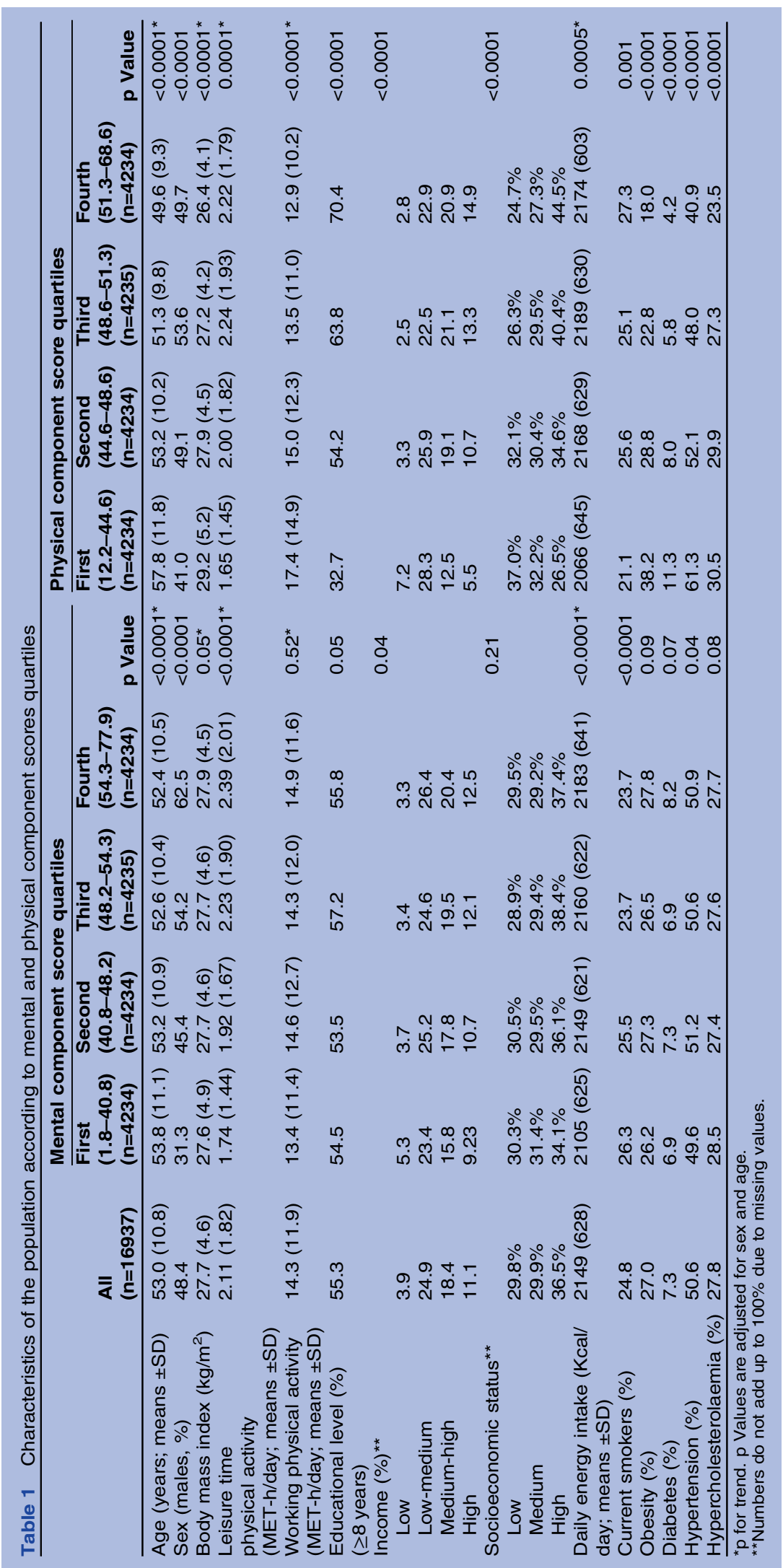

Bonaccio M, Di Castelnuovo A, Bonanni A, et al. BMJ Open 2013;3:e003003. doi:10.1136/bmjopen-2013-003003 


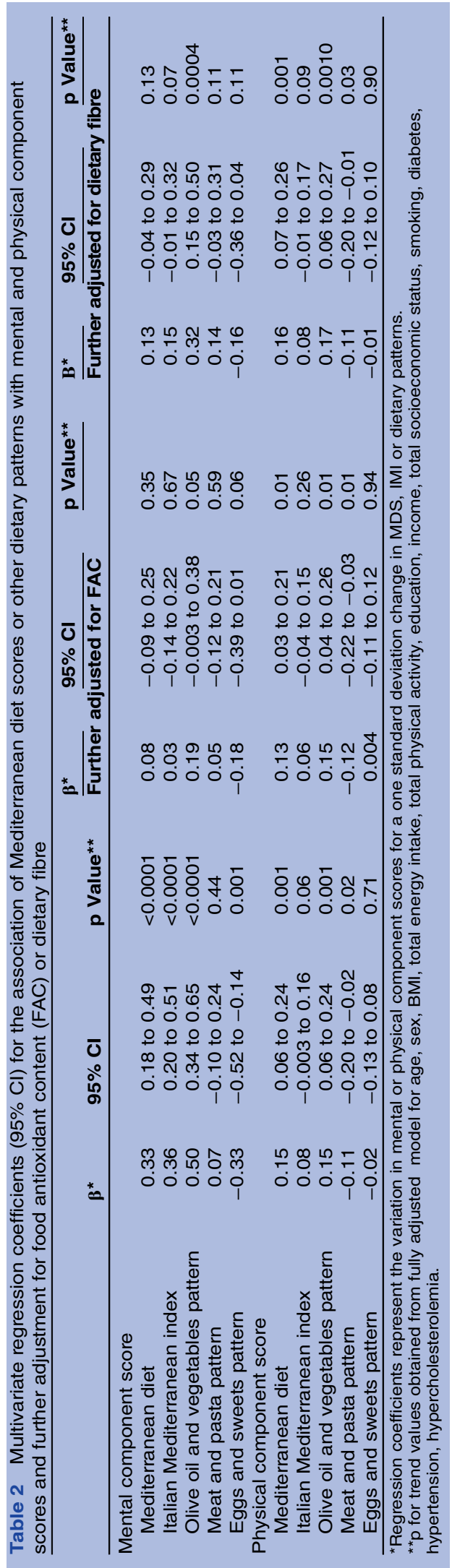

respectively) but inversely associated with the eggs and sweets pattern $(\beta=-0.04, \mathrm{SE} \pm 0.04$; $\mathrm{P}$ for trend $<0.0001)$, while no significant relationship was observed with the meat and pasta pattern $(\beta=0.005, \mathrm{SE} \pm 0.003$; $\mathrm{P}$ for trend $=$ $0.16)$. The FAC score was significantly related to mental health $(\beta=0.25, \mathrm{SE} \pm 0.04 ; \mathrm{P}$ for trend $<0.0001)$ but not to physical health $(\beta=0.002, \mathrm{SE} \pm 0.02$; $\mathrm{P}$ for trend $=0.92)$.

The inclusion of the FAC score in the multivariable models abolished the association between MD and mental health (table 2). Similar results were obtained when the FAC score was included in the logistic regression analysis model (table 3 ).

Monounsaturated fatty acids were positively associated with mental health $(\beta=0.04, \mathrm{SE} \pm 0.01$; $\mathrm{P}$ for trend= $0.0004)$ but not with physical health $(\beta=0.01, \mathrm{SE} \pm 0.01$; $\mathrm{P}$ for trend=0.06).

Polyunsaturated fatty acids were positively associated with MDS $(\beta=0.10 \mathrm{SE} \pm 0.01 ; p$ for trend $<0.0001 ; \beta=0.20$, $\mathrm{SE} \pm 0.01 ; \mathrm{p}$ for trend $<0.0001 ; \beta=0.22, \mathrm{SE} \pm 0.004 ; \mathrm{p}$ for trend $<0.0001$ for MDS, IMI and olive oil and vegetables pattern, respectively) and the eggs and sweets pattern $(\beta=0.19, \mathrm{SE} \pm 0.003$; $p$ for trend $<0.0001)$ but inversely linked to the meat and pasta pattern $(\beta=-0.07, \mathrm{SE} \pm 0.004$; $\mathrm{p}$ for trend $<0.0001)$. No relationship was found between polyunsaturated fatty acids and mental $(\beta=0.03, \mathrm{SE} \pm 0.05$; $\mathrm{P}$ for trend $=0.52)$ or physical health $(\beta=-0.05, \mathrm{SE} \pm 0.03 ; \mathrm{P}$ for trend $=0.08$ ).

Saturated fatty acids were positively associated with physical health $(\beta=0.02, \mathrm{SE} \pm 0.01$; $\mathrm{P}$ for trend=0.006) but not with mental health $(\beta=-0.01, \mathrm{SE} \pm 0.01 ; \mathrm{P}$ for trend $=0.21)$.

The inclusion of dietary monounsaturated or saturated fatty acids in the multivariable model did not modify the association between MD and mental $(p<0.0001)$ or physical health $(p<0.05$ for all; for the IMI adjusted for monounsaturated fatty acids $p$ value $=0.17$ ) (see online supplementary table). Similarly, no changes were recorded after including dietary polyunsaturated fatty acids in the multivariate models $(p<0.05$ for both mental and physical health) (see online supplementary table).

Dietary fibre consumption was positively associated with MDS $(\beta=0.15, \mathrm{SE} \pm 0.002)$, IMI $(\beta=0.16, \mathrm{SE} \pm 0.002)$ and the olive oil and vegetable pattern $(\beta=0.08$, SE \pm 0.001 ; $p$ values $<0.0001$ for all) but inversely associated with the meat and pasta $(\beta=-0.02, \mathrm{SE} \pm 0.001 ; \mathrm{p}<0.0001)$ and the eggs and sweets patterns $(\beta=-0.04, \mathrm{SE} \pm 0.001$; $\mathrm{p}<0.0001)$. Fibre content was also positively associated with either mental health or less consistently with the physical component score $(\beta=0.11, \mathrm{SE} \pm 0.01, \mathrm{p}<0.0001$; $\beta=0.02, \mathrm{SE} \pm 0.01, \mathrm{p}=0.01$ respectively).

Once the fibre content from the diet was included in the fully adjusted model, the association between the Mediterranean-like eating patterns and mental health was clearly reduced for both MDS $(\beta=0.33, p<0.0001$ decreased to $\beta=0.13, p=0.13)$ and IMI $(\beta=0.36, p<0.0001$ decreased to $\beta=0.15, p=0.07$ ), suggesting indeed a role of dietary fibres for the explanation of the observed association between either MDS or IMI and better mental 


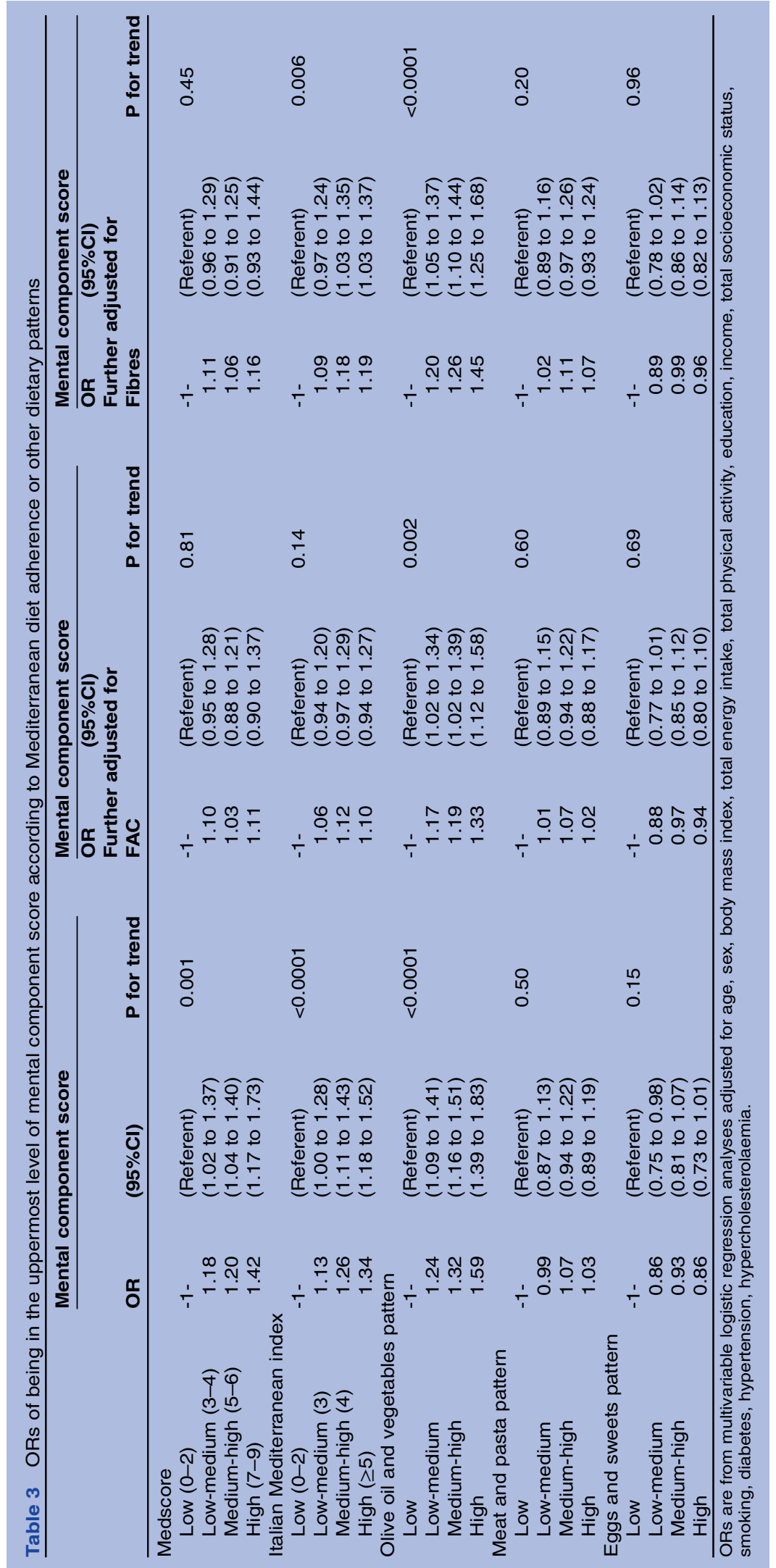

Bonaccio M, Di Castelnuovo A, Bonanni A, et al. BMJ Open 2013;3:e003003. doi:10.1136/bmjopen-2013-003003 


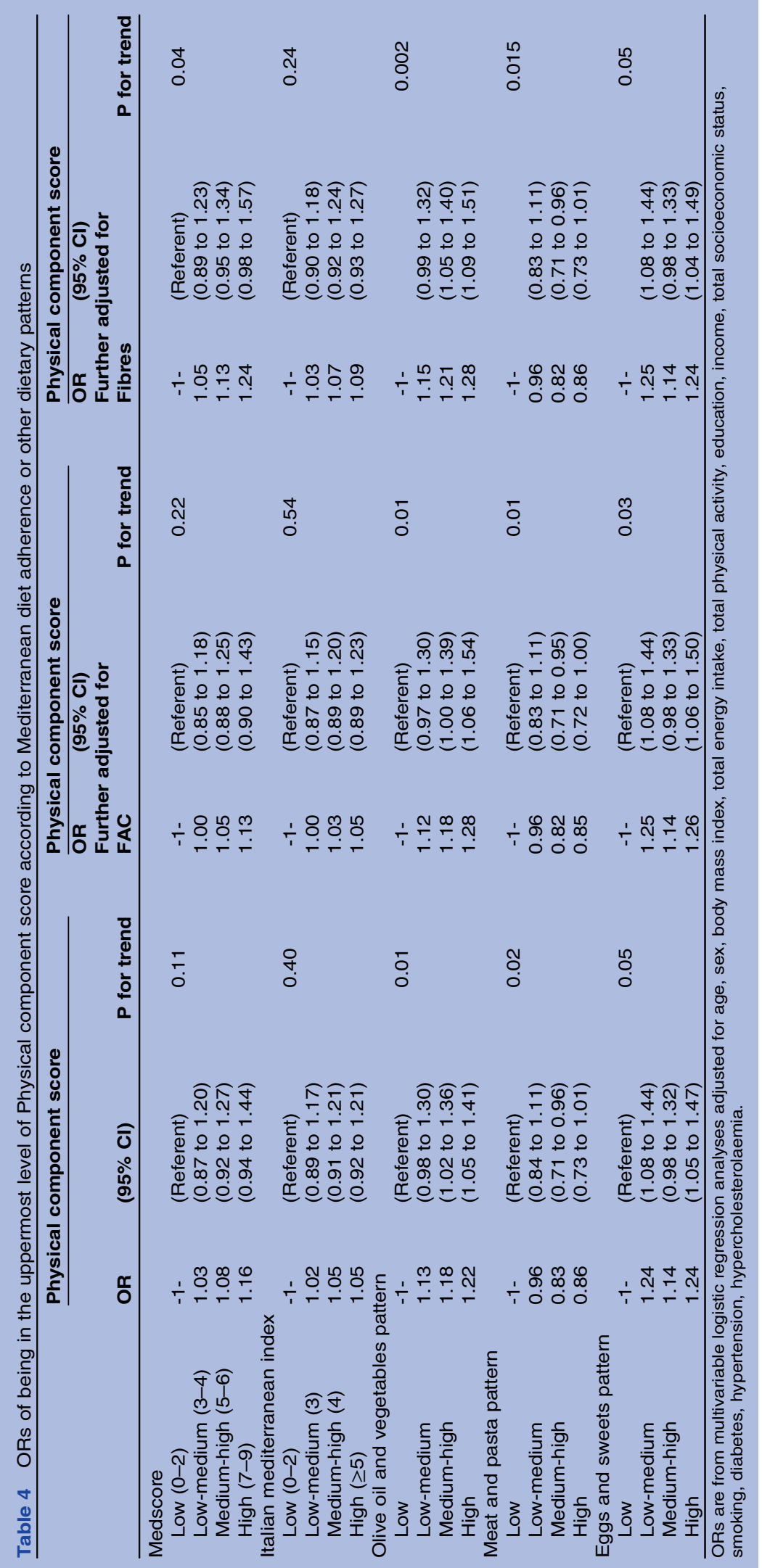


health (table 2). No significant changes were detected regarding the physical component score after adjustment for dietary fibre (table 2). The logistic regression analysis provided similar results (tables 3 and 4 ).

We performed further analysis by gender (data not shown) but failed to find any statistically significant sexrelated difference in the association of mental or physical scores with MDS or other dietary patterns as measured by specific terms of interaction (all $\mathrm{p}>0.05$ ).

\section{DISCUSSION}

In this large sample of a general adult population from the Moli-sani study, health-related quality of life was associated with high adherence to Mediterranean-like eating patterns and inversely related to unhealthy dietary habits. Our data agree with previous findings within Mediterranean cohorts, ${ }^{13} 14$ although in our study the relationship with MD was more consistent for mental health than for physical health.

Adherence to an MD pattern has been positively associated with reduced risk for a wide range of chronic diseases, including neurodegenerative disorders ${ }^{1}$ and cognitive decline, ${ }^{33} 34$ which are closely linked to selfperceived mental health. ${ }^{35} 36$ The beneficial effects of MD have been ascribed to its essential components. Antioxidants and polyphenols, largely present in Mediterranean foods, such as plant foods, fruits, olive oil and red wine, have anti-inflammatory properties and have been proven to exert a positive role against cardiovascular diseases and cancer. ${ }^{57}$ Also, monounsaturated fatty acids intake, whose major source is represented by olive oil, was found to be associated with a reduced prevalence of risk factors for major chronic disease, ${ }^{38} 39$ while a higher consumption of dietary fibre has beneficial effects on CVD risk reduction and is linked to a lower risk of fatal ischaemic heart disease. ${ }^{18}{ }^{19}$ In the wake of this evidence, we sought to examine whether the positive relationship between higher adherence to MD patterns and highest HRQL could be accounted for by the total antioxidant content from diet or by other compounds such as monounsaturated or polyunsaturated fatty acids or dietary fibre.

Higher intake of dietary antioxidants accounted for the positive association between MD adherence and higher mental status. In contrast, the levels of dietary antioxidants were not associated with physical health and thus failed to explain the observed relationship between higher adherence to MD and better self-reported physical status. Dietary fibre too was positively linked to improved mental health and apparently contributed to the explanation of the positive association with $\mathrm{MD}$, however in a less consistent way as compared to the antioxidant intake. As observed with the antioxidant content, dietary fibre did not account for the association between physical health and higher adherence to MD.

Conversely, neither monounsaturated nor polyunsaturated fatty acids from the diet modified the association between MD and better quality of life. However, data on polyunsaturated fatty acids should be taken with precaution since we could not distinguish the two major classes of polyunsaturated fatty acids (omega-3 or omega-6), which have opposing biological activities.

Previous studies have investigated the role of the basic components of MD on quality of life finding direct associations between some of its components and HRQL, such as fish consumption with mental health ${ }^{40}$ or with self-reported physical health. ${ }^{41}$ Inverse associations were also found between fruit and vegetables consumption and incidence of depressive symptoms. ${ }^{42}$ Yet this kind of approach fails to consider diet as a whole, most likely ending up with underestimating the interactions among several foods and nutrients. ${ }^{43}$ In addition to using a more traditional tool to evaluate adherence to healthy habits by using two a priori scores, our study approached overall diet as a combination of healthy and less healthy foods with an a posteriori approach which is able to catch the real dietary habits of people and accounts for correlations between score components.

Although previous studies had provided interesting data on the direct association between MD and better HRQL,${ }^{14}$ so far they have not approached the issue of possible mediators that are able to explain the observed beneficial effects of MD on self-reported health status.

Positive associations between antioxidants intake and cognitive function have been largely documented ${ }^{44} 45$ and were mainly ascribed to the preventing and retarding activity of antioxidants on the uncontrolled production of free radicals that might yield beneficial effects on the frontal/subcortical brain systems, with likely enhancement of cognitive functions. ${ }^{34}$ Oxidative stress has been implicated in the pathophysiology of many neuropsychiatric disorders ${ }^{46}$ such as schizophrenia, bipolar disorder ${ }^{47}$ and major depression. ${ }^{48}$

Recent epidemiological studies have confirmed a correlation between diet and depression, pointing out the importance of a diet rich in antioxidants and other essential compounds typical of MD in reducing the risk of depression, which may explain the positive effects in improving mental health. ${ }^{49}$

Regarding physical health, there are several biological and physiological mechanisms that could explain the beneficial effects of an MD pattern, such as reduced vascular inflammation and coagulation markers or improved endothelial function, ${ }^{50} 51$ all factors that are likely to be related to a better physical health status. However, in our study, neither the antioxidant content of diet nor the dietary fibre intake affected the association between Mediterranean scores and physical health. Improved physical health was also associated with higher adherence to the eggs and sweets pattern, characterised by high positive loadings of eggs, margarines, processed meat and sugar and sweets. Despite being rich in less healthy foods, this pattern has a high content of polyunsaturated fatty acids that could explain the positive relationship with improved physical health, as 
suggested by previous studies. ${ }^{40}$ However, the inclusion of polyunsaturated fatty acids in the model did not change this association. In the absence of any valid food compound that is able to explain the association between physical health and the eggs and sweets pattern, we cannot exclude a possible reverse-causality effect. Indeed, this eating pattern is mainly adopted by young people who are more confident about their physical health and thus could be more likely to disregard healthy dietary patterns. Although the data were age adjusted, a possible residual confounding due to age and other unmeasured age-related conditions cannot be excluded.

\section{Strengths of this study}

This work represents one of the few studies investigating the relationship between dietary patterns and health-related quality of life in a Mediterranean country. An additional strength is the large sample used for testing the association. In addition, it is apparently the first study to provide a likely account of antioxidants from the diet for the direct association between MD and HRQL to address this topic by using simultaneously two a priori Mediterranean scores and the a posteriori dietary patterns obtained by principal component analysis.

\section{Limitations of this study}

The present study was cross-sectional and shares all the limitations of this study type; in particular, inference of possible causality is unwarranted since it is not possible to determine whether the diet promotes well-being or whether those who 'feel better' choose to eat a healthy diet. However, in order to account for this reverse causality bias, we excluded from the analysis subjects with previous cancer, cardiovascular disease or diabetes as these conditions may have led to changes in their diet. We measured the overall intake of antioxidants by using a score based on estimates of micronutrient intake from the food frequency questionnaire. We recognise that this approach is challenging and that our indirect antioxidant intake assessment could have lower accuracy than direct measurements, which conversely are more difficult to perform in a very large sample size population.

Caution is needed in extending the results presented here to larger contexts since the data were collected in a region located between central and southern Italy, Mediterranean by tradition and culture. ${ }^{17}$ Yet, the main characteristics of our sample are comparable to those of the Italian Cardiovascular Epidemiological Observatory, ${ }^{52}$ a large survey including random samples of the general population all over Italy; therefore, our sample could be considered to be representative at least of the Italian population.

\section{CONCLUSIONS}

Our data indicate a positive association between adherence to the Mediterranean diet and self-reported mental and physical health status. The association is more obvious for mental than for physical health.

This large cross-sectional study has offered interesting indications on this issue by highlighting a major role played by dietary antioxidants in explaining the relationship between MD and HRQL. This is important in order to advance the hypothesis on the cause-effect relationship between MD and HRQL and to plan new strategies for the prevention of mental health decline in the general population.

\section{Author affiliations}

${ }^{1}$ Laboratory of Genetic and Environmental Epidemiology, Laboratori di

Ricerca, Fondazione di Ricerca e Cura "Giovanni Paolo II", Università Cattolica del Sacro Cuore, Campobasso, Italy

${ }^{2}$ Department of Epidemiology and Prevention, IRCCS Istituto Neurologico

Mediterraneo Neuromed, Pozzilli, Isernia, Italy

${ }^{3}$ Epicomed Research Srl, Campobasso, Italy

${ }^{4}$ Laboratory of Cardiovascular and Neurovascular Epidemiology, Casa di Cura Montevergine, Mercogliano, Avellino, Italy

Contributors $M B, A D C$ and $L I$ designed the research; FDL, MB, SC and FZ managed the data collection; $M B$ and $A D C$ analysed the data; $M B$ and $A B$ wrote the paper; GP developed the FAC score; MBD, GdG and LI originally inspired the research, obtained the financial support and critically reviewed the manuscript. All authors have read and approved the final version of the manuscript.

Funding The enrolment phase of the Moli-sani Project was supported by research grants from the Pfizer Foundation (Rome, Italy), Instrumentation Laboratory (IL, Milan, Italy) and the Italian Ministry of University and Research (MIUR, Rome, Italy)-Programma Triennale di Ricerca, Decreto no.1588. The Pfizer Foundation, IL and MIUR had no role in the study design, collection, analysis, and interpretation of data; in the writing of the report; and in the decision to submit the article for publication.

Competing interests None

Ethics approval The Moli-sani study was approved by the Ethics Committee of the Catholic University of Rome. Participants signed the informed consent before taking part in the study.

Provenance and peer review Not commissioned; externally peer reviewed. Data sharing statement No additional data are available.

Open Access This is an Open Access article distributed in accordance with the Creative Commons Attribution Non Commercial (CC BY-NC 3.0) license, which permits others to distribute, remix, adapt, build upon this work noncommercially, and license their derivative works on different terms, provided the original work is properly cited and the use is non-commercial. See: http:// creativecommons.org/licenses/by-nc/3.0/

\section{REFERENCES}

1. Sofi F, Abbate R, Gensini GF, et al. Accruing evidence on benefits of adherence to the Mediterranean diet on health: an updated systematic review and meta-analysis. Am J Clin Nutr 2010;92:1189-96.

2. Trichopoulou A, Bamia C, Trichopoulos D. Anatomy of health effects of Mediterranean diet: Greek EPIC prospective cohort study. BMJ 2009;338:b2337.

3. Bonaccio M, lacoviello L, de Gaetano G, On Behalf Of The Moli-Sani Investigators. The Mediterranean diet: the reasons for a Moli-Sani Investigators. The Mediterranean

4. Trichopoulou A, Vasilopoulou E, Lagiou A. Mediterranean diet and coronary heart disease: are antioxidants critical? Nutr Rev 1999;57:253-5.

5. Giacosa A, Barale R, Bavaresco L, et al. Cancer prevention in Europe: the Mediterranean diet as a protective choice. Eur $J$ Cancer Prev 2013;22:90-5.

6. Mattioli AV, Miloro C, Pennella S, et al. Adherence to Mediterranean diet and intake of antioxidants influence spontaneous conversion of atrial fibrillation. Nutr Metab Cardiovasc Dis 2013;23:115-21. 
7. Karr JE, Alexander JE, Winningham RG. Omega-3 polyunsaturated fatty acids and cognition throughout the lifespan: a review. Nutr Neurosci 2011;14:216-25.

8. Baumann C, Erpelding ML, Perret-Guillaume C, et al. Health-related quality of life in French adolescents and adults: norms for the DUKE Health Profile. BMC Public Health 2011;11:401.

9. Bardage C, Isacson D, Pedersen NL. Self-rated health as a predictor of mortality among persons with cardiovascular disease in Sweden. Scand J Public Health 2001:29:13-22.

10. Idler EL, Benyamini Y. Self-rated health and mortality: a review of twenty-seven community studies. J Health Soc Behav 1997;38:21-7.

11. Heidrich J, Liese AD, Löwel $\mathrm{H}$, et al. Self-rated health and its relation to all-cause and cardiovascular mortality in southern Germany. Results from the MONICA Augsburg cohort study 1984-1995. Ann Epidemiol 2002;12:338-45.

12. Nery Guimarães JM, Chor D, Werneck GL, et al. Association between self-rated health and mortality: 10 years follow-up to the Pró-Saúde cohort study. BMC Public Health 2012;12:676.

13. Sánchez PH, Ruano C, de Irala J, et al. Adherence to the Mediterranean diet and quality of life in the SUN Project. Eur $J$ Clin Nutr 2012;66:360-8.

14. Muñoz MA, Fíto M, Marrugat $J$, et al. Adherence to the Mediterranean diet is associated with better mental and physical health. Br J Nutr 2009;101:1821-7.

15. Costarelli V, Koretsi E, Georgitsogianni E. Health-related quality of life of Greek adolescents: the role of the Mediterranean diet. Qual Life Res 2013;22:951-6.

16. Sánchez-Villegas A, Delgado-Rodríguez M, Alonso A, et al. Association of the Mediterranean dietary pattern with the incidence of depression: the Seguimiento Universidad de Navarra/University of Navarra follow-up (SUN) cohort. Arch Gen Psychiatry 2009;66:1090-8

17. Rienks J, Dobson AJ, Mishra GD. Mediterranean dietary pattern and prevalence and incidence of depressive symptoms in mid-aged women: results from a large community-based prospective study. Eur J Clin Nutr 2013;67:75-8.

18. Satija A, Hu FB. Cardiovascular benefits of dietary fiber. Curr Atheroscler Rep 2012:14:505-14.

19. Crowe FL, Key TJ, Appleby PN, et al. Dietary fibre intake and ischaemic heart disease mortality: the European Prospective Investigation into Cancer and Nutrition-Heart study. Eur J Clin Nutr 2012;66:950-6.

20. Ajala O, English P, Pinkney J. Systematic review and meta-analysis of different dietary approaches to the management of type 2 diabetes. Am J Clin Nutr 2013:97:505-16.

21. Bonaccio M, Bonanni AE, Di Castelnuovo A, et al. Low income is associated with poor adherence to a Mediterranean diet and a higher prevalence of obesity: cross-sectional results from the Moli-sani study. BMJ Open 2012;2:e001685. doi:10.1136/bmjopen2012-001685

22. Pala V, Sieri S, Palli D, et al. Diet in the Italian EPIC cohorts: presentation of data and methodological issues. Tumori 2003;89:594-607.

23. Pisani P, Faggiano F, Krogh V, et al. Relative validity and reproducibility of a food frequency dietary questionnaire for use in the Italian EPIC centres. Int J Epidemiol 1997;26(Suppl 1):S152-60.

24. Centritto F, lacoviello L, di Giuseppe R, et al. Dietary patterns, cardiovascular risk factors and C-reactive protein in a healthy Italian population. Nutr Metab Cardiovasc Dis 2009;19:697-706.

25. Trichopoulou A, Costacou T, Bamia C, et al. Adherence to a Mediterranean diet and survival in a Greek population. $N$ Engl J Med 2003;348:2599-60819.

26. Agnoli C, Krogh V, Grioni S, et al. A priori-defined dietary patterns are associated with reduced risk of stroke in a large Italian cohort. J Nutr 2011;141:1552-8.

27. Pounis G, Costanzo S, di Giuseppe R, et al. Consumption of healthy foods at different content of antioxidant vitamins and phytochemicals and metabolic risk factors for cardiovascular disease in men and women of the Moli-sani study. Eur J Clin Nutr 2013;67:207-13.

28. Janssen I, Katzmarzyk PT, Ross R. Body mass index, waist circumference, and health risk: evidence in support of current National Institutes of Health guidelines. Arch Intern Med 2002;162:2074-9.

29. Guidelines Committee. 2003 European Society of HypertensionEuropean Society of Cardiology guidelines for the management of arterial hypertension. J Hypertens 2003;21:1011-53.
30. Apolone G, Mosconi P. The Italian SF-36 Health Survey: translation, validation and norming. J Clin Epidemiol 1998;51:1025-36.

31. Ware JE Jr, Gandek B. Overview of the SF-36 Health Survey and the International Quality of Life Assessment (IQOLA) Project. J Clin Epidemiol 1998;51:903-12.

32. Ware J, Sherbourne CD. The MOS 36-item short form health survey (SF-36). 1. Conceptual frame-work and item selection. Med Care 1992:30:473-81.

33. Naqvi AZ, Harty B, Mukamal KJ, et al. Monounsaturated, trans, and saturated fatty acids and cognitive decline in women. J Am Geriatr Soc 2011;59:837-43.

34. Panza F, Solfrizzi V, Colacicco AM, et al. Mediterranean diet and cognitive decline. Public Health Nutr 2004;7:959-63.

35. Visser M, Verbaan D, van Rooden S, et al. A longitudinal evaluation of health-related quality of life of patients with Parkinson's disease. Value Health 2009;12:392-6.

36. Karlsen $\mathrm{KH}$, Tandberg E, Arsland D, et al. Health related quality of life in Parkinson's disease: a prospective longitudinal study. J Neurol Neurosurg Psychiatry 2000;69:584-9.

37. Scoditti E, Calabriso N, Massaro M, et al. Mediterranean diet polyphenols reduce inflammatory angiogenesis through MMP-9 and COX-2 inhibition in human vascular endothelial cells: a potentially protective mechanism in atherosclerotic vascular disease and cancer. Arch Biochem Biophys 2012;527:81-9.

38. Brehm BJ, Lattin BL, Summer SS, et al. One-year comparison of a high-monounsaturated fat diet with a high-carbohydrate diet in type 2 diabetes. Diabetes Care 2009;32:215-20.

39. Baum SJ, Kris-Etherton PM, Willett WC, et al. Fatty acids in cardiovascular health and disease: a comprehensive update. J Clin Lipidol 2012;6:216-34.

40. Silvers KM, Scott KM. Fish consumption and self-reported physical and mental health status. Public Health Nutr 2002;5:427-31.

41. Schiepers OJ, de Groot RH, Jolles J, et al. Fish consumption, not fatty acid status, is related to quality of life in a healthy population. Prostaglandins Leukot Essent Fatty Acids 2010;83:31-5.

42. Liu C, Xie B, Chou CP, et al. Perceived stress, depression and food consumption frequency in the college students of China Seven Cities. Physiol Behav 2007;92:748-54.

43. Hu FB. Dietary pattern analysis: a new direction in nutritional epidemiology. Curr Opin Lipidol 2002;13:3-9.

44. Jama JW, Launer LJ, Witteman JC, Dietary antioxidants and cognitive function in a population-based sample of older persons. The Rotterdam study. Am J Epidemiol 1996;144: $275-80$.

45. Valls-Pedret C, Lamuela-Raventós RM, Medina-Remón A, et al. Polyphenol-rich foods in the Mediterranean diet are associated with better cognitive function in elderly subjects at high cardiovascular risk. J Alzheimers Dis 2012;29:773-82.

46. $\mathrm{Ng} \mathrm{F}$, Berk M, Dean O, et al. Oxidative stress in psychiatric disorders: evidence base and therapeutic implications. Int $J$ Neuropsychopharmacol 2008;11:851-76.

47. Andreazza AC, Kauer-Sant'anna M, Frey BN, et al. Oxidative stress markers in bipolar disorder: a meta-analysis. J Affect Disord 2008:111:135-44.

48. Maes M, De Vos N, Pioli R, et al. Lower serum vitamin $\mathrm{E}$ concentrations in major depression. Another marker of lowered antioxidant defenses in that illness. J Affect Disord 2000;58 241-6.

49. Jacka FN, Mykletun A, Berk M. Moving towards a population health approach to the primary prevention of common mental disorders. BMC Med 2012;10:149.

50. Esposito K, Marfella R, Ciotola $M$, et al. Effect of a Mediterranean-style diet on endothelial dysfunction and markers of vascular inflammation in the metabolic syndrome: a randomized trial. JAMA 2004;292:1440-6.

51. Chrysohoou C, Panagiotakos DB, Pitsavos C, et al. Adherence to the Mediterranean diet attenuates inflammation and coagulation process in healthy adults: the ATTICA study. J Am Coll Cardiol 2004:44:152-8.

52. Giampaoli S, Rielli R, Dematté L, et al. The Italian observatory of cardiovascular risk: the CUORE project experience [abstract]. Circulation 2009;119:139. 
Chapter 4.

Mushroom consumption and dietary selenium intake in association with fasting glucose levels in a free-living Italian adult population: the Moli-sani study

Pounis G, Costanzo S, Persichillo M, de Curtis A, Sieri S, Vinceti M, Zito F, Di Castelnuovo AF, Donati MB, de Gaetano G, lacoviello L, on behalf of the Moli-sani Project Investigators. Mushroom consumption and dietary selenium intake in association with fasting glucose levels in a free-living Italian adult population: the Moli-sani study. Diabetes \& Metabolism 2013. pii: S1262-3636(13)00180-8. doi: 10.1016/j.diabet.2013.09.007 


\section{Available online at}

ScienceDirect

www.sciencedirect.com

\author{
Elsevier Masson France

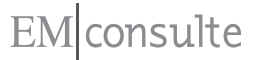 \\ www.em-consulte.com/en
}

Original article

\section{Mushroom and dietary selenium intakes in relation to fasting glucose levels in a free-living Italian adult population: The Moli-sani Project}

a Laboratory of Environmental and Genetic Epidemiology, Research Laboratories, Fondazione di Ricerca e Cura "Giovanni Paolo II", Università Cattolica, 86100 Campobasso, Italy

${ }^{\mathrm{b}}$ Department of Epidemiology and Prevention, IRCCS Istituto Neurologico Mediterraneo Neuromed, 86077 Pozzilli (Isernia), Italy ${ }^{\mathrm{c}}$ Epidemiology and Prevention Unit, Fondazione IRCCS Istituto Nazionale dei Tumori, Milan, Italy

${ }^{\mathrm{d}}$ Department of Diagnostic, Clinical and Public Health Medicine, University of Modena and Reggio Emilia, Modena, Italy

${ }^{\mathrm{e}}$ Laboratory of Cardiovascular and Neurovascular Epidemiology, Casa di Cura Montevergine, Mercogliano (AV), Italy Received 28 May 2013; received in revised form 20 September 2013; accepted 22 September 2013

\section{Abstract}

Aim. - Mushrooms are known to be a major food source of selenium, a mineral associated with diabetes prevalence. This study aimed to evaluate the relationship between mushroom and dietary selenium intakes and blood glucose levels in a free-living adult Italian population.

Methods. - A total of 6879 men and 6891 women (aged 53.1 \pm 11.0 years) with neither diabetes nor on special diets were analyzed from the population randomly recruited for the Moli-sani study. The European Prospective Investigation into Cancer and Nutrition (EPIC) food frequency questionnaire was used for dietary assessment. Fasting blood glucose (FBG) levels were measured from fasting serum samples, and diabetes prevalence was determined according to American Diabetes Association diagnostic criteria.

Results. - In men, an increase of one (30-g) portion/week in mushroom consumption was associated with a $0.43-0.55 \mathrm{mmol} / \mathrm{L}$ increase in FBG at different levels of adjustment $(P<0.05)$. In both men and women, dietary selenium was positively associated with blood glucose in both unadjusted and fully adjusted models $(P<0.05$ for all). Both mushroom and dietary selenium intakes were independently associated with blood glucose on multivariate analyses. In addition, high intakes of both were associated with higher diabetes prevalence in men and women $(\mathrm{OR}>1, P<0.05)$.

Conclusion. - The association of mushroom and selenium intakes with FBG suggests that mushroom and selenium might each independently increase the risk of diabetes. However, prospective studies are now necessary to confirm this hypothesis.

(c) 2013 Elsevier Masson SAS. All rights reserved.

Keywords: Mushroom; Selenium; Glucose; Diabetes; Dietary habits

\section{Résumé}

Consommation alimentaire de champignons et apport de sélénium chez des sujets adultes en Italie : résultats de l'étude Moli-sani.

Objectif. - Les champignons contribuent à l'apport de sélénium. Une hausse des niveaux plasmatiques de sélénium a été associée à la prévalence de diabète. Dans cette étude, nous avons évalué l'association de la consommation de champignons, et de l'apport de sélénium, avec la glycémie chez une population générale d'adultes italiens.

Méthodes. - Dans cette population, nous avons analysé 6879 hommes et 6891 femmes $(53,1+11,0$ ans) diabétiques ou qui suivaient des régimes spéciaux, par le questionnaire EPIC sur la fréquence de consommation des différent ingrédients. La glycémie a été mesurée à jeun et le diagnostique de diabète établi selon les critères de l'Association américaine de diabétologie.

Résultats. - Chez les hommes, une augmentation de l'apport de champignons (une portion par semaine, $30 \mathrm{~g}$ ) était associée avec une hausse de 0,43-0,55 mmol/L de glycémie avec ou sans ajustement. Le sélénium alimentaire était positivement associé avec la glycémie par des modèles avec ou sans ajustement $(P<0,05)$. L'apport de champignons et le niveau de sélénium étaient associés de façon indépendante avec la glycémie (analyse multivariée). Une augmentation des deux paramètres était associée avec une prévalence plus élevée de diabète.

* Corresponding author. Department of Epidemiology and Prevention, IRCCS Istituto Neurologico Mediterraneo Neuromed, 86077 Pozzilli (Isernia), Italy. Tel.: +390865929664 ; fax: +390865927575 .

E-mail address: licia.iacoviello@moli-sani.org (L. Iacoviello).

1262-3636/\$ - see front matter @ 2013 Elsevier Masson SAS. All rights reserved.

http://dx.doi.org/10.1016/j.diabet.2013.09.007

Please cite this article in press as: Pounis G, et al. Mushroom and dietary selenium intakes in relation to fasting glucose levels in a free-living Italian adult population: The Moli-sani Project. Diabetes Metab (2013), http://dx.doi.org/10.1016/j.diabet.2013.09.007 
Conclusions. - L'association de l'apport alimentaire de champignons et de sélénium pourrait influencer de façon indépendante le risque de diabète.

(C) 2013 Elsevier Masson SAS. Tous droits réservés

Mots clés : Champignons ; Sélénium ; Glycémie ; Diabète ; Habitudes alimentaires

\section{Introduction}

According to Chang [1], mushrooms are macrofungi with distinctive fruiting bodies that can be hypogeous (underground) or epigeous (above ground), and large enough to be seen with the naked eye and picked up by hand. This definition is most often applied to those fungi (classed as Basidiomycota and Agaricomycetes) that have a stem (stipe), a cap (pileus) and gills (lamellae, sing. lamella) or pores on the underside of the cap. Nowadays, mushrooms have fans around the world because of their unique taste and the experience they offer when gathered in the wild. However, in the past, mushrooms were mainly consumed in Japan and China, and have been studied in those countries not only for the development of functional foods, but also for medicinal food extracts [2]. In particular, three kinds of carcinostatic polysaccharide drugs, such as immunopotentiators (biological response modifiers), have been developed in Japan [2]. Studies in animal models and human cells have defined some of the mechanisms related to oxidative stress by which mushroom extracts might be of benefit against disease [3-7]. However, there is only limited epidemiological or clinical evidence coming mainly from Eastern populations regarding the health benefits of their consumption [8-12].

Besides other micronutrients and their low-calorie composition, mushrooms are among the richest sources of trace elements, including heavy metals and the metalloid selenium [13,14]. Recent epidemiological studies have associated elevated blood levels of selenium with higher prevalence and incidence of diabetes [15-17], while evidence has also emerged of a positive association of selenium with serum lipid levels and other cardiometabolic disease biomarkers. In addition, clinical trials have indicated adverse effects with selenium supplementation in humans $[18,21]$.

The high selenium content of mushrooms, lack of epidemiological evidence of their association with fasting blood glucose (FBG) levels and limited results for the relationship between food sources of selenium and FBG levels have prompted the need to:

- evaluate long-term mushroom and dietary selenium intakes in a free-living adult Italian population;

- determine the relationship between such intakes and FBG levels.

\section{Subjects and methods}

\subsection{Study population}

The cohort for the Moli-sani Project was recruited from the Molise region of Italy using City Hall registries and a multistage sampling procedure as described elsewhere [22,23]. Between March 2005 and April 2010, 24,325 subjects were considered for the study. However, individuals who had incomplete medical or dietary questionnaires $(n=1479)$, or were not Caucasian or born in Italy $(n=332)$ were excluded from the analysis, as were those who reported a history of cardiovascular disease $(n=1719)$ or cancer $(n=889)$. In addition, participants diagnosed with diabetes $(n=1036)$ or using hypoglycaemic treatment $(n=823)$ and those following a low-carbohydrate or other specific diet $(n=6154)$ were also excluded, as these conditions can lead to changes in blood glucose levels and dietary intakes. Thus, 6879 male and 6891 female participants were finally analyzed. In this way, any possible errors induced by the cross-sectional nature of the present setting were minimized.

The Catholic University Ethics Committee approved the Moli-sani Project. All participants provided their written informed consent.

\subsection{Measurement and definition of risk factors}

Blood pressure $(\mathrm{mmHg})$ was measured by an automatic device (HEM-705CP, Omron Corp., Kyoto, Japan), three times on the non-dominant arm with the patient lying down for about $5 \mathrm{~min}$; the second and third measurements were averaged and used for the analysis. Weight and height were measured with the subjects wearing light underwear and no shoes, and their body mass index (BMI) calculated.

All blood samples were obtained from participants who had fasted overnight and had refrained from smoking for at least $6 \mathrm{~h}$. Serum lipids and blood glucose were assayed by enzymatic reaction methods using an automatic analyzer (ILab 350, Instrumentation Laboratory, Bedford, MA, USA). Lowdensity lipoprotein (LDL) cholesterol was calculated according to the Friedewald equation. Haemochromocytometric analysis was performed by cell count (COULTER HmX Hematology Analyzer, Beckman Coulter, Brea, CA, USA).

Diabetes was defined according to the American Diabetes Association (ADA) diagnostic criteria [24] (FBG levels $>6.9 \mathrm{mmol} / \mathrm{L}$ or use of specific antidiabetic medication). Participants with blood pressure levels $\geq 140 / 90 \mathrm{mmHg}$ or using antihypertensive medications were classified as hypertensive. Hypercholesterolaemia and hypertriglyceridaemia were defined as total serum cholesterol levels $>5.2 \mathrm{mmol} / \mathrm{L}$ and triglyceride levels $>2.3 \mathrm{mmol} / \mathrm{L}$, respectively, or the use of lipid-lowering agents. Obesity was defined as BMI $>29.9 \mathrm{~kg} / \mathrm{m}^{2}$.

Subjects were classified as non-smokers if they had smoked fewer than 100 cigarettes in their lifetime or had never smoked cigarettes; as ex-smokers if they had smoked cigarettes in the past, but had stopped smoking for at least 1 year; and as current smokers if they had reported having smoked at least 100 
cigarettes in their lifetime and still smoked or had quit smoking within the previous year [25]. Socioeconomic status was defined by a score ranging from 0 to 8 , based on eight variables: the higher the score, the higher the socioeconomic status [22]. Physical activity was assessed by a structured questionnaire and expressed as daily metabolic equivalent of task (MET) energy expenditure (h) [26].

\subsection{Dietary assessment}

The European Prospective Investigation into Cancer and Nutrition (EPIC) food frequency questionnaire (FFQ) specifically adapted for the Italian population and including 188 food items was used to determine the usual nutritional intakes consumed during the past year [27]. A computer programme for nutrient analysis of the FFQ (NAF) [28] was developed by the Epidemiology and Prevention Unit of the IRCCS Foundation, National Cancer Institute of Milan, to convert questionnaire dietary data into frequency of consumption, average daily quantity of food (g/week), and dietary selenium and energy (Kcal) intakes. For the present analysis, NAF data were linked to Italian food composition tables for epidemiological studies [29]. Mushroom as a food group included any kind of mushrooms consumed by the Italian population. Tertiles of mushroom consumption were constructed $(<14,14-28$ and $>28 \mathrm{~g} /$ week). In addition, major food sources of selenium such as grains, pasta, nuts, seeds, legumes, potatoes, poultry and fish were assessed $[13,14]$, and quartiles of selenium intake were calculated $(<50$, $50-60,61-75,>75 \mu \mathrm{g} /$ day). The US Institute of Medicine's recommended dietary allowance (RDA) of $50-55 \mu \mathrm{g} /$ day of selenium was considered safe for the present population [30], and total antioxidant consumption was evaluated using the food antioxidant content (FAC) score developed previously by the Moli-sani study group [14].

\subsection{Statistical analysis}

Normally distributed continuous variables were presented as means (standard deviation), skewed variables as medians (between the 1st and 3rd quartiles) and categorical variables as frequencies. The normality of continuous variables was tested graphically. Comparisons of continuous variables between two study groups were performed using the independent Student's $t$ test for normally distributed variables or log-transformed skewed data. Comparisons of continuous variables within more than two groups were carried out using the one-way analysis of variance (ANOVA) F test for normally distributed variables and the Kruskal-Wallis test for skewed data. Correlations between pairs of continuous variables were done using Pearson's r correlation coefficient. In the case of skewed data, log-transformed variables were used.

Unadjusted and multiple-adjusted linear-regression analyses for men and women separately were used to evaluate the relationship between mushroom intake and FBG levels. In particular, five levels of adjustment were defined. In each model the outcome was FBG levels and the independent factor was log-transformed mushroom consumption [log (g/week)]. In all cases: Model 1 was unadjusted; Model 2 was adjusted for age and energy intake; Model 3 was further adjusted for physical activity, smoking habits, social status and the presence of obesity, hypertension, hypercholesterolaemia and hypertriglyceridaemia; Model 4 was further adjusted for dietary selenium; and Model 5 was further adjusted for grains, pasta, nuts, seeds, legumes, potatoes, poultry and fish intakes and FAC. Additional regression analyses were conducted with FBG levels as outcome, and gender, mushroom intake and their interaction term as independent variables.

To evaluate the possible association of dietary selenium with FBG levels, similar unadjusted and multiple-adjusted linear models were used with the same sample settings. Unadjusted and multiple-adjusted (for age, physical activity, smoking habits, social status, systolic and diastolic blood pressure, lipid profile markers, BMI, energy intake, consumption of grains, pasta, nuts, seeds, legumes, mushroom, potatoes, poultry and fish, and FAC score) analyses were performed. In addition, a nutrient residual (energy-adjusted) model was used to confirm the possible association of selenium intake with glucose levels [31]. In particular, the residuals predicted by simple linear-regression, with dietary selenium as the dependent factor and energy intake as the independent factor, were used in place of dietary selenium in unadjusted and multiple-adjusted regression models.

For both mushroom and dietary selenium intakes, separate analyses were conducted to establish whether their association with glucose was linear. Models including squared or cubed terms of these factors were constructed.

Normality of residuals, homoscedasticity and multiple collinearity were evaluated by plotting standardized residuals against predicted values. Results were presented as $\beta$ coefficients ( $\beta$-coef) and their 95\% confidence intervals (CI) for increases in mushroom consumption of two 30-g portions per week.

Unadjusted and multiple-adjusted logistic-regression analyses for men and women separately were used to evaluate the relationship between mushroom or selenium intake and the likelihood of the presence of diabetes. In each model the outcome was the presence of diabetes, and the independent factor was tertiles of mushroom consumption or quartiles of selenium intake. Deviance residuals evaluated goodness of fit. Results were presented as odds ratios (ORs) with their corresponding 95\% CI.

All tested hypotheses were two-sided, and $P$ values $<0.05$ were considered statistically significant. STATA version 9 software was used for all calculations (StataCorp LP, College Station, TX, USA).

\section{Results}

After excluding diabetic participants and those on lowcarbohydrate or other specific diets, the analyses included the 6879 men and 6891 women comprising the total Moli-sani population. Median mushroom intakes in men and women were $14.0(4.9,28.0) \mathrm{g} /$ week and $14.0(3.5,28.0) \mathrm{g} /$ week, respectively ( $P=0.62$ by $t$ test on log-transformed mushroom intake). Tables 1 and 2 present the distribution of general characteristics by gender according to weekly mushroom consumption. Men in the highest tertile of mushroom consumption were younger, 


+Model
DIABET 5651-9

G. Pounis et al. / Diabetes \& Metabolism xxx (2013) xxx-xxx

Table 1

Characteristics of the male Moli-sani population $(n=6879)$ according to mushroom consumption.

\begin{tabular}{|c|c|c|c|c|}
\hline & \multicolumn{3}{|c|}{ Mushroom consumption } & \multirow[t]{2}{*}{$P$ value } \\
\hline & $<14 \mathrm{~g} /$ week & $14-28 \mathrm{~g} /$ week & $>28 \mathrm{~g} /$ week & \\
\hline \multicolumn{5}{|l|}{ Lifestyle characteristics } \\
\hline Age (> 55 years, $\%)$ & 45.1 & 40.3 & 35.5 & $<0.001$ \\
\hline Physically active (\%) & 51.6 & 51.1 & 52.6 & 0.63 \\
\hline Current smokers (\%) & 28.6 & 31.3 & 30.8 & 0.15 \\
\hline Social status $(0-6)$ & $3.48(1.39)$ & $3.51(1.34)$ & $3.65(1.35)$ & $<0.001$ \\
\hline \multicolumn{5}{|l|}{ Clinical characteristics } \\
\hline Fasting blood glucose $(\mathrm{mmol} / \mathrm{L})$ & $5.61(0.94)$ & $5.66(0.94)$ & $5.66(1.17)$ & 0.27 \\
\hline Hypertension (\%) & 59.2 & 53.7 & 50.1 & $<0.001$ \\
\hline Hypercholesterolaemia (\%) & 59.7 & 62.5 & 63.2 & 0.07 \\
\hline Hypertriglyceridaemia (\%) & 33.4 & 33.3 & 33.3 & 0.24 \\
\hline Obesity (\%) & 28.8 & 28.3 & 28.5 & 0.96 \\
\hline \multicolumn{5}{|l|}{ Dietary habits } \\
\hline Energy intake (Kcal/day) & $2298(1922,2725)$ & $2370(2010,2791)$ & $2424(2029,2890)$ & $<0.001$ \\
\hline Grains \& pasta (g/week) & $520(343,671)$ & $544(367,680)$ & $491(332,636)$ & 0.003 \\
\hline Nuts \& seeds (g/week) & $2.1(1.4,3.5)$ & $2.1(1.4,2.1)$ & $2.1(1.4,5.6)$ & 0.04 \\
\hline Legumes (g/week) & $94.5(51.8,129)$ & $85.4(50.4,123)$ & $101(58.1,135)$ & $<0.001$ \\
\hline Potatoes (g/week) & $165(88.9,322)$ & $166(93.1,326)$ & $174(151,331)$ & $<0.001$ \\
\hline Poultry (g/week) & $104(72.1,206)$ & $104(73.5,206)$ & $104(82.6,206)$ & $<0.001$ \\
\hline Fish (g/week) & $114(53.2,174)$ & $114(53.2,174)$ & $162(81.2,174)$ & $<0.001$ \\
\hline Dietary selenium ( $\mu \mathrm{g} /$ day $)$ & $59.6(50.0,71.5)$ & $62.9(52.6,75.2)$ & $67.4(55.7,81.2)$ & $<0.001$ \\
\hline Selenium intake $>55 \mu \mathrm{g} /$ day (RDA) & 61.4 & 69.4 & 76.4 & $<0.001$ \\
\hline FAC score $(-99,99)$ & $0(-37,39)$ & $4(-33,41)$ & $13(-27,50)$ & $<0.001$ \\
\hline
\end{tabular}

RDA: recommended dietary allowance; FAC: food antioxidant content. Normally distributed continuous variables are presented as means (SD), skewed variables as medians (between 1st and 3rd quartiles) and categorical variables as frequencies.

a Differences derived by Pearson's Chi-square test for categorical data, one-way ANOVA F tests for normally distributed variables and Kruskal-Wallis test for skewed variables.

Table 2

Characteristics of the female Moli-sani population $(n=6891)$ according to mushroom consumption.

\begin{tabular}{|c|c|c|c|c|}
\hline & \multicolumn{3}{|c|}{ Mushroom consumption } & \multirow[t]{2}{*}{$P$ value $^{\mathrm{a}}$} \\
\hline & $<14 \mathrm{~g} /$ week & $14-28 \mathrm{~g} /$ week & $>28 \mathrm{~g} /$ week & \\
\hline \multicolumn{5}{|l|}{ Lifestyle characteristics } \\
\hline Age $(>55$ years, $\%)$ & 40.3 & 33.3 & 31.2 & $<0.001$ \\
\hline Physically active (\%) & 50.0 & 48.3 & 54.6 & $<0.001$ \\
\hline Current smokers $(\%)$ & 20.3 & 22.7 & 24.8 & 0.006 \\
\hline Social status $(0-6)$ & $3.46(1.37)$ & $3.61(1.38)$ & $3.60(1.40)$ & 0.002 \\
\hline \multicolumn{5}{|l|}{ Clinical characteristics } \\
\hline Fasting blood glucose $(\mathrm{mmol} / \mathrm{L})$ & $5.16(0.78)$ & $5.16(0.72)$ & $5.16(0.72)$ & 0.21 \\
\hline Hypertension (\%) & 47.9 & 39.4 & 36.8 & $<0.001$ \\
\hline Hypercholesterolaemia (\%) & 64.9 & 63.5 & 61.7 & 0.14 \\
\hline Hypertriglyceridaemia (\%) & 17.5 & 17.1 & 15.0 & 0.10 \\
\hline Obesity (\%) & 28.2 & 25.2 & 25.9 & 0.11 \\
\hline \multicolumn{5}{|l|}{ Dietary habits } \\
\hline Energy intake (Kcal/day) & $1987(1696,2335)$ & $2044(1737,2388)$ & $1860(1556,2236)$ & $<0.001$ \\
\hline Grains \& pasta (g/week) & $378(249,550)$ & $396(267,550)$ & $380(246,548)$ & 0.05 \\
\hline Nuts \& seeds (g/week) & $2.1(1.4,2.1)$ & $2.1(1.4,2.1)$ & $2.1(1.4,3.5)$ & 0.17 \\
\hline Legumes (g/week) & $94.9(49.0,135)$ & $94.5(50.6,125)$ & $105(59.5,137)$ & $<0.001$ \\
\hline Potatoes (g/week) & $165(86.1,322)$ & $167(88.9,324)$ & $173(121,329)$ & $<0.001$ \\
\hline Poultry (g/week) & $104(72.8,206)$ & $104(74.2,206)$ & $104(88.2,206)$ & $<0.001$ \\
\hline Fish (g/week) & $114(53.2,174)$ & $114(53.2,174)$ & $122(79.8,174)$ & $<0.001$ \\
\hline Dietary selenium ( $\mu \mathrm{g} /$ day) & $54.9(45.8,66.8)$ & $58.2(48.3,70.3)$ & $61.1(50.6,73.6)$ & $<0.001$ \\
\hline Selenium intake $>55 \mu \mathrm{g} /$ day $(\mathrm{RDA}$ & 49.7 & 58.6 & 64.9 & $<0.001$ \\
\hline FAC score $(-99,99)$ & $-3(-43,35)$ & $-4(-40,33)$ & $8(-29,45)$ & $<0.001$ \\
\hline
\end{tabular}

RDA: recommended dietary allowance; FAC: food antioxidant content. Normally distributed continuous variables are presented as means (SD), skewed variables as medians (between 1st and 3rd quartiles) and categorical variables as frequencies.

a Differences derived by Pearson's chi-square test for categorical data, one-way ANOVA F tests for normally distributed variables and Kruskal-Wallis test for skewed variables. 
Table 3

Mushroom and dietary selenium intakes in relation to fasting blood glucose (FBG) levels in the Moli-sani population.

\begin{tabular}{|c|c|c|c|c|c|}
\hline & \multicolumn{5}{|c|}{ FBG levels (mmol/L), $\beta$-coef $(95 \% \mathrm{CI})$} \\
\hline & $\begin{array}{l}\text { Model } 1 \\
\text { (crude) }\end{array}$ & $\begin{array}{l}\text { Model } 2 \\
\text { (multiple-adjusted) }\end{array}$ & $\begin{array}{l}\text { Model } 3 \\
\text { (multiple-adjusted) }\end{array}$ & $\begin{array}{l}\text { Model } 4 \\
\text { (multiple-adjusted) }\end{array}$ & $\begin{array}{l}\text { Model } 5 \\
\text { (multiple-adjusted) }\end{array}$ \\
\hline \multicolumn{6}{|l|}{ Mushroom consumption } \\
\hline \multicolumn{6}{|l|}{$\operatorname{Men}(n=6879)$} \\
\hline $\begin{array}{c}\beta \text {-coef for } 1(30-\mathrm{g}) \\
\text { portion/week increase }\end{array}$ & $0.27(-0.12,0.65)$ & $0.43(0.04,0.82)$ & $0.55(0.19,0.91)$ & $0.46(0.09,0.82)$ & $0.47(0.11,0.83)$ \\
\hline Women $(n=6891)$ & & & & & \\
\hline $\begin{array}{c}\beta \text {-coef for } 1(30-\mathrm{g}) \\
\text { portion/week increase }\end{array}$ & $0.24(-0.54,0.08)$ & $0.02(-0.29,0.33)$ & $0.07(-0.23,0.33)$ & $0.09(-0.29,0.32)$ & $0.02(-0.29,0.32)$ \\
\hline \multicolumn{6}{|l|}{ Dietary selenium } \\
\hline \multicolumn{6}{|l|}{ Men $(n=6879)$} \\
\hline$\beta$-coef for $50 \mu \mathrm{g} /$ day increase & $0.10(0.04,0.16)$ & $0.16(0.08,0.24)$ & $0.10(0.03,0.17)$ & $-{ }^{\mathrm{a}}$ & $0.14(0.06,0.23)$ \\
\hline \multicolumn{6}{|l|}{ Women $(n=6891)$} \\
\hline$\beta$-coef for $50 \mu \mathrm{g} /$ day increase & $0.04(-0.01,0.09)$ & $0.13(0.07,0.19)$ & $0.08(0.03,0.14)$ & $-{ }^{\mathrm{a}}$ & $0.11(0.03,0.18)$ \\
\hline
\end{tabular}

CI: confidence intervals. This analysis excludes diabetics and those on low-carbohydrate or other specific diets; results derived from linear-regression analysis with FBG levels as the main outcome and mushroom consumption as the independent factor are presented as $\beta$-coefficients [ $\beta$-coef (95\% CI)]; Model 1 was unadjusted; Model 2 was adjusted for age and energy intake; Model 3 was adjusted for confounders of Model 2 plus physical activity, smoking habits, social status, systolic and diastolic blood pressure, lipid profile markers and body mass index; Model 4 was adjusted for confounders of Model 3 plus dietary selenium; Model 5 was adjusted for confounders of Model 4 plus intake of grains, pasta, nuts, seeds, legumes, potatoes, poultry and fish, and total antioxidant (food antioxidant content) score.

a Identical to Model 3.

had higher social status and were less frequently hypertensive, and also had higher energy intakes, higher intakes of other food sources of selenium and higher FAC scores compared with men in the lowest tertile $(P<0.05$ for all). In addition, men in the highest category of mushroom consumption also had greater intakes of selenium and more frequently exceeded the RDA of $55 \mu \mathrm{g} /$ day $(P<0.05$ for both). Indeed, in the total male population, $67.8 \%$ exceeded the RDA. On the other hand, univariate analysis of all men indicated no differences in glucose levels across tertiles of mushroom consumption ( $P>0.05$ for both).

Women in the highest tertile of mushroom consumption were younger, more physically active, more frequently current smokers, had higher social status and were less hypertensive, while also having lower energy intakes, higher intakes of other food sources of selenium and higher FAC scores compared with women in the lowest tertile $(P<0.05$ for all $)$. Moreover, women in the highest category of mushroom consumption had greater intakes of selenium and more frequently exceeded the RDA of $55 \mu \mathrm{g} /$ day $(P<0.05$ for both). In particular, $55.6 \%$ exceeded the RDA in the total female study population. However, no significant difference in glucose levels was observed on univariate analysis across tertiles of mushroom consumption.

Univariate analysis indicated that, except for potatoes, nuts and legumes $(P>0.05)$, the food sources of selenium were positively and significantly correlated with FBG levels in men (Pearson's $r>0, P<0.05$ ). In women the correlations were not evident for most food groups, and only legumes and chicken demonstrated a positive trend in relation to glucose (Pearson's $r>0, P<0.05$ ).

To establish whether the link between mushroom and dietary selenium intakes with FBG was linear, further analyses were conducted. However, on adding squared and cubed terms of both dietary data at all levels of adjustment, no significant associations were observed $(P>0.05$ for all).
Table 3 shows the association between mushroom and dietary selenium intakes with FBG levels. In men, an increase of one portion $(30 \mathrm{~g})$ per week in mushroom consumption was associated with a $0.43-0.55 \mathrm{mmol} / \mathrm{L}$ increase in $\mathrm{FBG}$ at different levels of adjustment (Models $2-5 ; \beta$-coef $>0, P<0.05$ for all). This positive association can also be seen on Fig. 1a.

The same analyses of women indicated similar positive associations, albeit not significant ones (Table 3; Fig. 1b). In particular, there was a significant gender interaction in the relationship between mushroom consumption and glucose levels $(P<0.05)$.

Median selenium intakes in men and women were 62.6 (51.7, $75.7)$ and $57.4(47.0,69.8) \mathrm{g} / \mathrm{week}$, respectively $(P<0.001$ by $t$ test). In both men and women, dietary selenium was positively associated with blood glucose in both unadjusted and fully adjusted models ( $P<0.05$ for all; Table 3; Fig. 2$)$. In particular, an increase of $50 \mu \mathrm{g} /$ day (close to the RDA) in dietary selenium in men and women was associated with increases of 0.14 and $0.11 \mathrm{mmol} / \mathrm{L}$, respectively, in FBG levels in the multivariate model (Model 5; $P<0.05$ for all).

The same analysis for selenium intakes and FBG levels, but using the residuals method for energy intake, produced similar findings. In particular, the residuals predicted by regression analysis with dietary selenium as the dependent factor and energy intake as the independent factor were significantly and positively associated with FBG at all levels of adjustment $(\beta$-coef $>0$, $P<0.05$ for all).

For the entire Moli-sani population $(m e n=9667$, women $=10,625$ ), including diabetics and those on lowcarbohydrate or other specific diets, logistic-regression analyses with the presence of diabetes as the outcome and mushroom or selenium intake as the independent factor were also conducted (Table 4). Results indicated that, in the fully adjusted model (Model 5), men and women consuming $>28 \mathrm{~g} /$ week (higher

Please cite this article in press as: Pounis G, et al. Mushroom and dietary selenium intakes in relation to fasting glucose levels in a free-living Italian adult population: The Moli-sani Project. Diabetes Metab (2013), http://dx.doi.org/10.1016/j.diabet.2013.09.007 


+Model
DIABET 5651-9

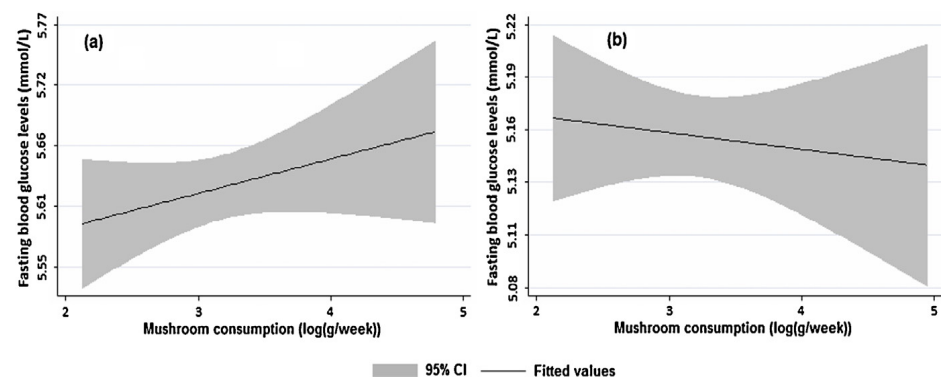

Fig. 1. Graphs representing the relationship between increases in mushroom consumption (one 30-g portion per week) and increases in fasting blood glucose levels in men (a) and women (b)
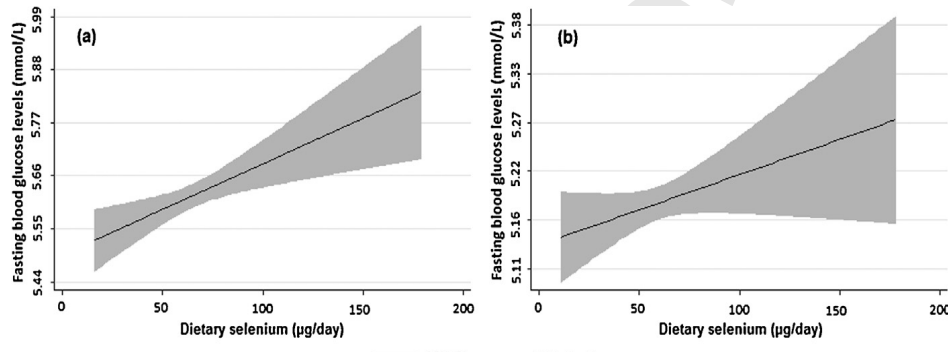

Fig. 2. Graphs representing the relationship between increases in median selenium intakes in men (a) and women (b) in relation to fasting blood glucose levels

Table 4

Mushroom consumption and dietary selenium in relation to diabetes prevalence in the Moli-sani population.

\begin{tabular}{|c|c|c|c|c|c|}
\hline & \multicolumn{5}{|c|}{ Likelihood of diabetes, OR $(95 \% \mathrm{CI})$} \\
\hline & $\begin{array}{l}\text { Model } 1 \\
\text { (crude) }\end{array}$ & $\begin{array}{l}\text { Model } 2 \\
\text { (multiple-adjusted) }\end{array}$ & $\begin{array}{l}\text { Model } 3 \\
\text { (multiple-adjusted) }\end{array}$ & $\begin{array}{l}\text { Model } 4 \\
\text { (multiple-adjusted) }\end{array}$ & $\begin{array}{l}\text { Model } 5 \\
\text { (multiple-adjusted) }\end{array}$ \\
\hline \multicolumn{6}{|l|}{$\operatorname{Men}(n=9667)$} \\
\hline \multicolumn{6}{|c|}{ Categories of mushroom consumption } \\
\hline $14-28$ (g/week) & $1.01(0.85,1.20)$ & $1.13(0.94,1.36)$ & $1.20(0.99,1.46)$ & $1.17(0.97,1.43)$ & $1.18(0.97,1.43)$ \\
\hline$>28$ (g/week) & $1.05(0.88,1.26)$ & $1.28(1.07,1.540)$ & $1.33(1.10,1.62)$ & $1.27(1.05,1.55)$ & $1.27(1.05,1.55)$ \\
\hline \multicolumn{6}{|l|}{ Women $(n=10,625)$} \\
\hline \multicolumn{6}{|c|}{ Categories of mushroom consumption } \\
\hline$<14$ (g/week) & & & Reference category & & \\
\hline 14-28 (g/week) & $1.06(0.83,1.34)$ & $1.29(1.00,1.64)$ & $1.35(1.03,1.76)$ & $1.30(1.00,1.70)$ & $1.30(1.00,1.70)$ \\
\hline$>28$ (g/week) & $1.04(0.82,1.33)$ & $1.43(1.11,1.83)$ & $1.48(1.13,1.94)$ & $1.39(1.06,1.82)$ & $1.38(1.05,1.81)$ \\
\hline \multicolumn{6}{|l|}{ Men $(n=9667)$} \\
\hline \multicolumn{6}{|c|}{ Categories of dietary selenium } \\
\hline$<50$ ( $\mu \mathrm{g} /$ day $)$ & & . & Reference category & & \\
\hline $50-60(\mu \mathrm{g} /$ day $)$ & $0.83(0.70,0.98)$ & $1.16(0.97,1.39)$ & $1.19(0.96,1.49)$ & $-^{\mathrm{a}}$ & $1.19(0.95,1.48)$ \\
\hline $61-75$ ( $\mu \mathrm{g} /$ day $)$ & $0.66(0.55,0.79)$ & $1.12(0.92,1.36)$ & $1.15(0.90,1.47)$ & $--^{\mathrm{a}}$ & $1.14(0.89,1.46)$ \\
\hline$>75$ ( $\mu \mathrm{g} /$ day $)$ & $0.70(0.59,0.84)$ & $1.51(1.21,1.88)$ & $1.35(1.03,1.77)$ & $--^{\mathrm{a}}$ & $1.33(1.01,1.77)$ \\
\hline \multicolumn{6}{|l|}{ Women $(n=10,625)$} \\
\hline \multicolumn{6}{|c|}{ Categories of dietary selenium } \\
\hline$<50$ ( $\mu \mathrm{g} /$ day $)$ & (e & & Reference category & & \\
\hline $50-60(\mu \mathrm{g} /$ day $)$ & $0.84(0.67,1.05)$ & $1.29(1.02,1.63)$ & $1.26(0.92,1.73)$ & $-{ }^{\mathrm{a}}$ & $1.22(0.89,1.67)$ \\
\hline $61-75$ ( $\mu \mathrm{g} /$ day $)$ & $0.71(0.56,0.89)$ & $1.36(1.05,1.77)$ & $1.33(0.95,1.87)$ & $--^{\mathrm{a}}$ & $1.26(0.89,1.78)$ \\
\hline$>75$ ( $\mu \mathrm{g} /$ day $)$ & $0.74(0.59,0.93)$ & $1.86(1.40,2.46)$ & $1.85(1.28,2.68)$ & $-{ }^{\mathrm{a}}$ & $1.71(1.17,2.51)$ \\
\hline
\end{tabular}

CI: confidence intervals. Results derived by logistic-regression analysis with diabetes as main outcome and mushroom consumption as independent factor are presented as odds ratios (OR); Model 1 was unadjusted; Model 2 was adjusted for age and energy intake; Model 3 was adjusted for confounders of Model 2 plus physical activity, smoking habits, social status, systolic and diastolic blood pressure, lipid profile markers and body mass index; Model 4 was adjusted for confounders of Model 3 plus dietary selenium; Model 5 was adjusted for confounders of Model 4 plus intake of grains, pasta, nuts, seeds, legumes, potatoes, poultry and fish, and total antioxidant (food antioxidant content) score.

${ }^{\text {a }}$ Identical to Model 3.

Please cite this article in press as: Pounis G, et al. Mushroom and dietary selenium intakes in relation to fasting glucose levels in a free-living Italian adult population: The Moli-sani Project. Diabetes Metab (2013), http://dx.doi.org/10.1016/j.diabet.2013.09.007 
tertile) had a $27 \%$ (OR: $1.27,95 \%$ CI: $1.05,1.55)$ and $38 \%$ (OR: 1.38, 95\% CI: 1.05, 1.81) greater likelihood, respectively, of the presence of diabetes compared with those in the lowest tertiles.

Results of the logistic-regression analysis for dietary selenium also showed positive associations between high dietary selenium and diabetes prevalence in both genders (Table 4). In particular, after adjustments (Models 2-5), associations were significant only in the highest quartile of selenium intake ( $>75 \mu \mathrm{g} /$ day; OR: $>1, P<0.05)$. In contrast, on crude analysis (Model 1) the association was negative and significant in all categories of intake (OR: $<1, P<0.05)$.

\section{Discussion}

In the present study, mushroom consumption was positively associated with FBG levels in non-diabetic men not on special diets, whereas no such association was found in women.

Median consumption of mushrooms in both men and women was $14.0 \mathrm{~g} /$ week, which was lower than that reported by Hong et al. [32] in a sample of Korean women with breast cancer, where values ranged from 49 to $80 \mathrm{~g} /$ week.

However, it is well known that for the past several thousands of years, people in the Far East have been major consumers of mushrooms as a source of both nutrition and medicine [8]. Indeed, in case-control studies an inverse relationship between mushroom consumption and cancer has been observed [32-35]. Furthermore, three kinds of carcinostatic polysaccharide agents have been developed in Japan:

- 'lentinan' from the fruiting bodies of shiitake;

- 'krestin' from the cultured mycelia of kawaratake;

- 'schizophyllan' (Sonifilan) from the cultured broth products of suehirotake [2].

Studies in animal models and human cells have determined the antioxidant mechanisms by which the consumption of mushroom extracts may be of benefit against diseases related to oxidative stress [3-7]. A small clinical trial published by Chang et al. [9] suggested both hypoglycaemic and hypodyslipidaemic effects with mushroom supplementation in a diabetic sample population. However, evidence for the protective effects of mushroom consumption on human health is still limited.

Nevertheless, our present results have shown that, in an adult Italian population free of diabetes, increased mushroom intake was associated with higher glucose levels. In particular, an increase of one $30 \mathrm{~g}$ portion of mushrooms per week was associated with a $0.47 \mathrm{mmol} / \mathrm{L}$ increase in FBG in men (fully adjusted model). The association of mushroom consumption and FBG appears to be linear as neither squared nor cubed models indicated significant results. In addition, the association was limited to only men, suggesting a possible gender difference. Recent studies have discussed the different levels of oxidative stress found in men and women; whole-body reactive oxygen species (ROS) production appear to be higher in healthy young men than in women [30]. Moreover, differences in the sensitivity of men vs. women to antioxidant changes have previously been observed in the Moli-sani population [14]. However, the lack of a significant association between mushrooms and glucose in women might be due to the more limited range of mushroom consumption in this particular group.

Mushrooms can be characterized as among the richest plant sources of selenium $[13,14]$, with contents recently calculated as $11.9 \mu \mathrm{g}$ per $100 \mathrm{~g}$ by the present authors' group [14]. However, in contrast to the limited evidence of health-protective effects with mushroom extracts mostly coming from the Far East, there have recently been evidence-based conclusions suggesting detrimental effects of serum selenium on diabetes [15-17]. Cross-sectional results from the third US National Health and Nutrition Examination Survey (NHANES III) found alarming results for a positive association of serum selenium levels with the prevalence of diabetes [15,16], and similar results have been obtained in our present study population wherein dietary selenium was positively related to FBG in both genders, and $67.8 \%$ and $55.6 \%$ of men and women, respectively, exceeded the RDA of $55 \mu \mathrm{g} / \mathrm{day}$ for selenium intake.

However, it is of interest to note the similarities between the course of studies of mushroom and selenium effects on health Indeed, before the recent findings supporting an association between selenium status and diabetes prevalence and incidence, several reports of its association with cancer prevention had been published [36,37].

For this reason, to determine whether the association between mushroom intake and glucose levels might be explained by its selenium content or confounded by other foods containing selenium, our analyses were also adjusted for the relevant variables. However, no changes were observed in the demonstrated associations. Thus, these results support the independence of the associations of mushroom and selenium intakes with FBG levels.

It could be hypothesized that something other than selenium content might account for the positive association of mushrooms with glucose levels. Recently, research evidence has shown that mushrooms can accumulate high concentrations of toxic metallic elements, metalloids, radionuclides and other heavy metals [38], and epidemiological studies suggest that some of them, such as mercury and cadmium, might increase the risk of insulin resistance [39] or diabetes [40,41]. In vitro and in vivo studies have also confirmed a link between toxic heavy metals and diabetes. In particular, increased concentrations of such metals can cause beta-cell death and decrease insulin secretion from beta-cells [42-44].

Nevertheless, mushrooms, aside from their heavy metal contents, are low in calories and a good source of B vitamins, so their importance in a balanced way of eating is not negligible. Future research, including studies of biological mechanisms, cohorts and clinical trials, are now needed to clarify the positive association between mushroom consumption and glucose levels reported here.

To further investigate the similar positive association with selenium, the relationship between mushroom and selenium intake with diabetes prevalence in our total study population was also analyzed. After adjusting for possible confounders, 
those men and women consuming higher quantities of mushrooms (>28 g/week) had a $27 \%$ and $38 \%$, respectively, greater likelihood of diabetes compared with those in the lowest tertiles of consumption. Similarly, high selenium intake was associated with a greater likelihood of having diabetes in both men and women.

Despite the original findings, the present study nevertheless has some limitations. First, the cross-sectional design prohibits determination of causality. Second, the mechanisms by which clinical biomarkers affect or are affected by mushroom intake can only be speculated and, as the study was not designed to investigate biological mechanisms, further studies are needed to that end. Third, possible errors due to misreporting by subjects have to be acknowledged, as well as the lack of data derived by NAF software (nutrient analysis of the FFQ) for individual food contributions to total selenium intakes, which limit our results. Finally, there were difficulties in exposure assessment through the use of FFQs, although there is evidence that this has not strongly affected the validity of other similar studies [45].

In conclusion, the present study found a positive significant relationship between mushroom and selenium intakes and FBG levels, although the independence of these associations has led to a hypothesis of the possible role of the toxic heavy metal contents of mushrooms on glucose levels. Further prospective studies are needed to confirm these findings. Their replication is of the utmost importance for public health promotion, as mushrooms have long been considered a healthy food group, especially for people with diabetes.

\section{Disclosure of interest}

The authors declare that they have no conflicts of interest concerning this article.

\section{Uncited references}

$[19,20]$.

\section{Acknowledgments}

The authors thank the Associazione Cuore Sano (Healthy Heart Association) ONLUS (Campobasso, Italy), Derby Blue (San Lazzaro di Savena, Bologna, Italy), Caffè Monforte (Campobasso, Italy) and Sepinia SpA (Sepino, Italy) for their support of the Moli-sani Project. None of the funding organizations or sponsors played any role in the design or conduct of the study, nor in the collection, management, analysis or interpretation of the data, or in the preparation, review or approval of the manuscript.

The Moli-sani research group would like to thank Dr Vottorio Krogh from Istituto Nazionale dei Tumori (National Cancer Institute) of Milan, Italy, for his contribution to the dietary anal-

The authors' contributions were as follows: G.P. provided the idea, performed the analyses and wrote the manuscript; S.C. managed the database; M.P., A.d.C. and F.Z. collected the data; A.F.d.C. reviewed the statistical analysis; M.B.D, G.d.G and L.I. reviewed drafts of the manuscripts; S.S. and M.V. contributed to the nutritional analyses and critically reviewed the manuscript; L.I. was the guarantor of this work and, as such, had full access to all the data in the study, and took full responsibility for the integrity of the data and accuracy of the data analysis.

Sources of support: The Moli-sani cohort recruitment was supported by an unrestricted grant from Pfizer Foundation (Rome, Italy), the Italian Ministry of University and Research (MIUR, Rome, Italy), three-year Program of Research Decree no. 1588, and Instrumentation Laboratory (Milan, Italy).

\section{References}

[1] Chang ST. Mushrooms and mushroom cultivation. eLS; 2001.

Q3 48 mushrooms. Food Rev Int 1995;11:69-81.

[3] Ajith TA, Janardhanan KK. Indian medicinal mushrooms as a source of antioxidant and antitumor agents. J Clin Biochem Nutr 2007:40:157-62.

[4] Bobek P, Galbavy S. Effect of pleuran (beta-glucan from Pleurotus ostreatus) on the antioxidant status of the organism and on dimethylhydrazine-induced precancerous lesions in rat colon. Br J Biomed Sci 2001;58:164-8.

[5] Cheung LM, Cheung PCK. Mushroom extracts with antioxidant activity against lipid peroxidation. Food Chem 2005;89:403-9.

[6] Song YS, Kim SH, Sa JH, Jin C, Lim CJ, Park EH. Antiangiogenic, antioxidant and xanthine oxidase inhibition activities of the mushroom Phellinus linteus. J Ethnopharmacol 2003;88:113-6.

[7] Matsuzawa T. Studies on antioxidant effects of culinary-medicinal bunashimeji mushroom Hypsizygus marmoreus (Peck) Bigel. (Agaricomycetideae). Int J Med Mushrooms 2006:8:245-50.

[8] Ikekawa T. Beneficial effects of edible and medicinal mushrooms on health care. Int J Med Mushrooms 2001;3:291-8.

[9] Chang JH, Kim MS, Kim JY, Choi WH, Lee SS. Effects of mushroom supplementation on blood glucose concentration, lipid profile, and antioxidant enzyme activities in patients with type 2 diabetes mellitus. Korean J Nutr 2007;40:327-33.

[10] Sullivan R, Smith JE, Rowan NJ. Medicinal mushrooms and cancer therapy: translating a traditional practice into Western medicine. Perspect Biol Med 2006;49:159-70.

[11] Guillamón E, García-Lafuente A, Lozano M, D’Arrigo M, Rostagno MA, Villares A, et al. Edible mushrooms: role in the prevention of cardiovascular diseases. Fitoterapia 2010;81:715-23.

[12] Jeong SC, Jeong YT, Yang BK, Islam R, Koyyalamudi SR, Pang G, et al. White button mushroom (Agaricus bisporus) lowers blood glucose and cholesterol levels in diabetic and hypercholesterolemic rats. Nutr Res 2010;30:49-56

[13] United States Department of Agriculture (USDA). USDA reports by single nutrients [article online]. Available from https://www.ars. Q usda.gov/SP2UserFiles/Place/12354500/Data/SR25/nutrlist/sr25w317.pdf. Accessed February 2013.

[14] Pounis G, Costanzo S, di Giuseppe R, de Lucia F, Santimone I, Sciarretta A, et al. Consumption of healthy foods at different content of antioxidant vitamins and phytochemicals and metabolic risk factors for cardiovascular disease in men and women of the Moli-sani study. Eur J Clin Nutr 2013;67:207-13.

[15] Laclaustra M, Navas-Acien A, Stranges S, Ordovas JM, Guallar E. Serum selenium concentrations and diabetes in US adults: National Health and Nutrition Examination Survey (NHANES) 2003-2004. Environ Health Perspect 2009;117:1409-13.

[16] Bleys J, Navas-Acien A, Guallar E. Serum selenium and diabetes in US adults. Diabetes Care 2007;30:829-34.

[17] Stranges S, Marshall JR, Natarajan R, Donahue RP, Trevisan M, Combs $\mathrm{GF}$, et al. Effects of long-term selenium supplementation on the incidence of type 2 diabetes: a randomized trial. Ann Intern Med 2007;147:217-23.

[18] Bleys J, Navas-Acien A, Guallar E. Selenium and diabetes: more bad news for supplements. Ann Intern Med 2007;147:271-2. 
38 [19] Mueller AS, Mueller K, Wolf NM, Pallauf J. Selenium and diabetes: an enigma? Free Radic Res 2009;43:1029-59.

[20] Rocourt CRB, Cheng WH. Selenium supranutrition: are the potential benefits of chemoprevention outweighed by the promotion of diabetes and insulin resistance? Nutrients 2013;5:1349-65.

[21] Steinbrenner H, Speckmann B, Pinto A, Sies H. High selenium intake and increased diabetes risk: experimental evidence for interplay between selenium and carbohydrate metabolism. J Clin Biochem Nutr 2011;48:40-5, http://dx.doi.org/10.3164/jcbn.11-002FR10.

[22] Iacoviello L, Bonanni A, Costanzo S, De Curtis A, Di Castelnuovo A, Olivieri M, et al. The Moli-Sani Project, a randomized, prospective cohort study in the Molise region in Italy, design, rationale and objectives. Ital J Public Health 2007;4:110-8.

[23] Centritto F, Iacoviello L, di Giuseppe R, De Curtis A, Costanzo S, Zito $\mathrm{F}$, et al. Dietary patterns, cardiovascular risk factors and C-reactive protein in a healthy Italian population. Nutr Metab Cardiovasc Dis 2009;19: 697-706.

[24] Ada I. Classification and Diagnosis. Diabetes Care 2013;36: S13.

[25] Nuorti JP, Butler JC, Farley MM, Harrison LH, McGeer A, Kolczak MS, et al. Cigarette smoking and invasive pneumococcal disease. Active Bacterial Core Surveillance Team. N Engl J Med 2000;342:681-9.

[26] Ainsworth BE, Haskell WL, Whitt MC, Irwin ML, Swartz AM, Strath SJ, et al. Compendium of physical activities: an update of activity codes and MET intensities. Med Sci Sports Exerc 2000;32:498-504.

[27] Pisani P, Faggiano F, Krogh V, Palli D, Vineis P, Berrino F. Relative validity and reproducibility of a food frequency dietary questionnaire for use in the Italian EPIC centers. Int J Epidemiol 1997;26:152-60.

[28] Pala V, Sieri S, Palli D, Salvini S, Berrino F, Bellegotti M, et al. Diet in the Italian EPIC cohorts: presentation of data and methodological issues. Tumori 2003;89:594-607.

[29] Istituto Nazionale di Ricerca per gli Alimenti e la Nutrizione. Italian food composition tables [article online]. Available from http://www. inran.it/646/tabelle_di_composizione_degli_alimenti.html. Accessed 20 December 2011.

[30] Food and Nutrition Board, Institute of Medicine. Dietary reference intakes for vitamin C, vitamin E, selenium, and carotenoids. A report of the Panel on Dietary Antioxidants and Related Compounds, Subcommittees on Upper Reference Levels of Nutrients and Interpretation and Uses of Dietary Reference Intakes and the Standing Committee on the Scientific Evaluation of Dietary Reference Intakes. Washington, DC: National Academy Press; 2000 .
[31] Willett WC, Howe GR, Kushi LH. Adjustment for total energy intake in epidemiologic studies. Am J Clin Nutr 1997;65:1220S-8S [Discussion 1229S-1231S].

[32] Hong SA, Kim K, Nam SJ, Kong G, Kim MK. A case-control study on the dietary intake of mushrooms and breast cancer risk among Korean women Int J Cancer 2008;122:919-23.

[33] Lee SA, Kang D, Nishio H, Lee MJ, Kim DH, Han W, et al. Methylenetetrahydrofolate reductase polymorphism, diet, and breast cancer in Korean women. Exp Mol Med 2004;36:116-21.

[34] Zhang M, Huang J, Xie X, Holman CD. Dietary intakes of mushrooms and green tea combine to reduce the risk of breast cancer in Chinese women. Int J Cancer 2009;124:1404-8.

[35] Ide T, Tsutsui H, Ohashi N, Hayashidani S, Suematsu N, Tsuchihashi $\mathrm{M}$, et al. Greater oxidative stress in healthy young men compared with premenopausal women. Arterioscler Thromb Vasc Biol 2002;22:438-42.

[36] Willett WC, Stampfer MJ. Selenium and cancer. BMJ 1988;297:573-4.

[37] Rayman MP. Selenium in cancer prevention: a review of the evidence and mechanism of action. Proc Nutr Soc 2005;64:527-42.

[38] Campos JA, Tejera NA, Sánchez CJ. Substrate role in the accumulation of heavy metals in sporocarps of wild fungi. Biometals 2009;22:835-41.

[39] Chang JW, Chen HL, Su HJ, Liao PC, Guo HR, Lee CC. Simultaneous exposure of non-diabetics to high levels of dioxins and mercury increases their risk of insulin resistance. J Hazard Mater 2011;185:749-55.

[40] He K, Xun P, Liu K, Morris S, Reis J, Guallar E. Mercury exposure in young adulthood and incidence of diabetes later in life: the CARDIA Trace Element Study. Diabetes Care 2013;36:1584-9.

[41] Edwards JR, Prozialeck WC. Cadmium, diabetes and chronic kidney disease. Toxicol Appl Pharmacol 2009;238:289-93.

[42] Chen YW, Huang CF, Yang CY, Yen CC, Tsai KS, Liu SH. Inorganic mercury causes pancreatic beta-cell death via the oxidative stress-induced apoptotic and necrotic pathways. Toxicol Appl Pharmaco 2010;243:323-31.

[43] Chen YW, Huang CF, Tsai KS, Yang RS, Yen CC, Yang CY, et al. Methylmercury induces pancreatic beta-cell apoptosis and dysfunction. Chem Res Toxicol 2006;19:1080-5.

[44] Chen YW, Huang CF, Tsai KS, Yang RS, Yen CC, Yang CY, et al. The role of phosphoinositide 3-kinase/Akt signaling in low-dose mercury-induced mouse pancreatic beta-cell dysfunction in vitro and in vivo. Diabetes 2006;55:1614-24.

[45] Dennert G, Zwahlen M, Brinkman M, Vinceti M, Zeegers MP, Horneber M. Selenium for preventing cancer. Cochrane Database Syst Rev 2011;5:CD005195. 
Chapter 5.

Folate intake and folate serum levels in men and women from two European populations: The IMMIDIET project

Pounis G, Di Castelnuovo AF, de Lorgeril M, Krogh V, Siani A, Arnout J, Cappuccio FP, van

Dongen M, Zappacosta B, Donati MB, de Gaetano G, lacoviello L, on behalf of the European Collaborative Group of the IMMIDIET Project. Folate intake and folate serum levels in men and women from two European populations: The IMMIDIET project. Nutrition, In Press. 


\section{Accepted Manuscript}

Folate intake and folate serum levels in men and women from two European populations: The IMMIDIET project

George Pounis, MSc Augusto F. Di Castelnuovo, PhD Michel de Lorgeril, MD Vittorio Krogh, MD Alfonso Siani, MD Jozef Arnout, MD, PhD Francesco P. Cappuccio, MD Martien van Dongen, PhD Bruno Zappacosta, MD Maria Benedetta Donati, MD, PhD Giovanni de Gaetano, MD, PhD Licia lacoviello, MD, PhD

PII: S0899-9007(13)00542-X

DOI: 10.1016/j.nut.2013.11.014

Reference: $\quad$ NUT 9167

To appear in: Nutrition

Received Date: 22 April 2013

Revised Date: 18 November 2013

Accepted Date: 21 November 2013

Please cite this article as: Pounis G, Di Castelnuovo AF, de Lorgeril M, Krogh V, Siani A, Arnout J, Cappuccio FP, van Dongen M, Zappacosta B, Donati MB, de Gaetano G, lacoviello L, , Folate intake and folate serum levels in men and women from two European populations: The IMMIDIET project, Nutrition (2014), doi: 10.1016/j.nut.2013.11.014.

This is a PDF file of an unedited manuscript that has been accepted for publication. As a service to our customers we are providing this early version of the manuscript. The manuscript will undergo copyediting, typesetting, and review of the resulting proof before it is published in its final form. Please note that during the production process errors may be discovered which could affect the content, and all legal disclaimers that apply to the journal pertain. 
1 Folate intake and folate serum levels in men and women

2 from two European populations: The IMMIDIET project

3 Running title: Folate intake and levels in 2 EU countries

4

5 George Pounis $\mathrm{MSc}^{1}$, Augusto F Di Castelnuovo $\mathrm{PhD}^{1}$, Michel de Lorgeril, $\mathrm{MD}^{2}$,

6 Vittorio Krogh, $\mathrm{MD}^{3}$, Alfonso Siani, $\mathrm{MD}^{4}$, Jozef Arnout, $\mathrm{MD}, \mathrm{PhD}^{5}$, Francesco $\mathrm{P}$

7 Cappuccio, $\mathrm{MD}^{6}$, Martien van Dongen, $\mathrm{PhD}^{7}$, Bruno Zappacosta, $\mathrm{MD}^{8}$, Maria

8 Benedetta Donati, $\mathrm{MD}, \mathrm{PhD}^{1}$, Giovanni de Gaetano, $\mathrm{MD}, \mathrm{PhD}^{1}$, Licia Iacoviello, $\mathrm{MD}$,

$9 \mathrm{PhD}^{1}$, on behalf of the European Collaborative Group of the IMMIDIET Project

$11{ }^{1}$ Department of Epidemiology and Prevention, IRCCS Istituto Neurologico

12 Mediterraneo Neuromed, Pozzilli (IS), Italy

$132{ }^{2}$ Université Joseph Fourier-CNRS, Faculté de Médecine, La Tronche, France

$14{ }^{3}$ Istituto Nazionale dei Tumori, Milan, Italy

$15{ }^{4}$ Institute of Food Sciences, CNR, Avellino, Italy

$16 \quad{ }^{5}$ Katholieke Universiteit Leuven, Belgium

$17{ }^{6}$ Warwick Medical School, Coventry, United Kingdom

$18{ }^{7}$ Maastricht University, The Netherlands

$19{ }^{8}$ U.O.C. Laboratorio Analisi, Fondazione di Ricerca e Cura "Giovanni Paolo II",

20 Università Cattolica del Sacro Cuore, Campobasso, Italy

21 *IMMIDIET Project Investigators are listed in the Appendix 
23 Corresponding Author: Licia Iacoviello, MD, PhD, Department of Epidemiology

24 and Prevention, IRCCS Istituto Neurologico Mediterraneo Neuromed, Via

25 dell’Elettronica. 86077 Pozzilli, (IS), Italy. E-mail: licia.iacoviello@ neuromed.it. Tel:

$26+39.0865929664$.

27

28 Author contribution

29 Conception and design of the study: MdL, VK, AS, JA, FPC, LI. Generation,

30 collection, assembly, analysis and/or interpretation of data: GP, AFDC, MdL, VK,

31 AS, JA, FPC, MvD, LI. Drafting or revision of the manuscript: GP, AFDC, MdL, VK,

32 AS, JA, FPC, MvD, MBD, GdG, LI. Approval of the final version of the manuscript:

33

GP, AFDC, MdL, VK, AS, JA, FPC, MvD, MBD, GdG, LI. 
34 ABSTRACT

35 Objective: Folate status has been associated with neural tube defects and cerebrovascular disease. We aimed at evaluating possible differences in folate status in two EU Countries and to assess their possible association with dietary patterns and/or other lifestyles. Methods and Procedures: In the framework of the EUfunded IMMIDIET Project, 1,068 subjects (534 man-woman pairs), aged 26-64 years, were enrolled in Italy and UK. 1-year-recall Food Frequency Questionnaire was used to evaluate dietary intake. Reduced Rank Regression-analysis was used to derive a dietary pattern better describing high dietary folate intake. Results: $11.3 \%$ of the Italians and $45.1 \%$ of the British exceeded the optimal dietary folate intake of $400 \mu \mathrm{g} /$ day (Recommended Dietary Allowance). $66.7 \%$ and $22.1 \%$ of Italian and UK women, respectively, all at childbearing age, had folate serum levels lower than $6.62 \mathrm{ng} / \mathrm{ml}(\mathrm{P}=0.01)$. The percentage of total variance of dietary folate intake explained by food group consumption was $14.2 \%$ and $16.3 \%$ in Italy and UK, respectively. Reduced Rank Regression-analysis indicated a healthy pattern which was positively associated with folate serum levels in both Countries (for all $\beta$-coef $>0$, $\mathrm{P}<0.001): 100 \mu \mathrm{g} /$ day increase in dietary folate intake was associated with $13.8 \%$ and $10.5 \%$ increase in folate serum levels in the Italian and English population, respectively (for $100 \mu \mathrm{g} / \mathrm{day}$ increase $\mathrm{e}^{\beta-\mathrm{coef}}=1.138$ and $1.105, \mathrm{P}<0.001$ ). Smoking habit was negatively but physical activity positively associated with folate serum levels $(\mathrm{P}<0.05)$. Conclusion(s): An inadequate dietary folate intake and subsequent serum levels were observed in Italian subjects. High consumption of food sources of folate was positively associated with folate serum levels, explaining a good proportion of its variability. 
58 Key words: folate; folate status; diet; dietary patterns, cardiovascular disease,

59 neurovascular disease 


\section{INTRODUCTION}

Folate is a water-soluble B-vitamin and one of the micronutrients included in the Mediterranean diet [1]. Reduced folate levels have been associated with cardiovascular disease [2-4], possibly through increase in homocysteine; however, trials aiming at reducing homocysteine with folate supplementation have shown overall negative results [5], excepted for a reduction in stroke prevention [6, 7].

More consistent are the data on the association between low folate levels in pregnant women and neural tube defects (NTD) or other adverse birth outcomes [8]. The increase in serum folate with the diet or by supplementation drastically reduces the birth prevalence of NTDs. In the US, Canada, Chile and Costa Rica, which all made mandatory the fortification of flour between 1998 and 2000, the drop in NTD rates among live new-born babies was between 23 and 78\% [9]. However, the fortification is still non-mandatory in several European Countries mainly due to a concern that folate fortification may harm people with undiagnosed vitamin B12 deficiency [10].

In Particular in Italy food folate fortification is not mandatory and folate supplementation of women at childbearing age or health promotion strategies targeted at increasing intake of dietary sources, are not a major public health issues.

On the contrary, in England public health promotion strategies force consumers to prefer fortified foods as a health protection choice. In 2007 UK Food Standards Agency considering the report of Scientific Advisory Committee on Nutrition (SACN) [11] recommended the mandatory fortification of bread or flour with folate in order to reduce the risk of neural tube defects in foetuses. However, no such decision has already been taken by the Health Ministers. 
There is a wide list of foods containing folate, however the relation between folate food intake and folate serum levels is not so strong and there is limited evidence on which food source could really help the population introducing the appropriate folate daily amount to achieve the desired serum levels [12]. Moreover, it is of interest to better understand factors, beside diet, that might affect folate levels.

This study aims firstly at describing the present status of dietary folate intake and serum levels in a population of men and women from Italy and UK, a Southern and a Northern European Country, respectively, with no mandatory food fortification strategy for folate. Secondly, it aims at identifying food patterns that better describe a high folate intake in these two Countries and at evaluating if and how such patterns are associated with folate serum levels.

96 MATERIALS AND METHODS

97 Study population

98 The IMMIDIET Project $[13,14]$ and subject recruitment procedures have been 99 previously described. The IMMIDIET study is a population-based cross-sectional study; apparently healthy pairs were man-woman spouses or partners living together, recruited through local general practices. To protect against selection bias, the selection of eligible pairs was randomized in each centre. Between October 2001 and October 2003, 271 pairs in the Abruzzo region in Italy and 263 in S-W London in United Kingdom (UK) aged 26-64 years (mean \pm SD: men $48 \pm 7$, women $45 \pm 7$ ) were randomly enrolled $[13,14]$. The participation rates were $85 \%$ in Italy and $90 \%$ in UK. The ethical committees of all participating institutions using the ethical standards laid down in the 1964 Declaration of Helsinki and its later amendments approved the 
study. All study participants agreed to participate by written informed consent prior to their inclusion into the study.

\section{Measurements}

Interviews were taken using a standardised questionnaire previously adopted [15].

Subjects were classified as non-smokers (if they had never smoked cigarettes), ex-smokers (if they had smoked cigarettes in the past), and current smokers if they were currently smoking on a regular daily basis one or more cigarettes per day. Physical activity rate was assessed by a standardised questionnaire [15]. Subjects were grouped in 2 categories of physical activity ("low" or "high") according to the median rate of each population. Socio-economic status was defined as a score (0-5) based on 3 variables: education, job and housing. The higher the score, the higher was the level of socio-economic status. Participants were grouped in 2 categories of socioeconomic status ("low" or "high") according to the median of each population. Women were divided into groups of pre-menopause and menopause according to their reporting.

Body weight and height were measured on a standard beam balance scale with an attached ruler, in subjects wearing no shoes and only light indoor clothing. Body mass index (BMI) was calculated as weight in kilograms divided by the square of the height in meters $(\mathrm{kg} / \mathrm{m} 2)$. Blood samples were obtained between 7.00 and 10.00 a.m. from subjects who had been fasting overnight and had refrained from smoking for at least 6 hours. 
Folate serum levels were determined by a chemoluminescent assay at microparticles capture (CMIA), Axsym (ABBOTT) (adequate levels, folate $>6.62$ $\mathrm{ng} / \mathrm{ml}$ ) [16]. The assay sensitivity was lower than $0.8 \mathrm{ng} / \mathrm{ml}$, interassay CV $<10 \%$.

\section{Dietary assessment}

Either the validated Italian or the English European Prospective Investigation into Cancer and Nutrition (EPIC) food frequency questionnaire (FFQ) [17] were used to evaluate dietary intake. A computer program, Nutrition Analysis of FFQ (NAF) [18] was developed by the Epidemiology and Prevention Unit, Fondazione IRCCS, Istituto Nazionale dei Tumori, Milan to convert questionnaire dietary data into frequencies of consumption and average daily quantities of foods, energy, folate and vitamin B6 intake. NAF was linked to either to the Mc Cance Food composition Tables (FCT) for UK data [19], or to the Italian FCT, for Italian data [20]. According to Recommended Dietary Allowance for adults [21], dietary folate intake of $400 \mu \mathrm{g} / \mathrm{day}$ and $200 \mu \mathrm{g} /$ day were considered as optimal and lowest recommended intake, correspondingly. From the 164 food items included in the EPIC-FFQ, food sources of folate were categorized in 15 major food groups as follows: "leafy vegetables", "broccoli \& root vegetables", "tomato \& other vegetables", "legumes", “citrus fruits", "other fruits", "fruit \& vegetable juices", "dried fruits", "pasta rice \& cereals", "potatoes \& bread", "breakfast cereals", "nuts \& seeds", "red meat \& products", "white meat \& egg", "fish" and "dairy". The use of vitamin supplements was evaluated in the UK population as a binary factor (yes or no). Data on their exact prescription was missing.

\section{Statistical analysis}


Normally distributed continuous variables are presented as mean $\pm \mathrm{SD}$, skewed as median $\left(1^{\text {st }}, 3^{\text {rd }}\right.$ quartiles $)$ and categorical variables as frequencies. Comparisons of continuous variables between two groups of study were performed using the independent Student's t-test, for the normally distributed variables and the MannWhitney test, for the skewed ones. Associations between categorical variables were tested using the Pearson's chi-square test.

Reduced rank regression (RRR) was used to derive dietary patterns (for Italy and UK) including major food sources of folate better describing a high dietary folate intake. RRR extracts linear functions of predictors (named factors) that explain as much response variation as possible [22]. RRR produces as many factors as there are dependent variables, which in our case is only one. The correlations between each extracted factor and foods are called factor loadings; we characterized the factor using the foods with an absolute factor loading greater than 0.20 . Each subject received, for each pattern, a factor score (RRR-score), calculated by summing the observed intake of the 15 food groups, each weighed by factor loadings [22]. An increase in this score represents increasing adherence to the factor-dietary pattern that better describes the high dietary folate intake.

Linear regression analysis was used separately for Italian and UK participants, to evaluate the association between dietary folate intake and food group consumption. Unadjusted models with main outcome dietary folate intake and independent factors each food group consumption was derived. Furthermore, in order to evaluate the percentage of the total variance of dietary folate intake that was explained by the food group consumption, multiple regression models including all food items were performed. Partial $\mathrm{R}^{2} \%$ conducted from these analyses indicated the aforementioned percentage. Using the same setting standardized $\beta$-coefficients was also produced to 
comparatively evaluate the effects of independed factors to the main outcome. They were reported as percent absolute values. The same regression analyses were performed to evaluate the association between dietary folate intake and other participant characteristics. Unadjusted and multi-adjusted linear regression analyses separated by either Country were also performed to evaluate the possible associations of folate serum levels with food group consumption, dietary folate intake and other characteristics. Because of the log-transformation of the dependent factor in each of all the aforementioned cases, the 1 unit increase in the independent factor caused the $\beta$-coefficient ( $\beta$-coef) to be equal with the log ratio of the dependent situations after and before the 1 unit increase. So the $e^{\beta \text {-coef }}$ should be equal to the ratio of the dependent situations after and before the 1 unit increase in the independent factor; results were presented as $\mathrm{e}^{\beta \text {-coef }}$, describing how many times 1 unit increase in the independent factor increased or decreased the dependent.

In all regression models normality of residuals, homoscedasticity, and multiple co-linearity were evaluated by plotting standardized residuals against the predicted values. All tested hypotheses were two-sided. P-value $<0.05$ was considered as statistically significant. STATA version 9 software was used for all calculations (STATA Corp., Texas, USA) except from RRR where SAS software (version 9.1.3 for Windows, Cary, NC: SAS Institute Inc. 2000-2004) was used.

\section{RESULTS}

\section{Dietary intake of folate in Italy and UK}

Figure la presents the distribution of dietary folate intake in men and women according to either country. Mean folate intake was higher in Italian men than women $(\beta$-coef $=29.4, p<0.001)$, while no gender difference was observed in UK $(\beta$-coef $=-7.6$, 
$\mathrm{p}=0.49$ ). In both genders, English people had greater dietary folate intake than Italians (P for country differences $<0.001$ ). The percentage of participants that reached the lowest recommended intake of $200 \mu \mathrm{g}$ /day was $83 \%$ of Italian and $96 \%$ of English subjects $(\mathrm{P}<0.001)$. However, only $11.3 \%(15.1 \%$ of men and $7.4 \%$ of women, $\mathrm{P}<0.001)$ of Italians exceeded the optimal recommended intake of $400 \mu \mathrm{g} / \mathrm{day}$ (Recommended Dietary Allowance for adults) against $45.1 \%$ of UK participants (44.9\% of men and $45.3 \%$ of women, $\mathrm{P}=0.93)(\mathrm{P}<0.001)$. Further analysis showed that in Italy, menopausal women had greater dietary folate intake $(362(273,455)$ $\mu \mathrm{g} / \mathrm{d})$ than pre-menopause women $(298(244,378) \mu \mathrm{g} / \mathrm{d}),(\mathrm{P}<0.001)$ while no significant difference was observed in the UK $(\mathrm{P}=0.18)$. The percentage of premenopausal women who reached the recommended levels of dietary folate intake $(\mathrm{RDA}=400 \mu \mathrm{g} / \mathrm{d})$ was only $8.1 \%$ in Italy but $41.4 \%$ in $\mathrm{UK}(\mathrm{P}<0.001)$.

Figure 2 presents the partials $\mathrm{R}^{2} \%$ calculated from multivariate analysis in Italian and UK participants. Total variance of dietary folate intake that was explained by food groups was $88.3 \%$ in Italy and $89.5 \%$ in UK. In Italians, potatoes and bread explained $61.5 \%$ of the folate intake variance, followed by leafy vegetables, fruit and vegetables, juices, pasta and rice and citrus fruit. In UK, at variance, broccoli and root vegetables explained more of the dietary intake folate variance (Partial $\mathrm{R}^{2} \%=59.1$ ) followed by breakfast cereals, potatoes and bread, dairy and leafy vegetables.

In Italy a factor-dietary pattern was derived which could explain $85.9 \%$ of the total variation of dietary folate intake and $15.7 \%$ of the total variation between food groups (Table 1). The "Italian dietary folate intake pattern" was described by high consumption of every different type of vegetables, legumes, potatoes and bread, noncitrus fruits and white meat. The results of RRR in UK also revealed a factor-dietary pattern explaining $82 \%$ of the total variation of dietary folate intake and the $14 \%$ of 
the total variation between food groups. The "UK dietary folate intake pattern" was described by high consumption of vegetables, non-citrus fruits, potatoes and bread, breakfast cereals and dairy.

\section{Folate serum levels in Italy and UK}

Figure $1 b$ presents the distribution of folate levels in men and women according to either country. In both genders, UK participants showed higher folate levels than Italians (P for country differences $<0.001$ ). This was also indicated by the percentage of participants who had a favourable folate status (serum levels $>6.62 \mathrm{ng} / \mathrm{ml}$ or $15 \mathrm{nmol} / \mathrm{L}$ ) Italy $29.0 \%$ and UK $75.5 \%(\mathrm{P}<0.001)$. Menopausal women had higher folate levels $(8.8(6.3,11.1) \mathrm{ng} / \mathrm{ml})$ than pre-menopause $(6.7(4.9,9.2)$ $\mathrm{ng} / \mathrm{ml}$ ). In addition, only $33.3 \%$ of Italian women at childbearing age had favourable folate status, while in UK they were $77.9 \%(\mathrm{P}<0.001)$.

Multivariate analyses showed that Italian participants who were classified in the higher quartile of folate levels had greater daily consumption of citrus fruits, leafy vegetables, and fish as compared with those in the lowest quartile (Table 2a). On the contrary, red meat, legumes and dairy were less consumed. The total percentage of variance of folate levels explained by food group consumption was $14.2 \%\left(\mathrm{R}^{2} \%\right.$ from multivariable model). The "Italian high folate dietary pattern" was also positively associated with serum folate levels in the multivariable model $(\mathrm{P}<0.001)$.

Italians who were classified in the highest quartiles of folate levels were older, more frequently women, with higher rate of high physical activity and lower rate of current smoking habits as well as lower energy intake ( $\mathrm{P}$ for all<0.05) (Table $2 b)$. According to both $\mathrm{R}^{2} \%$ and Istandardized $\beta$-coefl $\%$, the "Italian high folate dietary pattern" was the most important factor explaining folate levels variability. Moreover, 
energy intake accounted for a relatively high percentage of explained variability $\left(\mathrm{R}^{2} \%=3.7\right)$. On the contrary, physical activity was the less important factor.

UK participants with the highest folate levels had greater daily consumption of citrus fruit, breakfast cereals and fish and lower intake of red meat $(\mathrm{P}$ for all<0.05) (Table 3a). The total percentage of variance of folate levels explained by food group consumption was $16.3 \%\left(\mathrm{R}^{2} \%\right.$ from multivariable model).

UK participants with the highest folate levels showed higher adherence to the "English high folate dietary pattern" and a more frequent use of vitamin supplements $(\mathrm{P}$ for all<0.05) (Table $3 b)$. Moreover, they were less frequently smokers and had both lower energy intake and BMI. According to both $\mathrm{R}^{2} \%$ and Istandardized $\beta$-coefl $\%$ the "English high folate dietary pattern" was the most important factor in explaining folate level variability. In addition, the use of vitamin supplements $\left(\mathrm{R}^{2} \%=3.6\right)$ and tobacco $\left(\mathrm{R}^{2} \%=2.9\right)$ accounted for a relative high percentage of explained variability. In contrast, energy intake was the less important $\left(\mathrm{R}^{2} \%=1.1\right)$.

In both populations dietary folate intake was positively associated with folate levels after adjustments for confounders ( $\mathrm{P}$ for all<0.05). An increase of $100 \mu \mathrm{g} / \mathrm{day}$ in dietary folate intake was associated with $13.8 \%$ and $10.5 \%$ increase in folate levels in the Italian and UK population, respectively (for $100 \mu \mathrm{g} /$ day increase $\mathrm{e}^{\beta \text {-coef }}=1.138$ and $1.105, \mathrm{P}<0.001)$.

\section{DISCUSSION}

\section{Dietary folate intake in Italy and UK}

In this study, an inadequate dietary folate intake was observed in SouthernItalian subjects, while in S-W London people the folate status appeared to be better. 
Only $11.3 \%$ of Italians but $45.1 \%$ of English people exceed the recommended intake of $400 \mu \mathrm{g} /$ day, as an indication of a better but non-optimal dietary profile.

The average European intake (UK excluded) according to recent evidence from EPIC-study was $307 \mu \mathrm{g} / \mathrm{d}$ in men and $252 \mu \mathrm{g} / \mathrm{d}$ in women [23] while in a review of de Bree et al. the dietary folate intake did not exceed $320 \mu \mathrm{g} / \mathrm{d}$ [24]. EPIC data have also indicated that an English health- conscious group (mainly vegetarians or vegans) had markedly greater intake than other EU populations [23].

The percentage of women at childbearing age who did not reach the appropriate intake of folate was quite high in both Countries. Indeed, 91.9\% and $58.6 \%$ of Italian and UK women at childbearing age did not succeed to reach the intake of $400 \mu \mathrm{g} / \mathrm{d}$, which is quite safe at this reproductive age. In US after the fortification strategy of grain and cereal products, spina bifida and anencephaly rates were reduced by about $20 \%$ [25].

To understand possible factors responsible for the low intake of folate in the two populations we examined food sources of folate.

Potatoes and bread explained $61.5 \%$ of the total variance of dietary folate intake in Italians followed by leafy vegetables; while the corresponding food groups in UK were broccoli and root vegetables $(59.1 \%$ of total variance) followed by breakfast cereals. Parallel pattern analysis resulted in similar conclusions. The "Italian dietary folate intake pattern", which was characterized by high intake of vegetables, potatoes, bread, fruits, legumes and white meat described more precisely the high dietary folate intake. The corresponding "UK pattern" differed only in legumes and white meat consumption, which was replaced, by breakfast cereals and dairy. 
These findings are in agreement with previous European data that indicated vegetables, cereals and cereal products as the most important folate sources, both in Italy and UK [23].

In addition, both dietary patterns extracted by the present "a posteriori' dietary analysis included high consumption of food groups common with those included in the traditional Mediterranean diet, a pattern recently associated to a better folate nutritional adequacy [26].

\section{Folate serum status in Italy and UK}

As far as folate intake is concerned, folate serum levels were generally inadequate in the Italian population, while UK subjects showed a better profile. Again, women at childbearing age had lower folate serum levels than at menopause, probably because of a lower dietary intake.

A recent study by Dhonukshe-Rutten et al. [16] indicated that using the same cut off $(6.62 \mathrm{ng} / \mathrm{ml}$ or $15 \mathrm{nmol} / \mathrm{L})$, only in Germany, Sweden, UK and Spain folate serum levels seemed to be adequate. The three Italian studies included in that European survey reported an alarming situation. Similarly, a more recent analysis [27] indicated that only $22.5 \%$ of a Southern Italy population had adequate serum folate levels. On the contrary, results from US after the mandatory fortification strategy revealed a more adequate folate serum profile [28].

Possible reasons for such difference include dietary intake of folate and the fortification strategies that had been followed by national health policies. Dietary intake profile of UK subjects was more adequate than that of the Italians. In UK, public health promotion efforts have been made the last 2 decades recommending consumers to prefer fortified foods as a health protection choice. More recently 
(2007), the UK Food Standards Agency has approved the suggestion for food industries to fortify bread or flour with folate, however, there is still no decision for a mandatory fortification. [11].

Food group consumption in Italy and UK explained respectively $14.2 \%$ and $16.3 \%$ of serum folate variability. Similarly, the adherence to a high folate dietary pattern was positively associated with folate serum levels in both countries. Conversely, red meat, legumes and dairy, although containing folate, were inversely associated with folate serum levels. Tanking into account that the use of FFQ may not always allow to identify strong associations of dietary and serum folate, the intake of food rich in folate seems to result somehow in higher folate serum levels. Available data both from cross-sectional [29, 30] and diet-intervention studies [31, 32] suggest a positive association between folate status and dietary patterns characterized by high consumption of fruits, vegetables, and low consumption of red meat and dairy. Probably the increase in the latter consumption might be an indicator of an unhealthy dietary pattern with limited servings of fruits, vegetables and cereals. In addition bioavailability of folate in different food groups, that has not been yet fully understood [33], might explain the reported proportion of explained variability of serum folate levels by food intake.

UK participants with the highest folate levels showed a more frequent use of vitamin supplements. This could be in part an explanation for the better profile. However, data about exact prescription of vitamins was missing.

In both EU populations energy intake was positively associated with serum folate levels. Indeed, in the Italian population energy consumption explained 3.7\% of the total folate levels variability. Indeed, lower energy intake has been associated with better quality of diet through lower energy-dense and healthier foods choices [34]. 
This might lead to a better and well-absorbed folate intake. In addition, low energy reporting is usually associated to a healthier lifestyle [35] (e.g. lower smoking habits, greater physical activity), which, according to the present results, was also associated with higher folate levels.

Among non-dietary environmental factors, non-smoking was associated with better folate profile. In the study by Vardavas et al., in a healthy Greek population, non-smokers had $13 \%$ greater folate serum levels than smokers [36]. Similar findings were observed in a population of pregnant women where non-smokers had $22 \%$ higher levels of folate serum levels than smokers [37]. EPIC study bearded out the same conclusion [23]. The underlined biological mechanism of this association could be explained by the chemical components found in tobacco smoke which interact with folate in blood by transforming them into inactive compounds, reducing their active concentration in biological fluids and possibly altering the ability of the cell to store and metabolize them [38].

Physical activity was positively associated with folate serum levels in Italians, as already observed in Southern Italy, where more active individuals had significant greater folate serum levels as compared to inactive [27]. According to our knowledge there is no biological mechanism explaining the positive association of physical activity rate with serum folate levels. However, physical active populations seemed to use tobacco less frequent [39] while they had greater adherence to healthy dietary patterns including high quantities of fruits, vegetables and cereals [40]; factors that, in our study, were associated with higher folate levels.

\section{Limitations of this study}


Although the data reported here appear of high public health interest, this study has some limitations. First, the cross-sectional design of the IMMIDIET study does not enable determination of causality. Secondly, possible errors because of misreporting by participating subjects should be acknowledged. The use of FFQ as a dietary assessment method may not always allow identifying strong associations of intake and folate levels. In addition, the use of different food composition tables might insert possible error in the evaluation of folate intake in the $2 \mathrm{EU}$ populations. Generalization of the present conclusions to the total Italian or UK population should be avoided, since the recruitment of study participants, although randomly made from the general population, was limited to Abruzzo region and S-W London.

\section{Conclusions}

In this study, both inadequate dietary folate intake and serum levels were observed in Italian subjects, while in S-W London people the folate status appeared to be somewhat better. Differences between country in food group consumption as good sources of folate could in part explain this phenomenon. Non-smoking habit and physical activity were the two non-dietary, lifestyle characteristics positively associated with folate serum levels.

Folate is a potentially relevant factor in the prevention of a number of diseases. The evidence linking folate to NTD prevention must lead to the introduction of public health strategies to increase folate intake, in particular in Countries with evidence of low intake and low serum levels of folate. Different approaches should be used, such as pharmacological supplementation and/or mandatory or voluntary fortification of staple foods with folate, although they should never replace dietary improvement strategies. 
402

403 ACKNOWLEDGEMENTS

404 The study was supported by European Union grant no QLK1-2000-00100. 


\section{REFERENCES}

[1] Trichopoulou A, Costacou T, Bamia C, Trichopoulos D. Adherence to a Mediterranean diet and survival in a Greek population. N Engl J Med. 2003;348:2599-608.

[2] McNulty H, Pentieva K, Hoey L, Ward M. Homocysteine, B vitamins and CVD. Proc Nutr Soc. 2008; doi:10.1017/S0029665108007076.

[3] Refsum H, Ueland PM, Nygard O, Vollset SE. Homocysteine and cardiovascular disease. Annu Rev Med. 1998; 49: 31-62.

[4] Ciccarone E, Di Castelnuovo A, Assanelli D, Archetti S, Ruggeri G, Salcuni N, et al. Homocysteine levels are associated with the severity of peripheral arterial disease in Type 2 diabetic patients. J Thromb Haemost. 2003; 1: 2540-7.

[5] Clarke R, Halsey J, Lewington S, Lonn E, Armitage J, Manson JE, et al. Effects of lowering homocysteine levels with B vitamins on cardiovascular disease, cancer, and cause-specific mortality. Arch Intern Med. 2010; 170: 1622-31.

[6] Wang X, Qin X, Demirtas H, Li J, Mao G, Huo Y, et al. Efficacy of folic acid supplementation in stroke prevention: a meta-analysis. Lancet. 2007; 369: 187682.

[7] The Heart Outcomes Prevention Evaluation (HOPE) 2 Investigators. Homocysteine lowering with folic acid and B vitamins in vascular disease. N Engl J Med. 2006; 354: 1567-77.

[8] MRC Vitamin Study Research Group. Prevention of neural tube defects: results of the Medical Research Council Vitamin Study. Lancet. 1991; 338: 131-37.

[9] Grosse SD, Waitzman NJ, Romano PS, Mulinare J. Reevaluating the benefits of folic acid fortification in the United States: economic analysis, regulation, and public health. Am J Public Health. 2005; 95: 1917-22. 
[10] Smith AD, Kim YI, Refsum H. Is folic acid good for everyone? Am J Clin Nutr. 2008; 87: 517-33.

[11] Scientific Advisory Committee on Nutrition. Report: Folate and Disease Prevention. 2006. Available

at http://www.sacn.gov.uk/pdfs/folate_and_disease_prevention_report.pdf (last accessed 27 August 2013).

[12] Tucker KL, Selhub J, Wilson PW, Rosenberg IH. Dietary intake pattern relates to plasma folate and homocysteine concentrations in the Framingham Heart Study. J Nutr. 1996 Dec;126(12):3025-31.

[13] Iacoviello L, Arnout J, Buntinx F, Cappuccio FP, Dagnelie PC, de Lorgeril M, et al. Dietary habit profile in European communities with different risk of myocardial infarction: the impact of migration as a model of gene-environment interaction. The IMMIDIET Study. Nutr Metab Cardiovasc Dis. 2001; 11: 1226.

[14] Vohnout B, Arnout J, Krogh V, Donati MB, de Gaetano G, Iacoviello L; European Collaborative Group of the IMMIDIET Project. Association between MTHFR C677T genotype and circulating folate levels irrespective of folate intake: data from the IMMIDIET Project. Nutrition. 2011; 27: 1209-10. doi: 10.1016/j.nut.2011.07.008.

[15] Cappuccio FP, Strazzullo P, Farinaro E Trevisan M. Uric acid metabolism and tubular sodium handling: Results from a population-based study. J Am Med Assoc. 1993; 270: 354-359.

[16] Dhonukshe-Rutten RA, de Vries JH, de Bree A, van der Put N, van Staveren WA, de Groot LC. Dietary intake and status of folate and vitamin B12 and their 

association with homocysteine and cardiovascular disease in European populations. Eur J Clin Nutr. 2009; 63: 18-30.

[17] Slimani N, Kaaks R, Ferrari P, Casagrande C, Clavel-Chapelon F, Lotze G, et al. European Prospective Investigation into Cancer and Nutrition (EPIC) calibration study: rationale, design and population characteristics. Public Health Nutr. 2002; 5: 1125-45.

[18] Pala V, Sieri S, Palli D, Salvini S, Berrino F, Bellegotti M, et al. Diet in the Italian EPIC cohorts: presentation of data and methodological issues. Tumori. 2003; 89: 594-607.

[19] Mc Cance RA, Widdowson EM. The composition of foods. 5th ed. Cambridge, United Kingdom: The Royal Society of Chemistry and Ministry of Agriculture, Fisheries and Food; 1991.

[20] Salvini S, Parpinel M, Gnagnarella P, Maisonnneuve P, Turrini A. Banca Dati di Composizione degli Alimenti per Studi Epidemiologici in Italia. Milan, Italy: Istituto Europeo di Oncologia; 1998.

[21] Institute of Medicine. Food and Nutrition Board (1998). Dietary Reference Intakes: Thiamin, Riboflavin, Niacin, Vitamin B6, Folate, Vitamin B12, Pantothenic Acid, Biotin, and Choline. Washington, DC, National Academy Press.

[22] K. Hoffman, M.B. Schulze, A. Schienkiewitz, U. Nöthlings, H. Boeing. Application of a new statistical method to derive dietary patterns in nutrition epidemiology. Am J Epidemiol. 2004; 159: 935-44.

[23] Park JY, Nicolas G, Freisling H, Biessy C, Scalbert A, Romieu I, et al. Comparison of standardised dietary folate intake across ten countries 
participating in the European Prospective Investigation into Cancer and Nutrition. Br J Nutr. 2012; 108: 552-69.

[24] de Bree A, van Dusseldorp M, Brouwer IA, van het Hof KH, SteegersTheunissen RP. Folate intake in Europe: recommended, actual and desired intake. Eur J Clin Nutr. 1997; 51: 643-60.

[25] Choumenkovitch SF, Selhub J, Wilson PW, Rader JI, Rosenberg IH, Jacques PF. dietary folate intake from fortification in United States exceeds predictions. J Nutr. 2002; 132: 2792-8.

[26] Serra-Majem L, Bes-Rastrollo M, Román-Viñas B, Pfrimer K, Sánchez-Villegas A, Martínez-González MA. Dietary patterns and nutritional adequacy in a Mediterranean country. $\mathrm{Br}$ J Nutr. 2009; 101: S21-8. doi: $10.1017 / \mathrm{S} 0007114509990559$.

[27] Zappacosta B, Persichilli S, Iacoviello L, Di Castelnuovo A, Graziano M, Gervasoni J, et al. Folate, vitamin B12 and homocysteine status in an Italian blood donor population. Nutr Metab Cardiovasc Dis. 2011 Dec 30. [Epub ahead of print].

[28] Pfeiffer CM, Johnson CL, Jain RB, Yetley EA, Picciano MF, Rader JI, et al. Trends in blood folate and vitamin B-12 concentrations in the United States, 1988-2004. Am J Clin Nutr. 2007; 86: 718-27.

[29] Tucker KL, Selhub J, Wilson PW, Rosenberg IH. Dietary intake pattern related to plasma folate and homocysteine concentrations in the Framingham Heart Study. J Nutr. 1996; 126: 3025-31.

[30] Kerver JM, Yang EJ, Bianchi L, Song WO. Dietary patterns associated with risk factors for cardiovascular disease in healthy US adults. Am J Clin Nutr. 2003; 78: $1103-10$. 
[31] Brouwer IA, van Dusseldorp W, West CE, Meyboom S, Thomas CMG, Duran $\mathrm{M}$, van het Hof $\mathrm{KH}$, et al. Dietary folate from vegetables and citrus fruit decreased plasma homocysteine concentrations in humans in a dietary controlled trial. J Nutr. 1999; 129: 1135-9.

[32] Kiefer I, Prock P, Lawrence C, Wise J, Bieger W, Bayer P, et al. Supplementation with mixed fruit and vegetable juice concentrates increased serum antioxidants and folate in healthy adults. J Am Coll Nutr. 2004; 23: $205-$ 11.

[33] Ohrvik VE, Witthoft CM. Human folate bioavailability. Nutrients. 2011; 3: 47590.

[34] de Oliveira MC, Sichieri R, Venturim Mozzer R. A low-energy-dense diet adding fruit reduces weight and energy intake in women. Appetite. 2008; 51: 291-5. doi: 10.1016/j.appet.2008.03.001.

[35] Johansson L, Solvoll K, Bjørneboe GE, Drevon CA. Under- and overreporting of energy intake related to weight status and lifestyle in a nationwide sample. Am J Clin Nutr. 1998; 68: 266-74.

[36] Vardavas CI, Linardakis MK, Hatzis CM, Malliaraki N, Saris WH, Kafatos AG. Smoking status in relation to serum folate and dietary vitamin intake. Tob Induc Dis. 2008; doi:10.1186/1617-9625-4-8.

[37] McDonald SD, Perkins SL, Jodouin CA, Walker MC. Folate levels in pregnant women who smoke: an important gene/environment interaction. Am J Obstet Gynecol. 2002; 187: 620-5.

[38] Northrop-Clewes CA, Thurnham DI. Monitoring micronutrients in cigarette smokers. Clinica Chima Acta. 2000; 377: 14-38. 
527 [39] Kaczynski AT, Manske SR, Mannell RC, Grewal K. Smoking and physical 528 activity: a systematic review. Am J Health Behav. 2008; 32: 93-110.

529 [40] Kavouras SA, Panagiotakos DB, Pitsavos C, Chrysohoou C, Arnaoutis G, 530 Skoumas Y, et al. Physical Activity and Adherence to Mediterranean Diet Increase Total Antioxidant Capacity: The ATTICA Study. Cardiol Res Pract. 2010; 2011: 248626. doi: 10.4061/2011/248626. 
533

534

535

536

537

538

539

540

541

542

543

\section{[41] LEGEND TO THE FIGURES}

Figure 1 Distribution of dietary folate intake and folate serum levels in men and women participants according to country of residence: (a) Italy, (b) UK. Daily folate intake $(\mu \mathrm{g})$ by country and gender: median $\left(1^{\text {st }}, 3^{\text {rd }}\right.$ quartile $)$ Italy, men: 284 (235, 344); Italy, women: $266(220,317)$; UK, men: 385 (312, 469); UK women: $384(316,476)$

Folate serum levels $(\mathrm{ng} / \mathrm{ml})$ by country and gender: median $\left(1^{\text {st }}, 3^{\text {rd }}\right.$ quartile $)$ Italy, men: $4.9(3.9,6.3)$; Italy, women: $5.9(4.4,7.3)$

UK, men: $8.1(6.3,10.5)$; UK women: $9.3(6.8,11.5)$

Figure 2 Contribution of food group consumption to dietary folate intake in Italy \& UK 
Appendices: European Collaborative Group of the IMMIDIET Project

Project Co-ordinator: Licia Iacoviello ${ }^{\mathrm{a}}$

Scientific Committee: Jef Arnout, ${ }^{\mathrm{c}}$ Frank Buntinx, ${ }^{\mathrm{d}}$ Francesco P. Cappuccio, ${ }^{\mathrm{e}}$ Pieter C. Dagnelie, ${ }^{\mathrm{f}}$ Maria Benedetta Donati, ${ }^{\mathrm{a}}$ Michel de Lorgeril,${ }^{\mathrm{g}}$ Vittorio Krogh, ${ }^{\mathrm{h}}$ Alfonso $\operatorname{Siani}^{\mathrm{i}}$

Co-ordinating secretariat: Carla Dirckx ${ }^{\mathrm{c}, \mathrm{d}}$

Data management and statistics: Augusto Di Castelnuovo ${ }^{\mathrm{a}}$

Dietary assessment and analysis: Martien van Dongen ${ }^{\mathrm{f}}$

Communication and dissemination: Americo Bonanni ${ }^{\mathrm{a}}$

Recruitment: Carla Dirckx, ${ }^{\mathrm{c}, \mathrm{d}}$ Pit Rink, ${ }^{\mathrm{e}}$ Branislav Vohnout, ${ }^{\mathrm{b}}$ Francesco Zito ${ }^{\mathrm{b}}$

External advisory committee: Mario Mancini, Napoli, Italy; Antonia Trichopoulou, Athens, Greece

The IMMIDIET group, collaborative centres and associated investigators (2012)

a. IRCCS Istituto Neurologico Mediterraneo Neuromed, Pozzilli, Isernia, Italy (Licia Iacoviello, Mari Benedetta Donati, Giovanni de Gaetano Amalia De Curtis, Augusto Di Castelnuovo, Americo Bonanni)

b. Fondazione di Ricerca e Cura "Giovanni Paolo II", Catholic University, Campobasso, Italy (Francesco Zito, Branislav Vohnout, Marco Olivieri, Agnieszka Pampuch)

c. Centre for Molecular and Vascular Biology, Katholieke Universiteit Leuven, Leuven, Belgium (Jef Arnout, Carla Dirckx, Ward Achten) 
d. Department of General Practice, Katholieke Universiteit Leuven, Leuven, Belgium (Frank Buntinx, Carla Dirckx, Jan Heyrman)

e. Clinical Sciences Research Institute, Warwick Medical School, Coventry, United Kingdom (Francesco P. Cappuccio, Michelle A Miller); Division of Community Health Sciences, St George's, University of London, United Kingdom (Pit Rink, Sally C Dean, Clare Harper)

f. Department of Epidemiology, CAPHRI, Subdivision of Nutritional and Molecular Epidemiology, Maastricht University, Maastricht, The Netherlands (Pieter Dagnelie, Martien van Dongen, Dirk Lemaître)

g. Nutrition, Vieillissement et Maladies Cardiovasculaires (NVMCV), UFR de Médecine, Domaine de la Merci, 38056 La Tronche, France (Michel de Lorgeril)

h. Nutritional Epidemiology Unit, National Cancer Institute, Milan, Italy (Vittorio Krogh, Sabrina Sieri, Manuela Bellegotti, Daniela Del Sette Cerulli)

i. Unit of Epidemiogy \& Population Genetics, Institute of Food Sciences CNR, Avellino, Italy (Alfonso Siani, Gianvincenzo Barba, Paola Russo, Antonella Venezia) 
Table 1. Results from reduced rank regression analyses that evaluated the dietary pattern, which is associated with folate dietary intake of Italian and English IMMIDIET population.

\begin{tabular}{rrr} 
& \multicolumn{2}{c}{ Factor loadings $^{\text {a }}$} \\
\cline { 2 - 3 } Food groups (g/day) & Italy $(\mathrm{n}=542)$ & $\mathrm{UK}(\mathrm{n}=526)$ \\
\cline { 2 - 3 } Leafy vegetables & 39.2 & 30.4 \\
Broccoli \& root vegetables & 28.6 & 43.9 \\
Tomato \& other vegetables & 30.2 & 37.1 \\
Legumes & 29.3 & \\
Citrus fruits & & 23.4 \\
Other fruits & 32.7 & \\
Dried fruits & & \\
Fruit \& vegetable juices & & 28.9 \\
Pasta rice \& cereals & & 34.6 \\
Potatoes \& bread & 37.6 & \\
Breakfast cereals & & \\
Nuts \& seeds & & 26.7 \\
Red meat \& products & & \\
White meat \& egg & 28.0 & \\
Fish & & \\
Dairy & &
\end{tabular}

${ }^{\mathrm{a}}$ Factor loadings lower than 20.0 was not presented for simplicity. 


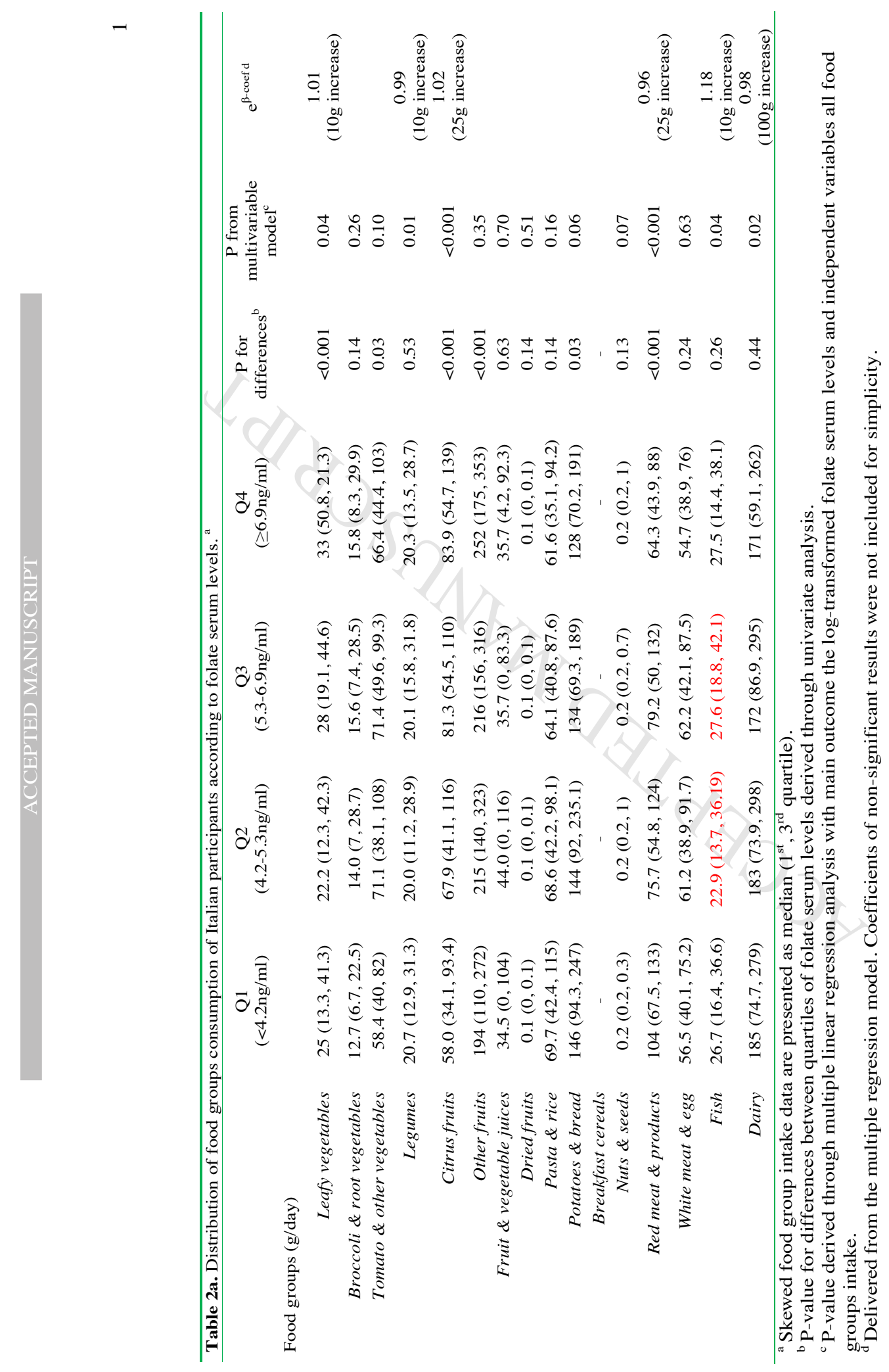




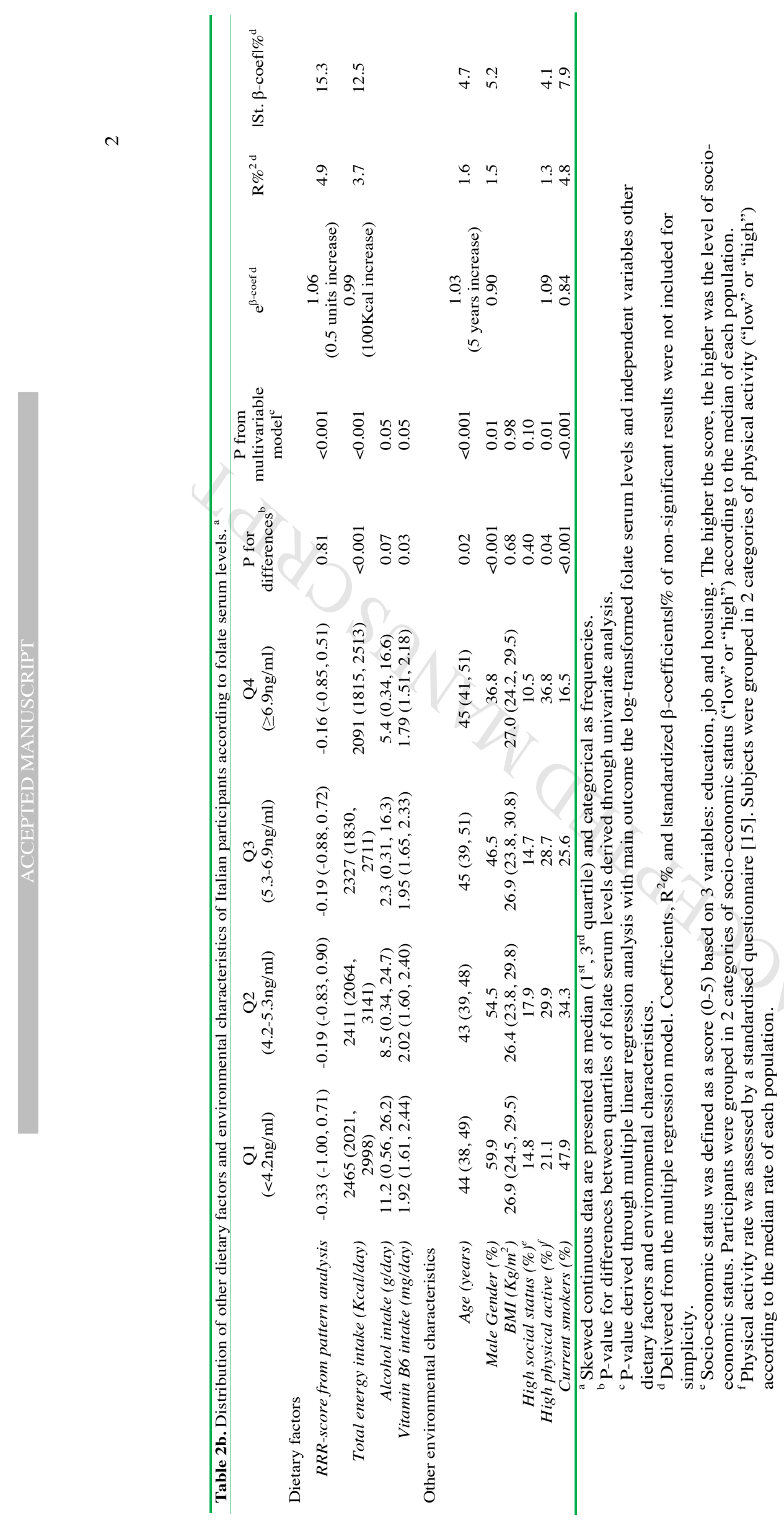




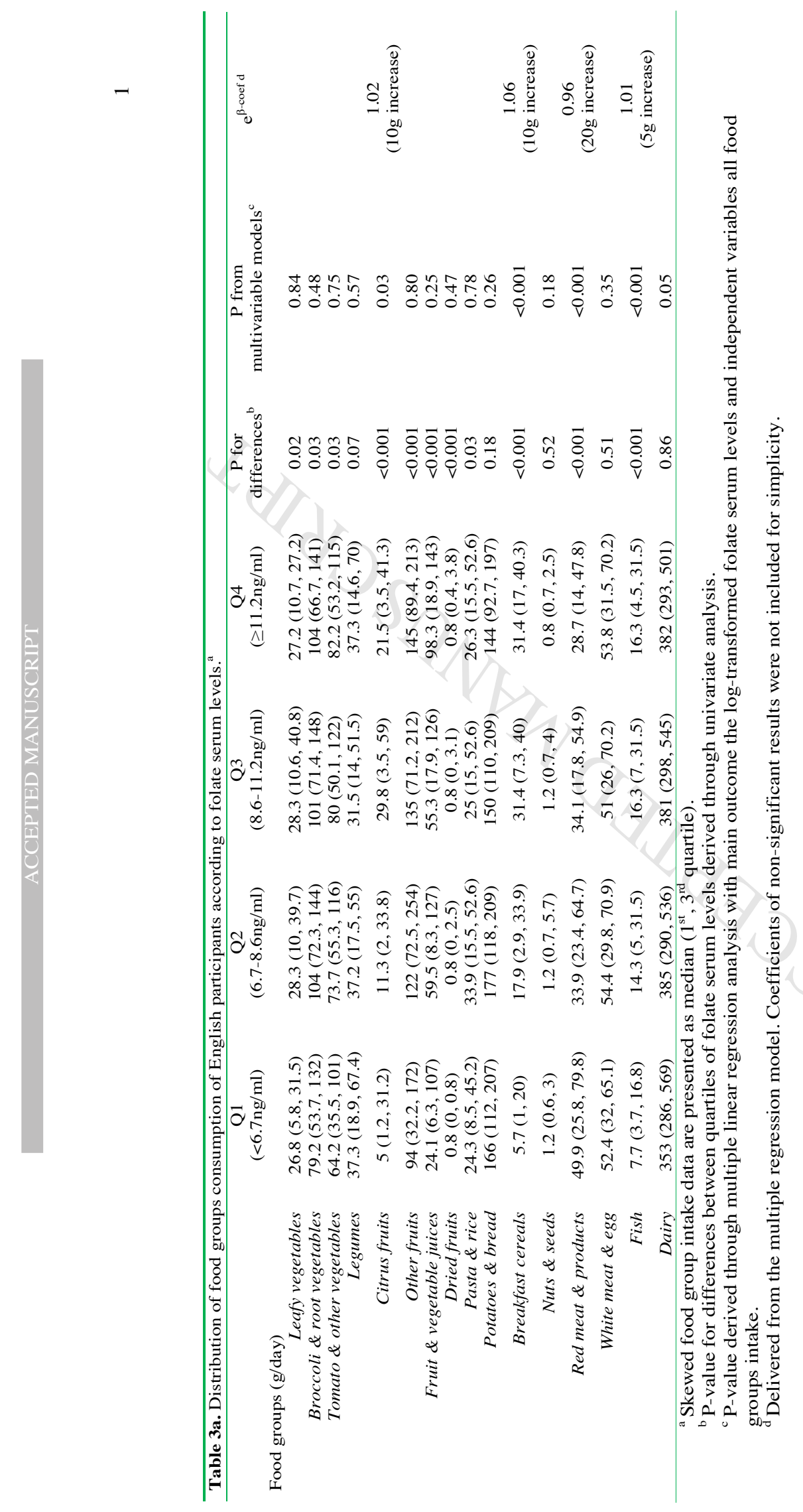




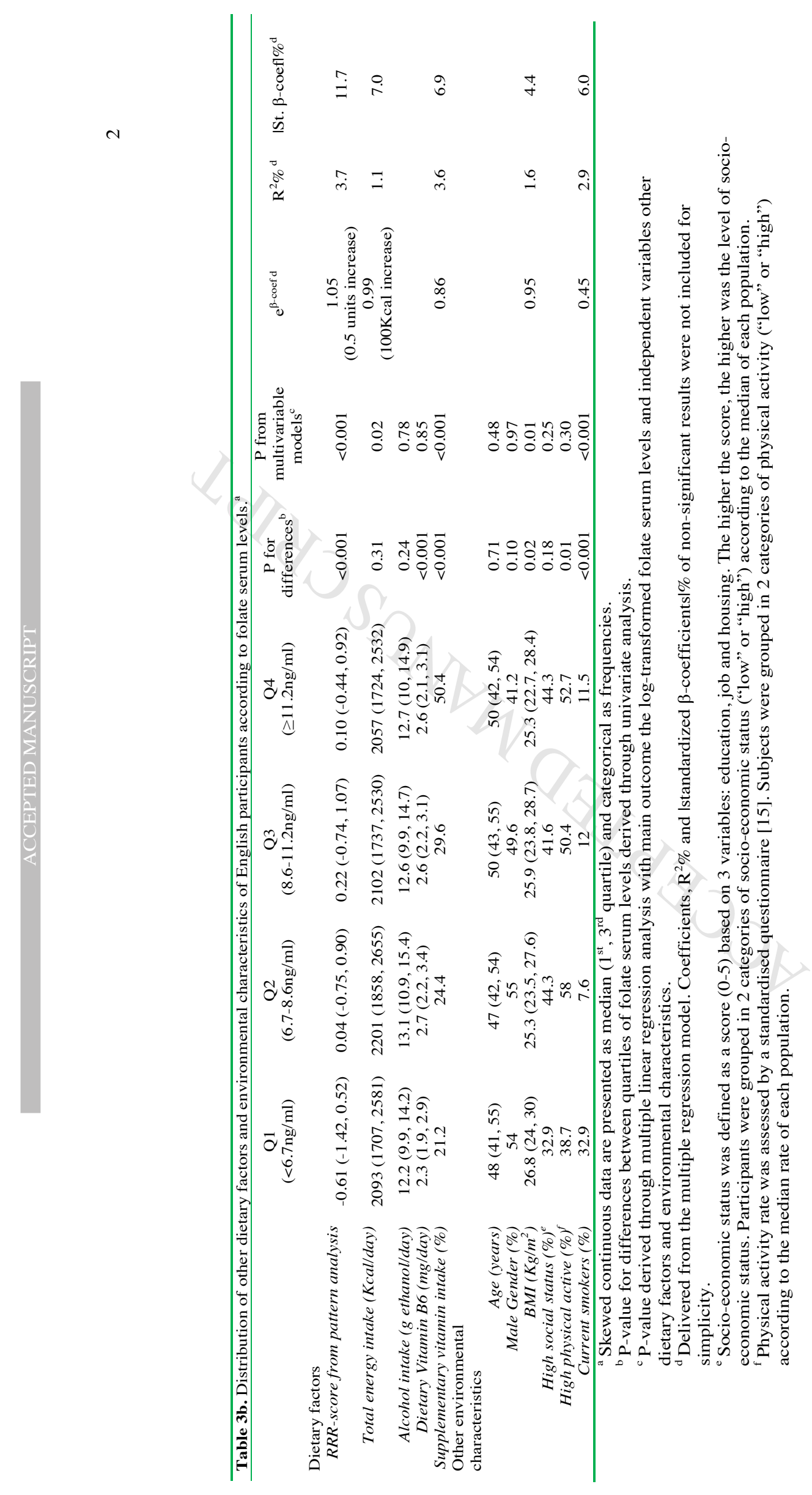



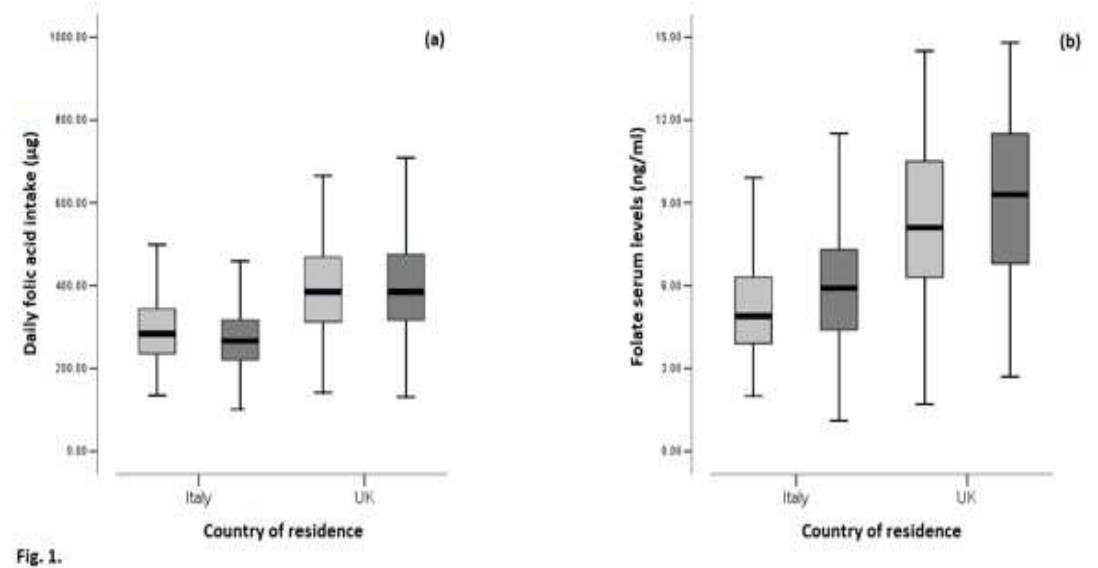

Fig. 1. 


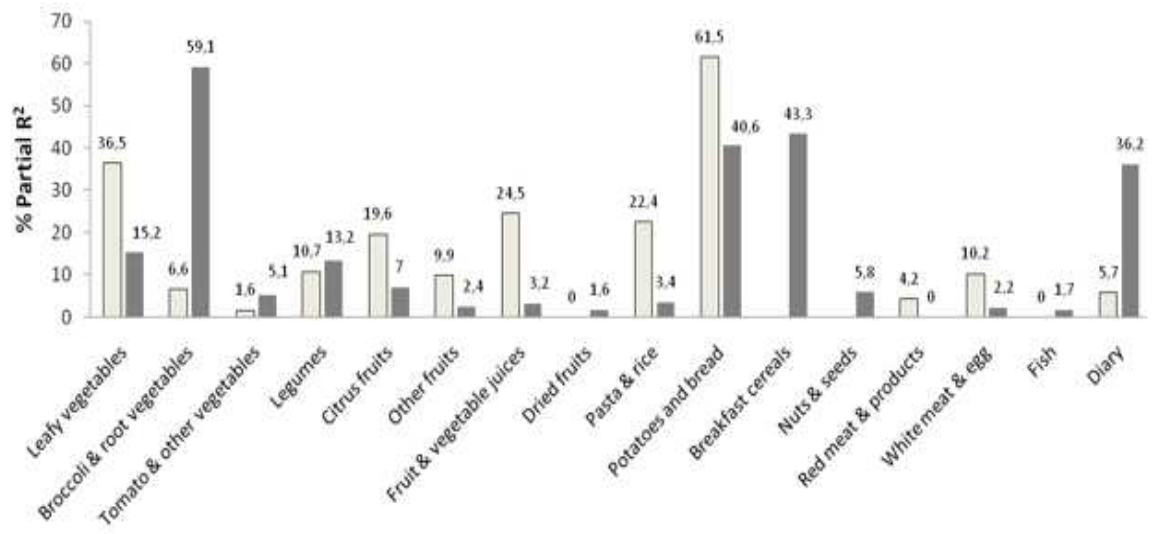

Fig. 2. 
Chapter 6.

Homocysteine lowering by folate-rich diet or pharmacological supplementations in subjects with moderate hyperhomocysteinemia

Zappacosta B, Mastroiacovo P, Persichilli S, Pounis G, Ruggeri S, Minucci A, Carnovale E, Andria G, Ricci R, Scala I, Genovese O, Turrini A, Mistura L, Giardina B, lacoviello L. Homocysteine lowering by folate-rich diet or pharmacological supplementations in subjects with moderate hyperhomocysteinemia. Nutrients 2013; 5: 1531-43. doi: $10.3390 /$ nu5051531. 


\title{
nutrients
}

ISSN 2072-6643

www.mdpi.com/journal/nutrients

Article

\section{Homocysteine Lowering by Folate-Rich Diet or Pharmacological Supplementations in Subjects with Moderate Hyperhomocysteinemia}

\author{
Bruno Zappacosta $^{1, *}$, Pierpaolo Mastroiacovo ${ }^{2}$, Silvia Persichilli ${ }^{3}$, George Pounis ${ }^{4}$, \\ Stefania Ruggeri ${ }^{5}$, Angelo Minucci ${ }^{3}$, Emilia Carnovale ${ }^{5}$, Generoso Andria ${ }^{6}$, Roberta Ricci ${ }^{7}$, \\ Iris Scala ${ }^{6}$, Orazio Genovese ${ }^{7}$, Aida Turrini ${ }^{5}$, Lorenza Mistura ${ }^{5}$, Bruno Giardina ${ }^{3}$ \\ and Licia Iacoviello 4
}

1 Department of Laboratory, Foundation of Research and Care, "John Paul II", Catholic University of Sacred Heart, Campobasso 86100, Italy

2 ICBD, Alessandra Lisi International Centre on Birth Defects and Prematurity, Rome 00100, Italy; E-Mail: icbd@icbd.org

3 Department of Laboratory Medicine, Policlinico A. Gemelli, Catholic University of Sacred Heart, Rome 00168, Italy; E-Mails: spersichilli@rm.unicatt.it (S.P.); aminucci@rm.unicatt.it (A.M.); bgiardina@rm.unicatt.it (B.G.)

4 Research Laboratories, Foundation of Research and Care, "John Paul II", Catholic University of Sacred Heart, Campobasso 86100, Italy; E-Mails: gdpounis@moli-sani.org (G.P.); licia.iacoviellooli-sani.org (L.I.)

5 National Institute of Research for Foods and Nutrition (Istituto Nazionale di Ricerca per gli Alimenti e la Nutrizione), Rome 00178, Italy; E-Mails: ruggeri@inran.it (S.R.); carnovale@inran.it (E.C.); turrini@inran.it (A.T.); mistura@inran.it (L.M.)

6 Department of Pediatrics, Federico II University, Neaples 81100, Italy; E-Mails: andria@unina.it (G.A.); scala@unina.it (I.S.)

7 Department of Pediatrics, Catholic University of Sacred Heart, Rome 00168, Italy; E-Mails: rricci@rm.unicatt.it (R.R.); ogenovese@rm.unicatt.it (O.G.)

* Author to whom correspondence should be addressed; E-Mail: bzappacosta@rm.unicatt.it; Tel.: +39-0874-312-331; Fax: +39-0874-312-388.

Received: 17 January 2013; in revised form: 22 March 2013 / Accepted: 10 April 2013 /

Published: 8 May 2013

\begin{abstract}
Background/Objectives: To compare the efficacy of a diet rich in natural folate and of two different folic acid supplementation protocols in subjects with "moderate" hyperhomocysteinemia, also taking into account C677T polymorphism of
\end{abstract}


5,10-methylenetetrahydrofolate reductase (MTHFR) gene. Subjects/Methods: We performed a 13 week open, randomized, double blind clinical trial on 149 free living persons with mild hyperhomocyteinemia, with daily $200 \mu \mathrm{g}$ from a natural folate-rich diet, $200 \mu \mathrm{g}$ [6S]5-methyltetrahydrofolate (5-MTHF), $200 \mu \mathrm{g}$ folic acid or placebo. Participants were stratified according to their MTHFR genotype. Results: Homocysteine (Hcy) levels were reduced after folate enriched diet, 5-MTHF or folic acid supplementation respectively by $20.1 \%(p<0.002), 19.4 \%(p<0.001)$ and $21.9 \%(p<0.001)$, as compared to baseline levels and significantly as compared to placebo $(p<0.001, p<0.002$ and $p<0.001$, respectively for enriched diet, 5-MTHF and folic acid). After this enriched diet and the folic acid supplementation, Hcy in both genotype groups decreased approximately to the same level, with higher percentage decreases observed for the TT group because of their higher pre-treatment value. Similar results were not seen by genotype for 5-MTHF. A significant increase in RBC folate concentration was observed after folic acid and natural folate-rich food supplementations, as compared to placebo. Conclusions: Supplementation with natural folate-rich foods, folic acid and 5-MTHF reached a similar reduction in Hcy concentrations.

Keywords: folic acid; 5-MTHF; homocysteine; folate-rich diet; MTHFR genotype; clinical trial

\section{Introduction}

Several large-scale epidemiological studies, mainly retrospective ones, have lead, during the last three decades, to the appreciation of the sulfur-containing aminoacid homocysteine (Hcy) as an independent risk factor for cardiovascular disease, mainly coronary heart disease and stroke, although its causal effect has been recently challenged by some B-vitamins Hcy-lowering trials [1], Hcy has been involved in the occurrence of other disorders such as cognitive disease $[2,3]$ cancer $[4,5]$ and, overall, adverse outcome of pregnancy and congenital defects $[6,7]$.

Plasma levels of Hcy are closely dependent on nutritional and genetic factors: the B-vitamins folate, vitamin B12 and vitamin B6 are required for Hcy metabolism; suboptimal folate status, together with the other B-vitamins deficiency, is commonly associated with elevated plasma Hcy, as a consequence of the role that folate plays in the one-carbon metabolism. Among the main determinants of Hcy levels, especially in the presence of low folate concentrations, we have to mention some genetic polymorphisms, mainly the C677T of the enzyme 5,10-methylenetetrahydrofolate reductase (MTHFR): the TT genotype is frequently associated with elevated plasma levels of Hcy, due to a reduced production of 5-methyltetrahydrofolate (5-MTHF).

With regard to the present knowledge, the best strategy to ensure an optimal folate status is still uncertain; this is due to variable dietary habits, variable folate intake with diet, variable prevalence of mutated allele of the MTHFR gene in the different populations studied and the different bioavailability of natural folate and synthetic folic acid [8]. Recently, Dhonukshe-Rutten et al. [9] have shown how, 
in several European countries, both the intake and the B-vitamins status are inadequate and the Hcy concentration higher than ideal.

In Italy, a country in which a mandatory food fortification with folic acid has not been implemented and the use of fortified food is quite limited, the wide availability of folate-rich foods could suggest as the better strategy a folate-rich diet compared to daily intake of synthetic products as capsules. Furthermore, due to the relative high frequency of polymorphisms in MTHFR gene in the Italian population, it could be useful to verify the real efficacy of a greater folate intake in homozygote and heterozygote subjects for the mutated MTHFR gene, in reducing plasma levels of homocysteine.

Aim of this study was to compare the efficacy of a diet particularly rich in natural folate in relation to that of two different protocols of folic acid pharmacological supplementation, in subjects with "moderate" hyperhomocysteinemia, also taking into account the C677T polymorphism of the gene codifying for MTHFR enzyme.

\section{Patients and Methods}

\subsection{Study Population}

From September to November 2000, about 2000 subjects were approached among the staff and student populations of the "Agostino Gemelli" University Hospital of the Catholic University, School of Medicine, and the staff of the National Institute for Research on Health and Nutrition (INRAN) in Rome, Italy.

From December 2000 to February 2001, suitable volunteers $(n=1240,481$ men and 759 women, aged 18-60 years) were screened for total plasma Hcy levels.

Exclusion criteria were current use of B-vitamins supplements, presence of any acute or chronic disease, pregnancy or planned pregnancy and use of medications interfering with folate metabolism. The study was approved by the Ethical Committee of the Catholic University; written informed consent was obtained from all subjects.

Subjects with Hcy levels between the $75^{\circ}$ (Hcy $=10.0 \mu \mathrm{mol} / \mathrm{L}$ ) and the $97^{\circ}$ centile (Hcy $=22.2 \mu \mathrm{mol} / \mathrm{L}, n=273$ ) were invited to participate into the trial. The same group of subjects was studied for the MTHFR C677T polymorphism. One hundred fifty eight agreed to participate and 149 completed the study.

There were no significant differences in Hcy levels between the 149 subjects who completed the study and the 124 who did not accept $(n=115)$ or withdrew early $(n=9)(11.8 \mu \mathrm{mol} / \mathrm{L}, 10.7-13.6 v s$. $11.7 \mu \mathrm{mol} / \mathrm{L}, 10.7-13.6, p<0.7$; median and interquartile range) and in the main characteristics including MTHFR genotypes. However, the number of vegetable eaters was higher in the participant group $(58.0 \%$ vs. $44.2 \%, p<0.05)$.

\subsection{Dietary Assessment}

Food consumption data were collected at individual level every 10 days using the estimated one-day food record method with a semi-structured diary. All foods and drinks consumed, both at and outside home, were recorded by each participant using household measures and estimating portion sizes according to detailed guidance notes and photographs atlas [10]. The USDA food 
composition database was used to provide the folate content of foods (USDA Nutrient database for standard reference).

Since our study was planned before the advent of dietary folate equivalents (DFEs), a concept introduced to account for the varying bioavailability of folates from different sources [11], target intakes and analyses are presented in the original units ( $\mu \mathrm{g} /$ day), while estimated DFEs were presented in parentheses in the text.

\subsection{Study Design}

The study was a 13 week open, randomized, double blind clinical trial. For the intervention study, the following folate enrichment strategies were used:

- habitual diet (usually containing about $220 \mu \mathrm{g}$ of natural folate) $+200 \mu \mathrm{g}$ from natural folate-rich diet, daily; subjects were advised to consume their habitual diet plus additional natural folate-rich foods to achieve an additional intake of $200 \mu \mathrm{g} /$ day of folate; this was obtained throughout a "score diet of at least 10 points" (1 point $=20 \mu \mathrm{g})$;

- habitual diet + [6S]5-methyltetrahydrofolate $200 \mu \mathrm{g}$ (340 DFEs), daily;

- habitual diet + folic acid $200 \mu \mathrm{g},(340$ DFEs) daily;

- habitual diet + placebo.

The nutritional intervention was open, while the pill supplements were double-blinded.

Participants were also stratified according to their MTHFR genotype (42 subjects for each genotype) before randomization to ensure the same distribution of CC, CT and TT genotype in each group.

\subsection{Supplements}

Folic acid and [6S]5-MTHF capsules were obtained from Merck Eprova AG (Schaffhausen, Switzerland) as hard gelatin capsules containing $208 \mu \mathrm{g}(454 \mathrm{nmol})$ for the [6S]5-MTHF moiety), or $200 \mu \mathrm{g}(454 \mathrm{nmol})$ folic (monoglutamic) acid. The randomization code of the supplements was kept at the Institute of Food Reseach, Norwich, UK (Dr. PM Finglas).

The subjects were counseled in how to enrich the diet in order to achieve $400 \mu \mathrm{g}$ of daily folate intake, by a booklet showing the food folate content both for $100 \mathrm{~g}$ and per portion and suggesting the possible food combinations.

Compliance concerning the diet and the supplements was assessed by counting the pills still remaining, by phone interviews (at 4, 8 and 13 weeks).

All participants were advised do not use any other folate or vitamin supplements during the study.

Total plasma Hcy, RBC folate, vitamins B6 and B12 were determined at the beginning and at the end of the trial.

\subsection{Specimen Collection and Biochemical Measurements}

Blood samples were obtained between 08:00 AM and 10:00 AM after an overnight fast.

Serum sample was used for creatinine measurement on an automated chemistry analyzer (Roche Hitachi 917); hematocrit and red cell count were measured in tripotassium ethylendiaminetetraacetic acid (K3 EDTA) (Vacuette, Greiner Bio-One GmbH, Kremsmünster, Austria) on the Advia 120 
hematology analyzer (Bayer, Terrytown, NY, USA). These samples were analyzed within two hours from collection; internal quality control samples were daily processed with sample analysis.

For total Hcy assay, whole blood samples were collected on ice-cooled tubes containing K3 EDTA and centrifuged within $30 \mathrm{~min}$ at $2000 \times \mathrm{g}$ for $10 \mathrm{~min}$ at $4{ }^{\circ} \mathrm{C}$; plasma aliquots were frozen at $-80{ }^{\circ} \mathrm{C}$ until assayed. Hcy was assayed according to the HPLC method of Araki and Sako [12] with slight modifications, as reported elsewhere [13]. Intraassay and interassay coefficients of variation (CVs) were $<3.1 \%$ and $<4.5 \%$ respectively.

Whole blood samples, collected into K3 EDTA anticoagulated tubes, were used for RBC folate assay by using a microbiological assay according to Wright et al. [14]. The coefficient of variation of the assay was between $11.2 \%$ and $15.0 \%$. Concentration of $\mathrm{RBC}$ folate $\geq 305 \mathrm{nmol} \cdot \mathrm{L}^{-1}$ was considered adequate, according to the Institute of Medicine [11].

Vitamine B6 was measured by HPLC method with fluorimetric detection by using a commercially available kit (Chromsystems Instruments and Chemicals GmbH, Munchen, Germany), according to the manufacturer's instructions; intra and inter-assay coefficients of variation (CV) were $<4.0 \%$ and $<7.0 \%$ respectively. Vitamin B12 was measured by using an immunoassay on the Abbott AxSYM analyzer (Abbott Laboratories, Abbott Park, IL, USA); the total CV was $<10.0 \%$.

Blood collected on filter paper was directly used for PCR amplification for the study of the C677T mutation of the MTHFR gene at the Department of Pediatrics, Federico II University, Napoli, Italy. Half of the blood spot was cut and submerged in $130 \mu \mathrm{L}$ of distilled water, covered with a drop of paraffin oil, and heated at $96{ }^{\circ} \mathrm{C}$ for at least half an hour. At the end of this time, $25 \mu \mathrm{L}$ of the PCR amplification mix were added directly to the template ( $25 \mu \mathrm{L}$ of lysate). DNA amplification and restriction analysis by HinfI were carried out as previously described [15].

\subsection{Statistical Analysis}

Changes in RBC folate and Hcy concentrations between baseline and the follow-up visits were the primary variables of interest. Since their values were not normally distributed, median values with interquartile ranges (25th-75th percentiles) or geometric means and (95\% Confidence Intervals) were displayed. Changes in Hcy concentrations from baseline to follow-up were calculated by analysis of variance for non-parametric analysis by Kruskal-Wallis test. Differences in pre-treatment Hcy levels among MTHFR-polymorphism groups were derived through Mann-Whitney test. To calculate the odd to reach a good response (Hcy levels $<25 \%$ ) after enriched diet, 5-MTHF or folic acid, as compared to placebo, multiple logistic regression analysis, adjusted for sex, BMI, MTHFR genotypes, RBC folate, vitamin B6 and vitamin B12, was performed. Results were considered significant at $p<0.05$. (Statistical analyses were done by using Stata software, version 10.0.)

\section{Results}

The demographic characteristics of the study population and the variables measured at baseline are shown in Tables 1 and 2 . The four treatment groups did not differ significantly for age, sex, body mass index, social status, prevalence of smokers or physically actives and frequency of vegetarians, or wine or coffee drinkers. Serum levels of creatinine and hematocrit were similar across the groups. No 
significant differences among groups were observed in concentrations of total Hcy and vitamin cofactors and in the distribution of MTHFR genotypes.

Table 1. Distribution of demographic, anthropometric, life-habits and genetic characteristics of participants according to treatment group ${ }^{1}$.

\begin{tabular}{|c|c|c|c|c|c|}
\hline \multirow[b]{2}{*}{$n=149$} & \multicolumn{4}{|c|}{ Treatment group } & \multirow[b]{2}{*}{$p^{2}$} \\
\hline & $\begin{array}{c}\text { Enriched Diet } \\
\quad(n=35)\end{array}$ & $\begin{array}{c}\% \text { 5-MTHF } \\
(n=39)\end{array}$ & $\begin{array}{c}\text { Folic acid } \\
(n=37)\end{array}$ & $\begin{array}{l}\text { Placebo } \\
(n=38)\end{array}$ & \\
\hline Age (years) & $41(33,51)$ & $41(25,50)$ & $40(28,50)$ & $41(31,51)$ & 0.87 \\
\hline Sex, male $(\%)$ & 54.3 & 59.0 & 62.2 & 57.9 & 0.93 \\
\hline BMI $\left(\mathrm{kg} / \mathrm{m}^{2}\right)$ & $23.8(21.6,26.5)$ & $23.4(21.5,25.9)$ & $24.3(21.0,26.8)$ & $23.7(22.1,25.8)$ & 0.83 \\
\hline Weight (kg) & $70.5(60.5,84.5)$ & $66.0(55.5,79)$ & $71.5(58,83)$ & $67.0(56.5,79.0)$ & 0.64 \\
\hline Height $(\mathrm{cm})$ & $171(161,177)$ & $168(162,179)$ & $172(165,176)$ & $168(160,179)$ & 0.95 \\
\hline Current smokers $(\%)$ & 28.6 & 33.3 & 29.7 & 36.8 & 0.87 \\
\hline High educational level (\%) & 74.3 & 76.9 & 83.8 & 63.2 & 0.22 \\
\hline Health care professionals (\%) & 28.6 & 38.5 & 40.5 & 26.3 & 0.34 \\
\hline Physical active (\%) & 37.1 & 43.6 & 32.4 & 52.6 & 0.29 \\
\hline Vegetables eaters $(\%)$ & 51.4 & 41.0 & 43.2 & 52.6 & 0.81 \\
\hline Wine drinkers $(\%)$ & 51.4 & 69.2 & 56.8 & 65.8 & 0.28 \\
\hline Coffee drinkers $(\%)$ & 22.9 & 30.8 & 40.5 & 26.3 & 0.23 \\
\hline MTHFR polymorphism (\%) & & & & & 0.98 \\
\hline Homozygotes for wild-type & 42.9 & 33.3 & 32.4 & 34.2 & \\
\hline Heterozygotes & 31.4 & 38.5 & 40.5 & 39.5 & \\
\hline Homozygotes for variant allele & 25.7 & 28.2 & 27.1 & 26.3 & \\
\hline
\end{tabular}

${ }^{1}$ Continuous skewed are presented as median (1st, 3rd quartile) and categorical as frequencies; ${ }^{2} p$-values for differences between treatment groups derived through Pearson's $X^{2}$ for categorical variables and Kruskal-Wallis test for continuous skewed data.

Table 2. Baseline blood biomarkers of the participants according to treatment group ${ }^{1}$.

\begin{tabular}{lccccc}
\hline \multirow{2}{*}{$\boldsymbol{n}=\mathbf{1 4 9}$} & \multicolumn{5}{c}{ Treatment group } \\
\cline { 2 - 6 } & $\begin{array}{c}\text { Enriched diet } \\
(\boldsymbol{n}=\mathbf{3 5})\end{array}$ & $\begin{array}{c}\mathbf{\%} \text { 5-MTHF } \\
(\boldsymbol{n}=\mathbf{3 9})\end{array}$ & $\begin{array}{c}\text { Folic acid } \\
(\boldsymbol{n}=\mathbf{3 7})\end{array}$ & $\begin{array}{c}\text { Placebo } \\
(\boldsymbol{n}=\mathbf{3 8})\end{array}$ & $\boldsymbol{p}^{\mathbf{2}}$ \\
\hline Homocystein $(\mu \mathrm{mol} / \mathrm{L})$ & $14.3(11,17.3)$ & $14(11.1,18.1)$ & $13.3(11.6,15.3)$ & $14.8(10.1,17.2)$ & 0.96 \\
Red blood cells $(\mathrm{millions})$ & $4.64(4.32,5.15)$ & $4.89(4.43,5.34)$ & $4.91(4.60,5.19)$ & $4.76(4.43,4.97)$ & 0.32 \\
Hemoglobin $(\mathrm{g} / \mathrm{dL})$ & $13.5(12.9,14.7)$ & $13.8(13.0,15.2)$ & $14.7(13.2,15.1)$ & $14.4(13.6,14.7)$ & 0.42 \\
Haematocrit $(\%)$ & $42.2(39.3,45.9)$ & $42.4(40.0,47.2)$ & $44.6(40.5,46.3)$ & $43.5(40.5,44.9)$ & 0.65 \\
Creatinine $(\mathrm{mg} / \mathrm{dL})$ & $0.8(0.7,0.9)$ & $0.8(0.7,1.0)$ & $0.8(0.7,1.0)$ & $0.8(0.7,0.9)$ & 0.53 \\
Serum B6 $(\mathrm{ng} / \mathrm{mL})$ & $9.0(6.4,12.8)$ & $9.3(6.2,10.9)$ & $8.0(5.1,12.5)$ & $8.7(6.7,11.9)$ & 0.55 \\
Serum B12 $(\mathrm{pg} / \mathrm{mL})$ & $388(329,494) *$ & $310(252,437)$ & $307(250,388)$ & $311(225,386)$ & 0.04 \\
\hline
\end{tabular}

${ }^{1}$ Continuous skewed are presented as median (1st, 3rd quartile); ${ }^{2} p$-values for differences between treatment groups derived through and Kruskal-Wallis test for continuous skewed data; * Significantly different from the other three groups.

Levels of folate intake at baseline and after supplementation are shown in Supplementary Table S1. The mean intake of food folate was $212.2 \pm 55.1 \mu \mathrm{g}$ /day and did not differ, at baseline, across groups. After the supplementation period, food folate intake increased by $71 \%$ after 13 weeks of a diet 
enriched in folate. The increase was mainly due to an increased intake of fruit and vegetables, while the intake of other food groups did not change compared to the other treatment groups (Figure 1).

Figure 1. Folate intake through main food groups after supplementation, by treatment group.

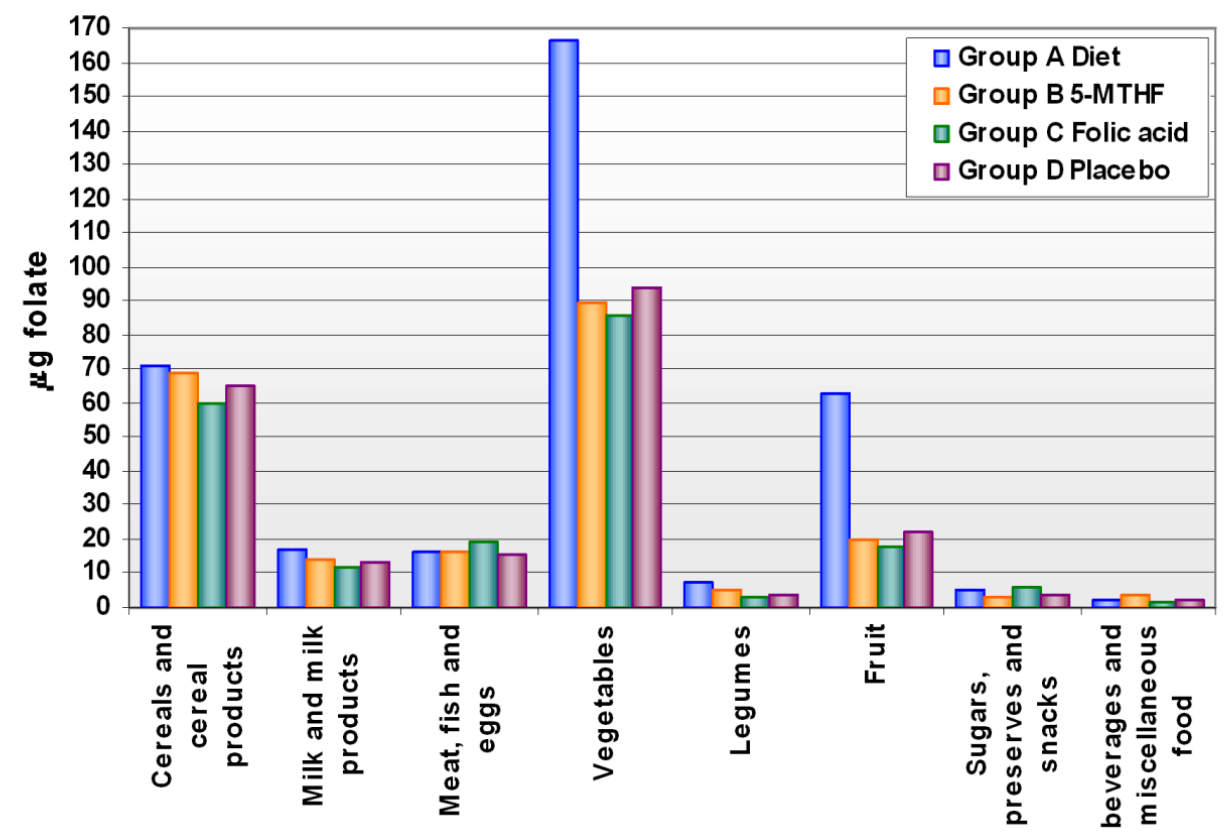

Table 3. Distribution of Hcy levels before and after the intervention according to treatment groups.

\begin{tabular}{|c|c|c|c|c|c|c|}
\hline Treatment group & $\begin{array}{c}\text { Pre-treatment } \\
\text { Homocysteine }^{1} \\
(\mu \mathrm{mol} / \mathrm{L})\end{array}$ & $\begin{array}{c}\text { Post-treatment } \\
\text { Homocysteine }^{1} \\
(\mu \mathrm{mol} / \mathrm{L})\end{array}$ & $\begin{array}{c}\% \text { Change after } \\
\text { treatment }^{2}\end{array}$ & $p^{3}$ & $p^{4}$ & $\begin{array}{c}\text { Homocysteine } \\
\text { decrease } \\
>25 \%(\%) \\
\end{array}$ \\
\hline Enriched Diet $(n=35)$ & $13.9(12.2,15.9)$ & $11.1(9.5,12.9)$ & -20.1 & 0.002 & $<0.001$ & 42.9 \\
\hline 5-MTHF $(n=39)$ & $13.9(12.2,15.9)$ & $11.2(9.8,12.8)$ & -19.4 & 0.001 & 0.002 & 46.2 \\
\hline Folic acid $(n=37)$ & $13.7(12.5,15.1)$ & $10.7(9.6,11.9)$ & -21.9 & $<0.001$ & $<0.001$ & 48.7 \\
\hline Placebo $(n=38)$ & $14.6(12.7,16.8)$ & $15.3(13.2,17.7)$ & 4.8 & 0.23 & & 10.5 \\
\hline
\end{tabular}

${ }^{1}$ Pre- and post-treatment homocysteine are presented as geometric means $(95 \% \mathrm{CI}) ;{ }^{2} \%$ change after treatment: The post treatment geometric mean divided by the pretreatment geometric mean; ${ }^{3} p$-values derived for differences in pre- and post-treatment homocysteine for each treatment group, through Wilcoxon's test; ${ }^{4} p$-values derived for differences in median homocysteine change after treatment between groups of treatment and placebo scheme through, Mann-Whitney's test.

In Table 3, changes in Hcy concentrations after the various interventions are shown. Hcy concentrations were reduced after folate enriched diet, 5-MTHF or folic acid supplementation by 20.1 ( $p<0.002), 19.4(p<0.001)$ and 21.9\% $(p<0.001)$ respectively, as compared to baseline levels, whereas an increase in Hcy concentrations by $4.8 \%$ (n.s.) was observed after placebo. Changes induced by interventions were significantly different from changes after placebo $(p<0.001, p<0.002$ 
and $p<0.001$, respectively for enriched diet, 5-MTHF and folic acid). There were no significant differences across treatments.

We also considered the number of subjects who reached a decrease in basal Hcy levels of at least $25 \%$. This target was reached by $42.9 \%, 46.2 \%, 48.7 \%$ and $10.5 \%$ of subjects treated respectively with enriched diet, 5-MTHF, folic acid and placebo. Multiple logistic regression analysis adjusted for sex, BMI, MTHFR genotypes, RBC folate, vitamin B6 and vitamin B12, shows an increased odd to reach a good response (Hcy levels <25\%) after enriched diet, 5-MTHF or folic acid of 7.6 (3.9-42.6), 7.9 (4.2-43.0) or 8.2 (5.9-48.5), respectively, as compared to placebo.

As expected, subjects homozygotes for the MTHFR TT genotype tended to have higher, although not significant, levels of Hcy than carriers the $\mathrm{C}$ allele (CC and CT) among three of the treatment groups, while a reverse pattern was seen for the5-MTHF treatment group. After enriched diet and folic acid supplementation, a decrease in Hcy concentrations was observed in both genotypes although it was higher in TT homozygotes (Table 4).

Table 4. Distribution of Hcy levels before and after the intervention according to treatment groups and MTHFR genotypes.

\begin{tabular}{|c|c|c|c|c|c|c|}
\hline $\begin{array}{l}\text { Treatment } \\
\text { group }\end{array}$ & $\begin{array}{c}\text { MTHFR } \\
\text { polymorphism }\end{array}$ & $\begin{array}{c}\text { Pre-treatment } \\
\text { Homocysteine } \\
(\mu \mathrm{mol} / \mathrm{L})\end{array}$ & $\begin{array}{c}\text { Post-treatment } \\
\text { Homocysteine }^{1} \\
(\mu \mathrm{mol} / \mathrm{L})\end{array}$ & $\begin{array}{c}\% \text { Change after } \\
\text { treatment }^{2}\end{array}$ & $p^{3}$ & $p^{4}$ \\
\hline \multirow{2}{*}{ Enriched Diet } & $T T(\mathrm{n}=9)$ & $15.1(10.6,21.4)$ & $11.3(7.8,16.3)$ & -25.2 & \multirow{2}{*}{0.46} & 0.07 \\
\hline & $C T / C C(\mathrm{n}=26)$ & $13.5(11.7,15.7)$ & $11.0(9.2,13.2)$ & -18.5 & & 0.01 \\
\hline \multirow{2}{*}{ 5-MTHF } & $T T(\mathrm{n}=11)$ & $12.8(9.1,18.2)$ & $11.8(8.9,15.6)$ & -7.8 & \multirow{2}{*}{0.47} & 0.37 \\
\hline & $C T / C C(\mathrm{n}=28)$ & $14.3(12.4,16.5)$ & $11.0(9.4,12.8)$ & -23.1 & & 0.002 \\
\hline \multirow{2}{*}{ Folic acid } & $T T(\mathrm{n}=10)$ & $15.3(11.6,20.3)$ & $10.6(8.1,14.0)$ & -30.7 & \multirow{2}{*}{0.35} & 0.01 \\
\hline & $C T / C C(\mathrm{n}=27)$ & $13.2(12.0,14.5)$ & $10.7(9.4,12.1)$ & -18.9 & & 0.001 \\
\hline \multirow{2}{*}{ Placebo } & $T T(\mathrm{n}=10)$ & $17.4(13.1,23.2)$ & $21.0(16.1,27.4)$ & 20.7 & \multirow{2}{*}{0.07} & 0.15 \\
\hline & $C T / C C(\mathrm{n}=28)$ & $13.7(11.7,16.1)$ & $13.7(11.6,16.1)$ & 0.21 & & 0.62 \\
\hline
\end{tabular}

\footnotetext{
${ }^{1}$ Pre- and post-treatment homocysteine are presented as geometric means $(95 \% \mathrm{CI}) ;{ }^{2} \%$ change after treatment: The post treatment geometric mean divided by the pretreatment geometric mean; ${ }^{3} p$-values for differences in Pre-treatment homocysteine levels among MTHFR-polymorphism groups derived through Mann-Whitney test.; ${ }^{4} p$-values derived for differences in pre- and post-treatment homocysteine for each treatment group and each MTHFR polymorphism, through Wilcoxon's test.
}

After 13 weeks of folate supplementation, a statistically significant increase in mean RBC folate concentration was observed only after folic acid supplementation; while in the other two treatment groups the increase was small and not significant (Table 5). The geometric mean concentration of RBC folate increased by $9.7 \%, 3.8 \%$ and $16.1 \%$ after enriched diet, 5-MTHF and folic acid, respectively. Both enriched diet and folic acid intake determined a significant increase in RBC folate concentration as compared to placebo ( $p<0.02$ and 0.01 , respectively) while the increase induced by 5 -MTHF intake was not significant. 
Table 5. Distribution of serum RBC levels before and after the intervention according to treatment group.

\begin{tabular}{|c|c|c|c|c|c|}
\hline Treatment group & $\begin{array}{l}\text { Pre-treatment } \\
\text { RBC folate }{ }^{1}\end{array}$ & $\begin{array}{l}\text { Post-treatment } \\
\text { RBC folate }{ }^{1}\end{array}$ & $\begin{array}{c}\% \text { Change after } \\
\text { treatment }^{2}\end{array}$ & $p^{3}$ & $p^{4}$ \\
\hline Enriched Diet $(n=33)$ & $225(210,241)$ & $247(223,275)$ & 9.7 & 0.12 & 0.02 \\
\hline 5-MTHF $(n=38)$ & $218(205,233)$ & $226(205,250)$ & 3.8 & 0.53 & 0.24 \\
\hline Folic Acid $(n=37)$ & $218(199,239)$ & $253(228,280)$ & 16.1 & 0.01 & 0.01 \\
\hline Placebo $(n=37)$ & $227(211,243)$ & $216(191,243)$ & -4.8 & 0.35 & \\
\hline
\end{tabular}

${ }^{1}$ Pre- and post-treatment RBC folate are presented as arithmetic means $(95 \% \mathrm{CI}) ;{ }^{2} \%$ change after treatment: The post treatment geometric mean divided by the pretreatment geometric mean; ${ }^{3} p$-values derived for differences in pre- and post-treatment RBC folate for each treatment group, through Wilcoxon's test; ${ }^{4} p$-values derived for differences in \% change of RBC folate after treatment between groups of treatment and placebo scheme, through Mann-Whitney's test.

\section{Discussion}

Different folic acid supplementations by enriched diet $(200 \mu \mathrm{g} / \mathrm{day}), 5-\mathrm{MTHF}$ (200 $\mu \mathrm{g} / \mathrm{day}$, $340 \mathrm{DFE})$, or folic acid (200 $\mu \mathrm{g}$ /day, $340 \mathrm{DFE})$, induced a significant reduction in Hcy concentrations in comparison with the control group.

The efficacy of 5-MTHF has been compared with that of folic acid in several studies with contrasting results: Fohr et al. [16] showed that in women folic acid was more effective than MTHF in lowering plasma total Hcy, while Venn et al. [17] reported that a low-dose of 5-MTHF was at least as effective as folic acid in reducing total Hcy concentrations in healthy subjects. In contrast, 5-MTHF was more effective than folic acid in increasing plasma folate levels in the study of Prinz-Langenohl [18] both in TT and CC subjects.

The decrease in Hcy concentration was only partially influenced by the MTHFR genotype; indeed enriched diet and folic acid were more effective in decreasing Hcy levels in TT homozygotes as compared with carriers of $\mathrm{C}$ allele. Our data partially agree with those of Fohr et al. [16] who reported the most pronounced total Hcy-lowering effect occurring in women with the TT genotype. The smaller percent change in Hcy observed in the TT subjects treated with 5-MTHF, could be due to the low baseline value rather than the genotype. However, the small number of subjects with different genotypes in each treatment group should be considered.

We demonstrated that an adequate folate status could also be achieved by eating a greater amount of natural folate-rich foods, even if the bioefficacy of folate from some foods is significantly lower than that of folic acid itself; the bioefficacy of natural folate, in fact, is closely dependent on the food matrix and on other factors. However, bioefficacy of folate derived from vegetables and citrus fruit ranges from $60 \%$ to about $100 \%$ of that of folic acid $[8,19]$. In our study, the enrichment of diet with folate was mainly obtained by introducing more fruit and vegetables, while the consumption of other food groups rich in folate was similar to the other supplementation groups.

A limitation of our study is the use of RBC folate concentration to evaluate the folate status; indeed, although it is usually considered a better index of body folate stores, the short duration of the intervention period, makes $\mathrm{RBC}$ folate concentration only partially reflecting post-supplementation folate status. This could explain the small changes in RBC folate concentration observed after 
treatment. Our results show a stronger effect in increasing RBC folate concentration of folic acid $(+16.1 \%)$, followed by natural folate enriched diet $(+9.7 \%)$ whereas a lower percent variation was observed with the 5-MTHF treatment $(+3.8 \%)$. These data are apparently at variance with those of other studies, which used, however, different supplementation protocols: in the study of Fohr et al. [16] (FA and 5-MTHF $400 \mu \mathrm{g} /$ day, for 8 weeks), the post-treatment variation in RBC folate was $+63.4 \%$ and $+59.7 \%$, respectively. Cuskelly et al. [20] found a variation of $+40.2 \%$ and $+52 \%$ after supplementation with $400 \mu \mathrm{g} /$ day of folic acid and fortified foods respectively for 12 weeks; the same authors, in the group supplemented with $400 \mu \mathrm{g} /$ day of natural folate enriched diet, found a lower increase in the concentration of RBC folate $(+11 \%)$. In addition, in the study of Venn [17], after a lower supplementation (folic acid and 5-MTHF $100 \mu \mathrm{g} / \mathrm{day}$, for 24 weeks), the percentage of variation after 24 weeks of treatment was $+31 \%$ and $+23 \%$, respectively.

In our study, daily folate enrichment of $200 \mu \mathrm{g}$ /day of the usual diet (containing about $220 \mu \mathrm{g} /$ day) with the three strategies adopted (increased consumption of folate rich foods, pill of 5-MTHF, pill of folic acid) resulted effective in lowering significantly serum Hcy, however only folic acid supplementation was able to increase RBC folate in subjects with mild hyperhomocysteinemia. Mean typical folate intake in Italy is $220 \mu \mathrm{g} /$ day, with a suggested minimum intake of $200 \mu \mathrm{g} / \mathrm{day}$. Increasing folate intake to $400 \mu \mathrm{g}$ /day achieves near-maximal Hcy reduction and has been proposed as the minimum recommended folic acid intake for NTDs (neural tube defects) prevention [21]. In Italy, fortification with folic acid is not mandatory, although there is an increasing trend for food manufacturers to voluntarily fortify breakfast cereals, which are not frequently used by the general population [22]. We planned our study to increase the daily intake of folate to the optimal level of $400 \mu \mathrm{g}$ /day by adding to the usual dietary intake foods rich in folate, or two different pharmacological forms of folic acid. Our study demonstrated that, increasing the consumption of fruit and vegetable is effective in decreasing Hcy concentrations in a comparable extent to the pharmacological supplementations. Our data are in agreement with other studies showing that an increase in folic acid intake up to $200 \mu \mathrm{g}$ /day may result as effective in reducing tHcy concentration as supplementation at higher levels [23,24]. The majority of the studies that investigated about folate intake and Hcy concentration, used pharmacological supplementation (folic acid, 5-MTHF) or folic acid fortified foods; not many studies considered the effects of natural folate enriched diet [25-27]. Our data partially agree with those obtained by Ahfield-Watt et al. [25] which showed a similar Hcy lowering effect of folate enriched diet $(-14 \%)$ and folic acid supplements $(-16 \%)$; the post-treatment variation in folate status is not easily comparable because these authors measured levels of folate only in plasma. It is however interesting to note that a significant increase in folate levels and a significant reduction in Hcy concentrations were obtained also with the enriched diet, although the enriched diet in this study included not only natural folate but also folic acid fortified foods. Therefore, a folate rich-diet could be suggested to free-living population to maintain an adequate folate status, also taking into account that it provides, beside folates, other healthy nutrients such as antioxidants and other vitamins [20].

Although our results show that the supplementation with natural folate rich foods, in a free-living mediterranean population, reached a reduction in Hcy concentrations, comparable to that obtained with pharmacological supplements (folic acid and 5-MTHF), it's difficult to imagine that this could be the best and simplest strategy to reach and to maintain for a longer period, an adequate folate status; the 
pharmacological supplementation certainly assures more prompt effects especially in particular conditions such as pregnancy planning, in which a rapid improvement in folate status is desirable to prevent adverse pregnancy outcome and NTDs.

\section{Conclusions}

Our results show that the supplementation with natural folate rich foods, folic acid and 5-MTHF in a free-living mediterranean population, reached a similar reduction in Hcy concentrations. However, it could be advisable to prefer a pharmacological supplementation in particular conditions such as pregnancy.

\section{Acknowledgements}

This study was partially supported by the European Union grant QLRT-1999-00576 \& QLRT-2001-2740.

\section{Conflict of Interest}

The authors declare no conflict of interest.

\section{References}

1. Clarke, R.; Halsey, J.; Bennett, D.; Lewington, S. Homocysteine and vascular disease: Review of published results of the homocysteine-lowering trials. J. Inherit. Metab. Dis. 2011, 34, 83-91.

2. Dangour, A.D.; Withouse, P.J.; Rafferty, K.; Mitchell, S.A.; Smith, L.; Hawkesworth, S.; Vellas, B. B-vitamins and fatty acids in the prevention and treatment of Alzheimer's disease and dementia: A systematic review. J. Alzheimers Dis. 2010, 22, 205-224.

3. Wald, D.S.; Kasturiratne, A.; Simmonds, M. Effect of folic acid, with or without other B vitamins, on cognitive disorders: Meta-analysis of randomized trials. Am. J. Med. 2010, 123, 522-527.e2.

4. Kim, Y.I. Folate and colorectal cancer: An evidence based critical review. Mol. Nutr. Food Res. 2007, 51, 267-292.

5. Eussen, S.J.P.M.; Vollset, S.E.; Ingland, J.; Meyer, K.; Fredriksen, A.; Ueland, P.M.; Jenab, M.; Slimani, N.; Boffetta, P.; Overvad, K.; et al. Plasma folate, related genetic variants and colorectal cancer risk in EPIC. Cancer Epidemiol. Biomark. Prev. 2010, 19, 1328-1340.

6. De-Regie, L.M.; Fernandez-Gaxiola, A.C.; Donswell, T.; Pena-Rosas, J.P. Effects and safety of periconceptional folate supplementation for preventing birth defects. Cochrane Database Syst. Rev. 2010, CD007950, doi:10.1002/14651858.CD007950.pub2.

7. Molloy, A.M.; Kirke, P.N.; Brody, L.C.; Scott, J.M.; Mills, J.L. Effects of folate and vitamin B12 deficiencies during pregnancy on fetal, infant and child development. Food Nutr. Bull. 2008, 29 (2 Suppl.), S101-S111.

8. Brouwer, I.A.; van Dusseldorp, M.; West, C.E.; Steegers-Theunissen, R.P.M. Bioavailability and bioefficacy of folate and folic acid in man. Nutr. Res. Rev. 2001, 14, 267-293. 
9. Dhonukshe-Rutten, R.A.M.; de Vries, J.H.M.; de Bree, A.; van der Put, N.; van Staveren, W.A.; de Groot, L.C.P.G.M. Dietary intake and status of folate and vitamin B12 and their association with homocysteine and cardiovascular disease in European populations. Eur. J. Clin. Nutr. 2009, 63, 18-30.

10. Turrini, A.; Leclercq, C.; D'Amicis, A. Patterns of food and nutrient intakes in Italy and their application to the development of food-based dietary guidelines. Br. J. Nutr. 1999, 81 (Suppl. 2), S83-S89.

11. Institute of Medicine. DRI Dietary References Intake for Thiamin, Riboflavin, Niacin, Vitamin B6, Folate, Vitamin B12, Pantothenic Acid, Biotin and Choline; National Academy Press: Washington, DC, USA, 1998.

12. Araki, A.; Sako, Y. Determination of free and total homocysteine in human plasma by high-performance liquid chromatography with fluorescence detector. J. Chromatogr. 1987, 422, 43-52.

13. De Stefano, V.; Zappacosta, B.; Persichilli, S.; Rossi, E.; Casorelli, I.; Paciaroni, K.; Chiusolo, P.; Leone, A.M.; Giardina, B.; Leone, G.; et al. Prevalence of mild hyperhomocisteinemia and association with thrombophilic genotypes (Factor V Leiden and factor II G20210A) in Italian patients with venous thromboembolic disease. Br. J. Haematol. 1999, 106, 564-568.

14. Wright, A.J.A.; Finglas, P.M.; Southon, S. Erythrocyte folate analysis: Saponin added during lysis of whole blood can increase apparent folate concentrations, depending on hemolysate $\mathrm{pH}$. Clin. Chem. 2000, 46, 1978-1986.

15. Frosst, P.; Blom, H.J.; Milos, R.; Goyette, P.; Sheppard, C.A.; Matthews, R.G.; Boers, G.J.; den Heijer, M.; Kluijtmans, L.A.; van den Heuvel, L.P.; et al. A candidate genetic risk factor for vascular disease: A common mutation in methylenetetrahydrofolate reductase. Nat. Genet. 1995, 10, 111-113.

16. Fohr, I.P.; Prinz-Langenohl, R.; Brönstrup, A.; Bohlmann, A.M.; Nau, H.; Berthold, H.K.; Pietrzik, K. 5,10-Methylenetetrahydrofolate reductase genotype determines the plasma homocysteine-lowering effect of supplementation with 5-methyltetrahydrofolate or folic acid in healthy young women. Am. J. Clin. Nutr. 2002, 75, 275-282.

17. Venn, B.J.; Green, T.J.; Moser, R.; Mann, J.I. Comparison of the effect of low-dose supplementation with L-5-methyltetrahydrofolate or folic acid on plasma homocysteine: A randomized placebo-controlled study. Am. J. Clin. Nutr. 2003, 77, 658-662.

18. Prinz-Langenohl, R.; Brämswig, S.; Tobolski, O.; Smulders, Y.M.; Smith, D.E.C.; Finglas, P.M.; Pietrzik, K. [6S]-5-methyltetrahydrofolate increases plasma folate more effectively than folic acid in women with the homozygous or wild-type $677 \mathrm{C} \rightarrow \mathrm{T}$ polymorphism of methylenetetrahydrofolate reductase. Br. J. Pharmacol. 2009, 158, 2014-2021.

19. Brouwer, I.A.; van Dusseldorp, M.; West, C.E.; Meyboom, S.; Thomas, C.M.G.; Duran, M.; van het Hof, K.H.; Eskes, T.K.; Hautvast, J.G.; et al. Dietary folate from vegetables and citrus fruit decreases plasma homocysteine concentrations in humans in a dietary controlled study. J. Nutr. 1999, 129, 1135-1139.

20. Cuskelly, G.J.; McNulty, H.; Scott, J.M. Effect of increasing dietary folate on red-cell folate: Implications for prevention of neural tube defects. Lancet 1996, 347, 657-659. 
21. Homocysteine Lowering Trialists' Collaboration. Lowering blood homocysteine with folic acid-based supplements: Meta-analysis of randomised trials. BMJ 1998, 316, 894-898.

22. Zappacosta, B.; Persichilli, S.; Iacoviello, L.; di Castelnuovo, A.; Graziano, M.; Gervasoni, J.; Leoncini, E.; Cimino, G.; Mastroiacovo, P. Folate, vitamin B12 and homocysteine status in an Italian blood donor population. Nutr. Metab. Cardiovasc. Dis. 2011, doi:10.1016/j.numecd.2011.10.001.

23. Ward, M.; McNulty, H.; McPartlin, J.; Strain, J.J.; Weir, D.G.; Scott, J.M. Plasma homocysteine, a risk factor for cardiovascular disease, is lowered by physiological doses of folic acid. Quart. J. Med. 1997, 90, 519-524.

24. Venn, B.J.; Mann, J.L.; Williams, S.M.; Riddell, L.J.; Chisholm, A.; Harper, M.J.; Aitken, W.; Rossaak, J.L. Assessment of three levels of folic acid on serum folate and plasma homocysteine: A randomised placebo-controlled double-blind dietary intervention trial. Eur. J. Clin. Nutr. 2002, 56, 748-754.

25. Ashfield-Watt, P.A.L.; Pullin, C.H.; Whiting, J.M.; Clark, Z.E.; Moat, S.J.; Newcombe, R.G.; Burr, M.L.; Lewis, M.J.; Powers, H.J.; McDowell, I.F.W. Methylenetetrahydrofolate reductase $677 \mathrm{C} \rightarrow \mathrm{T}$ genotype modulates homocysteine responses to a folate-rich diet or a low dose folic acid supplement: A randomized controlled trial. Am. J. Clin. Nutr. 2002, 76, 180-186.

26. Ashfield-Watt, P.A.L.; Whiting, J.M.; Clark, Z.E.; Moat, S.J.; Newcombe, R.G.; Burr, M.L.; McDowell, I.F.W. A comparison of the effect of advice to eat either "5-a-day" fruit and vegetables or folic acid-fortified foods on plasma folate and homocysteine. Eur. J. Clin. Nutr. 2003, 57, 316-323.

27. Bogers, R.P.; Dagnelie, P.C.; Bast, A.; van Leeuwen, M.; van Klaveren, J.D.; van den Brandt, P.A. Effect of increased vegetable and fruit consumption on plasma folate and homocysteine concentrations. Nutrition 2007, 23, 97-102.

\section{Supplementary}

Table S1. Food folate intake at baseline and after supplementation, by treatment groups.

\begin{tabular}{lcccc}
\hline Folate intake $\boldsymbol{\mu g} /$ day & Diet & 5-MTHF & Folic acid & Placebo \\
& $\boldsymbol{n}=\mathbf{3 5}$ & $\boldsymbol{n}=\mathbf{3 9}$ & $\boldsymbol{n}=\mathbf{3 7}$ & $\boldsymbol{n}=\mathbf{3 8}$ \\
\hline Baseline & $205.2 \pm 54.9$ & $220.5 \pm 44.9$ & $202.5 \pm 66.8$ & $220.5 \pm 53.9$ \\
After supplemetation & $350.9 \pm 76.1$ & $237.2 \pm 75.5$ & $236.2 \pm 75.7$ & $218.5 \pm 88.3$ \\
\hline
\end{tabular}

(C) 2013 by the authors; licensee MDPI, Basel, Switzerland. This article is an open access article distributed under the terms and conditions of the Creative Commons Attribution license (http://creativecommons.org/licenses/by/3.0/). 
Dietary patterns and fatty acids levels of three European populations. Results from the IMMIDIET study.

Pounis G, de Lorgeril M, Salen P, Laporte F, Krogh V, Siani A, Arnout J, Cappuccio FP, van Dongen M, Donati MB, de Gaetano G, lacoviello L, on behalf of the European Collaborative Group of the IMMIDIET Project. Dietary patterns and fatty acids levels of three European populations. Results from the IMMIDIET study. Nutr Met Card Dis, In Press. 


\section{Dietary patterns and fatty acids levels of three European populations. Results from the IMMIDIET study.}

George Pounis ${ }^{1}$, Michel de Lorgeril ${ }^{2}$, Patricia Salen ${ }^{2}$,François Laporte ${ }^{3}$,Vittorio Krogh $^{4}$, Alfonso Siani ${ }^{5}$,Jozef Arnout ${ }^{6}$,Francesco P Cappuccio ${ }^{7}$, Martien van Dongen ${ }^{8}$, Maria Benedetta Donati ${ }^{1}$, Giovanni de Gaetano ${ }^{1}$, Licia lacoviello ${ }^{1}$, on behalf of the European Collaborative Group of the IMMIDIET Project

${ }^{1}$ Department of Epidemiology and Prevention, IRCCS Istituto Neurologico Mediterraneo Neuromed, Pozzilli (IS), Italy

${ }^{2}$ Cœur et Nutrition, PRETA, TIMC-IMAG CNRS 5525, Université Joseph Fourier-CNRS, Faculté de Médecine, La Tronche, France

${ }^{3}$ Département de Biochimie, Pharmacologie et Toxicologie, Hôpital Universitaire, Grenoble, France,

${ }^{4}$ Istituto Nazionale dei Tumori, Milan, Italy

${ }^{5}$ Institute of Food Sciences, CNR, Avellino, Italy

${ }^{6}$ Katholieke Universiteit Leuven, Belgium

${ }^{7}$ Warwick Medical School, Coventry, United Kingdom

${ }^{8}$ Department of Epidemiology, Maastricht University, Maastricht, The Netherlands

IMMIDIET Project Investigators are listed in the supplementary material

Corresponding Author and address for reprints requests: Licia lacoviello, MD, PhD, Laboratory of Molecular and Nutritional Epidemiology, Department of Epidemiology and Prevention, IRCCS Istituto Neurologico Mediterraneo Neuromed, Via Dell'Elettronica, 86077 Pozzilli (IS), Italy; E-mail: licia.iacoviello@moli-sani.org; Tel:+390865929664; Fax:+390865927575.

List of abbreviations: RRR,reduced rank regression;FA, fatty acid; CVD, cardiovascular disease; RBC, red blood cell; EPIC, European Prospective Investigation into Cancer and Nutrition; FFQ, food frequency questionnaire; NAF, Nutrition Analysis of FFQ; FCT, food composition tables; BP, blood pressure; SBP, systolic blood pressure; DBP, diastolic blood pressure; BMI, body mass index; LDL, low-density lipoprotein.

Word count (Title page to acknowledgement): 2944 


\begin{abstract}
Background and Aims: Differences in blood FAs profile among populations with different lifestyle have partly been attributed to differences in food intake. A holistic approach in dietary guidance through dietary patterns is essential. This study aimed at evaluating the main plasma and RBC FAs in three European populations and assessing the role of dietary patterns in explaining variation in their levels. Methods: In the framework of the IMMIDIET Project, 1,604 subjects (802 male female pairs) aged 26-65 years were enrolled in Italy, Belgium and UK. Plasma and RBC FAs were measured. 1-year recall FFQs were used to evaluate dietary habits of each individual. Results: Italians showed lower plasma and RBC n3 levels than participants of the other two populations ( $P$ for all<0.001). Both plasma and RBC arachidonic acid were higher in Italians as compared to Belgians and English. RRR analysis indicated two dietary patterns explaining $35 \%$ and $17 \%$ of the total variation of the sum of plasma and RBC n-3, respectively. In a holistic dietary analysis, neither fish nor mollusks intake seemed to contribute to $\mathrm{n}-3$ variation as compared to vegetable oils and polyphenolrich foods. Conclusion: Italian participants had lower plasma and RBC n-3 levels compared to other countries. This could in part be attributed to differences in dietary pattern adherence. A holistic approach dietary analysis that takes into account FAs levels may contribute to public health promotion strategies.
\end{abstract}




\section{INTRODUCTION}

The majority of studies evaluating possible associations of dietary habits with health outcomes focus on the effects of single nutrients or foods on health or illness. However, the complexity of the human diet, especially the interactions between foods or nutrients, makes it more difficult to evaluate their effects on diseases or on biological predictors or risk factors. Recently, new statistical methods such as RRR have been proposed to study the combined effect of foods on health outcomes. As an "a posteriori" method, it includes the construction of dietary patterns derived from the data at hand; thus determining combinations of food intake that explain as much response variation as possible in specific populations ${ }^{1}$.

Growing evidence suggests that blood FAs are major biomarkers-predictors and even causal factors for many diseases, in particular cardiovascular disease (CVD), cancer and nervous system disease ${ }^{2-5}$. In that context, omega-3 FAs (n-3) and their interactions with omega-6 FAs (n-6) are obviously critical ${ }^{2,3}$.

Consumption of n-3, EPA (20:5n-3) and DHA (22:6n-3), may improve cardiovascular health by altering FA metabolism, inducing hemodynamic changes, decreasing arrhythmias, modulating platelet function, improving endothelial function and inhibiting inflammatory pathways ${ }^{2,3}$. N-3 deficiency or insufficiency and low blood/tissue n-3 levels were associated with increased disease risks ${ }^{2,3,6}$

Most population differences in prevalence and severity of CVD such as between Northern European vs. Mediterranean populations, could in part be explained by population differences in lifestyle and dietary habits ${ }^{7}$. Types of fats consumed are among the possible factors explaining the relations between dietary habits and CVD risk ${ }^{8}$.

Despite the relation of FAs levels with the consumption of some food groups, there is limited knowledge on the possible association of whole dietary patterns with blood/tissue FA profiles. Moreover, there is apparently no comparative data on the levels of main FAs in blood/tissue across different populations such as Northern vs. Southern Europeans neither on dietary patterns that may explain differences in FA profiles.

This study aimed at evaluating the main plasma and RBC FAs levels in three European populations at different dietary habits and risk of CVD. We also evaluated the role of dietary patterns in relation to the observed variation in $n-3$ blood/tissue [plasma/red blood cells $(\mathrm{RBC})]$ levels.

\section{SUBJECTS AND METHODS Participants}

The IMMIDIET Project and the recruitment of subjects were previously described ${ }^{7}$. Between October 2001 and October 2003,271 male-female pairs in the Abruzzo region in Italy, 268 pairs in the Flemish territory of Belgium and 263 in S-W London in England, aged 26-65 years were enrolled in this cross-sectional study. Eligible pairs were randomly selected in each country, by local general practitioners. The participation rate ranged between $70-90 \%$ in the different centres. The ethical committees of all participating institutions approved the study. All study participants agreed by written informed consent.

\section{Dietary and lifestyle assessment}

Interviews were taken using a standardised questionnaire previously adopted in the Olivetti Prospective Heart Study ${ }^{9}$.

The validated Italian and English EPIC-FFQ ${ }^{10}$ were used to evaluate dietary intake of 164 food items over the past year. Since a valid EPIC-FFQ version was not available for the Belgian subpopulation a new 322-item FFQ was developed to assess the food intake of the Belgian-Belgian couples ${ }^{11}$. A computer program, $\mathrm{NAF}^{12}$ converted questionnaire dietary data into frequencies of consumption and average daily quantities of foods, energy and nutrients consumed. NAF was linked to the McCanceFCT ${ }^{13}$, the Italian FCT for Epidemiological studies $^{14}$, and the Dutch NEVO ${ }^{15}$ and Flemish-Belgian Nubel FCTs ${ }^{16}$.For the purpose of this 
study, the 164 food items formed the 20 basic food groups that were included in the dietary analysis.

\section{Clinical assessment and biochemical measurement of FAs}

Trained research personnel in the different recruitment centres performed blood pressure (BP) and anthropometric measurements. Systolic and diastolic BP were measured with an automated device (OMRON-HEM- 705CP; OMRON Corporation, Amsterdam, Netherlands) ${ }^{17}$. Body weight and height were measured in subjects without shoes and wearing light clothing with the use of a standard beam balance scale and attached ruler. $\operatorname{BMI}\left(\mathrm{kg} / \mathrm{m}^{2}\right)$ was calculated.

Blood samples were obtained between 7.00 and 9.00 a.m. from patients who had been fasting overnight and had refrained from smoking for at least 6 hours. Measurements of serum lipids and blood glucose levels were performed by an automated analyser (CobasMira-Plus, Roche, Milano Italy). LDL cholesterol was estimated by the Friedwald formula ${ }^{18}$.

Regarding FA measurements, blood samples (collected into EDTA), were separated into plasma and packed cells by centrifugation and kept frozen at $-70^{\circ} \mathrm{C}$ until FAs could be extracted. The FA composition in red blood cells was shown to remain stable for a long time at this temperature ${ }^{19}$.

Plasma and RBCFAs were measured by gas chromatography ${ }^{8}$. Lipids were extracted by a monophasic method that uses a solution of hexane to isopropanol $(3: 2 ; \mathrm{vol}: \mathrm{vol})$ after adding heptadecanoic acid as an internal standard. Extracted lipids were saponified and methylated with $14 \%$ boron trifluoride in alcohol. After extraction, the methylated FAs were quantified by gas chromatography with flame ionization detection on a capillary column. Hydrogen was the carrier gas. FA peaks were identified and quantified by comparison with known standards, and FA composition is reported as weight percentage of total FAs.RBC EPA and DHA reported as weight percentage of total FAs gave the "Omega 3 Index"

\section{Statistical analysis}

The normality of continuous variables was tested graphically and presented as mean (standard deviation). Plasma or RBCFA levels and dietary data were skewed so they are presented as median $\left(1^{\text {st }}-3^{\text {rd }}\right.$ quartiles). Comparisons of plasma or RBCFA levels between countries were performed using the one-way ANOVA F-test on log-transformed levels. After Bonferroni correction for multiple comparisons alpha $=0.003$ (17-tests) was established as significance cut-off point for both plasma and RBCFA differences between countries. Comparisons of clinical and anthropometric measurements between countries were performed using one-way ANOVA F-test; while comparisons of dietary data were done using Kruskal-Wallis test.

RRR was used in order to derive dietary patterns that better describe high blood $n-3$ levels. RRR works with two different sets of variables, called predictors and responses; it extracts linear functions of predictors (named factors) that explain as much response variation as possible ${ }^{1}$. In this study RRR-analysis was performed two times. In each of both analyses the predictors were 21 dietary components and the responses were the log-transformed total plasma $n-3$ FAs (ALA, EPA, DPA, DHA) valuesfor the $1^{\text {st }}$ model, and the log-transformed total RBCn-3 FAs (ALA, EPA, DPA, DHA) values for the $2^{\text {nd }}$ model. RRR-analysis produced each time one factor model-dietary pattern. The correlations between each extracted factor and dietary components are called factor loadings; as a rule of thumb, we characterized the factor using the dietary components with an absolute factor loading $\geq 0.20$. Each subject received, for each pattern, a factor score (RRR-score), calculated by summing the observed intakes of the 21 dietary components, each weighted by its factor loading ${ }^{1}$. A higher value of this score represents increased adherence to the factor-dietary pattern that describes better the high total plasma or RBC n-3 level. 
Spearman's rho was used to quantify the association between each dietary component indicated by the two extracted dietary patterns with total plasma or RBC n-3 levels. All tested hypotheses were two-sided. A P-value $<0.05$ was considered to reflect statistical significance. STATA version 9, software was used for all calculations except from RRR where SAS software version 9.1was used. PASW statistics software version 18.0 was used to produce histograms and normal curves.

\section{RESULTS}

\section{Plasma FAs levels}

Table 1 shows the distribution of individual plasma FAs according to country. A significant difference between the three countries was observed for the majority of them. Italians showed higher plasma levels of myristicand palmitic FAs but lower levels of arachidic acid as compared to Belgians ( $P$ for all pair differences $<0.001$ ). No significant difference was observed between Italians and English for any saturated FAs ( $P$ for all $>0.003$ ).

Plasma n-3ALA, EPA, and DPA levels were lower in Italians compared to either Belgian or English participants ( $P$ for all pair differences $<0.001<a^{\prime}=0.003$, Bonferroni correction for multiple comparisons) (Table 1).Plasma DHA was lower in Italians compared to Belgians or English; while levels in UK were the highest $(P$ for all pair differences $<0.001<a^{\prime}=0.003$ ). The trend of Italians to have lower plasma $n-3$ than the participants of the other two populations is also illustrated in Supplementary Figure S1.

The distribution of plasma n-6, n-7 and n-9 presented significant differences among the three countries with no specific trends (Table 1). N-6 LA was lower and AA and oleic acid were higher in Italy compared to Belgium or UK $\left(P\right.$ for all pair differences $\left.<0.001<a^{\prime}=0.003\right)$.

\section{RBC FAs levels}

The distribution of RBC FA levels according to country is illustrated in Table 2. Most of saturated and unsaturated RBC FAs followed the same trend observed for plasma FAs. RBC myristic acid levels were lower in Italians compared to Belgians and English. On the contrary, palmitic acid levels were in Italy higher than in Belgium and lower than in UK ( $P$ for all pair differences $<0.001<a^{\prime}=0.003$, Bonferroni correction for multiple comparisons). No significant differences were observed for stearic and arachidic acids ( $P$ for all $>0.003=a ')$.

RBC n-3ALA, EPA, and DPA were lower in Italians compared to Belgians and English ( $P$ for all pair differences $<0.001<a^{\prime}=0.003$, Bonferroni correction for multiple comparisons) (Table 2), while DHA levels differed only between English and Belgians ( $P$ for all pair differences $<0.001<a^{\prime}=0.003$ ) and no significant difference was observed with Italians $(P$ for pair differences $>a^{\prime}=0.003$ ). This was also evident for the omega- 3 index, which was significantly lower in Italians compared to Belgians or English $(P$ for all pair differences $<0.001<a^{\prime}=0.003$ ). The trend of Italians to have lower n-3 RBC compared with the two other populations is also illustrated in Supplementary Figure S2.

The distribution of n-6, n-7 and n-9 FA in RBC presented also significant differences among the three populations, although without specific trends (Table 2). N-6 LA (the substrate for the endogenous synthesis of the $n-6 A A)$ levels were lower in Italy compared to Belgium and UK whereas AA levels were higher in Italy ( $P$ for all pair differences $<0.001<a^{\prime}=0.002$ ).

\section{Dietary patterns in relation to $\mathrm{n}-3$ FAs levels}

RRR-analysis was used in order to derive dietary patterns that could fully describe the high plasma and RBC n-3 levels (Table 3).

Model 1 identified a factor-dietary pattern explaining $35 \%$ of the total variation of total plasma n-3. This pattern was described by high consumption of cabbages and root 
vegetables, dairies, vegetable oils, chocolate, coffee and tea and mayonnaise; and low intake of cheese, pasta and rice, red meat and processed products and olive oil.

The model 2 revealed a factor-dietary pattern explaining $17 \%$ of the total variation of total RBC n-3. This dietary pattern was described by high intake of cabbages and root vegetables, fishes, mollusks, vegetable oils, chocolate, coffee and tea and mayonnaise and low intake of pasta and rice, red meat and processed products and olive oil.

Cabbages and root vegetables, diaries, chocolate, coffee and tea, mayonnaise, fishes, mollusks and vegetable oils were individually positively associated with both plasma and RBC n-3 FA (Table 4, for all Spearman's rho $>0, P<0.05$ ), while pasta and rice, red meat and olive oil were negatively associated (for all Spearman's rho $<0, P<0.001$ ). The negative association with cheese was only significant for plasma n-3 FA but not for RBC n-3 FA (Spearman's rho $=-0.22, P<0.001$ and Spearman's rho $=-0.04, P=0.07$, respectively).

\section{Population differences in dietary patterns related to n-3 FAs}

Italians had lower RRR-scores in both models compared with Belgians and English (Table 5) $(P<0.001)$. The analysis of food group consumption confirmed the low adherence to the two dietary patterns identified by RRR-analysis. In particular, the intake of food groups that should be consumed mostly according to these patterns were significantly lower in Italy compared to Belgium or UK $(\mathrm{P}<0.001)$.

\section{DISCUSSION}

The main finding of this study comparing three European study populations is that there are striking differences inplasma and RBC FA profiles and, in particular, that Italian participants have lower plasma and RBC n-3 FA levels compared to Belgians and English. Apparently for the first time, dietary pattern analysis identified two dietary patterns associated with high plasma and RBC levels of n-3 FA, respectively. These patterns, explained the $35 \%$ and the $17 \%$ of the variation of plasma and RBC n-3 FA, correspondingly. Italians showed lower adherence compared to Belgians and English to both patterns, as confirmed by food group consumption analysis.

Plasma and RBC n-3 FA levels in Italians appeared to be low in absolute values too and close to those observed in patients with $C V D^{8}$. These levels were similar to those observed in Italian patients enrolled in the GISSI-HF trial ${ }^{20}$ and lower than those measured in the English population of the EPIC-Norfolk study ${ }^{21}$. The omega-3 index of these apparently healthy Italians would classify them as "at intermediate risk" for CVD".

The diet of Italians included in our study was by one side characterized by relatively high intakes of olive oil, fruits and leafy vegetables, tomatoes, pasta, rice and fish as compared with English and Belgians; on the other side, they were lower consumers of cabbages and root vegetables, diaries, vegetable oils, chocolate, coffee and tea and mayonnaise. In both n-3-inducing dietary patterns identified in this study, the consumption of these foods was associated with higher n-3 levels despite their low content in n-3 (except nuts), suggesting important interactions among substances present in these foods that may regulate the metabolism and the levels of $n-3$.

Some of the relations between foods and n-3 FAs observed in this study could be in part explained by differences in oil consumption. The three populations actually consumed different vegetable oils. Italians almost exclusively used olive oil, which is poor in both n-3 and $\mathrm{n}-6$ but is rich in oleic acid. In contrast, in UK and Belgium, participants consumed greater quantities of vegetable oils, margarines and mayonnaises that are all relatively high in $n-6$ and $n-3$. As ALA and LA are the precursors of the other long-chain $n-3$ and $n-6$ through endogenous elongation/desaturation ${ }^{2}$, this implies that all the other $n-3$ and $n-6$ FA would be different across the three populations.

Other observed associations between food intake and FAs blood levels are to be mentioned. Fish and mollusks are reportedly the main sources of EPA, DPA and DHA ${ }^{23}$. 
Italians consume more fish, twice the amount eaten by English, yet they show levels of plasma EPA, DPA and DHA about 50\% lower. Thus the most reasonable explanation for the low EPA and DPA levels in Italians is that the synthesis of both these FAs from ALA is partly inhibited ${ }^{24,25}$. This is in line with their high AA levels. There is indeed competition between n-3 and $n-6$ for the respective synthesis of EPA and AA, and low ALA is considered a factor for the preferred synthesis of AA from LA instead of EPAfrom ALA ${ }^{24,25}$.

When the whole dietary habits were taken into account through RRR-analysis, fish intake was a significant food component in the model explaining variations in RBC n-3 but not in the model explaining variations in plasma $n-3$. However, the variation in $n-3$ content of different species of fish and "farmed" or "wild" ones ${ }^{13,14,26}$ could not be considered in the present analysis. Only the total intake of fishes was taken in account. By this way the inside food group variation in EPA and DHA content could not be expressed.

In contrast, other food groups belonging to the two dietary patterns, such as cabbages, root vegetables, chocolate, coffee and tea, were significantly associated with variations in both plasma and RBC n-3, although these foods do not contain significant amounts of $n-3$. However, they contain high amounts of antioxidant vitamins and phytochemicals, which were shown to increase plasma and RBC n-3 in both human and animal studies ${ }^{27-29}$.

Some of the above mentioned food group consumption such as cabbages and vegetables is associated with vegetable oils intake, at high content of $n-3 F A$, as an internationally typical cooking and serving habit. This may overestimate their high contribution in dietary pattern extraction.

Further studies are needed to examine whether the findings reported here for Italians also apply to some other Mediterranean populations.

Despite the importance of the present work limitations still exist. The cross-sectional design of this study does not enable determination of causality. This study was not aimed at investigating biological mechanisms and further studies are necessary to investigate at which level(s) the foods identified in the two dietary patterns interfere with the metabolism of $n-3$ and $\mathrm{n}-6$ FA. Furthermore, the FFQ used in this study does not allow precise evaluation of each specific n-3 and n-6 FA and the kind of fish eaten, as fatty vs. non-fatty fish. Finally, possible errors by misreporting in the evaluation of the dietary habits of each individual should be acknowledged.

Concluding, a holistic approach in dietary analysis that takes into account FAs levels may contribute to public health promotion strategies. The healthy dietary pattern adherence should be placed as a major priority on the agenda of modern tactics for applying preventive medicine through nutrition.

\section{ACKNOWLEDGEMENTS}

The study was supported by European Union grant no QLK1-2000-00100.

The European Collaborative Group of the IMMIDIET Project is listed in the supplementary material. 


\section{REFERENCES}

1. Hoffman K, Schulze MB, Schienkiewitz A, Nöthlings U, Boeing H. Application of a new statistical method to derive dietary patterns in nutritional epidemiology. Am J Epidemiol 2004;159(10):935-944.

2. De Caterina R. N-3 fatty acids in cardiovascular disease. $\mathrm{N}$ Engl J Med 2011;364(25):2439-2450.

3. deLorgeril M, Salen P, Defaye P, Rabaeus M. Recent findings on the health effects of omega-3 fatty acids and statins, and their interactions: do statins inhibit omega3?BMC Med 2013:11:5.

4. Harris WS, Von Schacky C. The Omega-3 Index: a new risk factor for death from coronary heart disease? Prev Med 2004;39(1):212-220.

5. Samieri C, Maillard P, Crivello F, Proust-Lima C, Peuchant E, Helmer C, Amieva H, Allard M, Dartigues JF, Cunnane SC, et al.Plasma long-chain omega-3 fatty acids and atrophy of the medial temporal lobe. Neurology 2012;79(7):642-650.

6. Albert CM, Campos H, Stampfer MJ, Ridker PM, Manson JE, Willett WC, Ma J.Blood levels of long-chain $\mathrm{n}-3$ fatty acids and the risk of sudden death. $\mathrm{N}$ Engl $\mathrm{J}$ Med 2002;346(15):1113-1118

7. lacoviello L, Arnout J, Buntinx F, Cappuccio FP, Dagnelie PC, de Lorgeril M, Dirckx C, Donati MB, Krogh V, Siani A; European Collaborative Group of the IMMIDIET Project.Dietary habit profile in European communities with different risk of myocardial infarction: the impact of migration as a model of gene-environment interaction. The IMMIDIET Study. NutrMetabCardiovasc Dis 2001;11(4 Suppl):122-12b 6.

8. deLorgeril M, Salen P, Martin JL, Monjaud I, Delaye J, Mamelle N. Mediterranean diet, traditional risk factors, and the rate of cardiovascular complications after myocardial infarction: final report of the Lyon Diet Heart Study. Circulation 1999;99(6):779-785.

9. Cappuccio FP, Strazzullo P, FarinaroE,Trevisan M. Uric acid metabolism and tubular sodium handling: Results from a population-based study. JAMA 1993;270(3):354359.

10. Slimani N, Kaaks R, Ferrari P, Casagrande C, Clavel-Chapelon F, Lotze G, Kroke A, Trichopoulos D, Trichopoulou A, Lauria C,et al. European Prospective Investigation into Cancer and Nutrition (EPIC) calibration study: rationale, design and population characteristics. Public Health Nutr 2002;5(6B):1125-1145.

11. vanDongen MC, Lentjes MA, Wijckmans NE, Dirckx C, Lemaître $D$, Achten W, Celis $\mathrm{M}$, Sieri S, Arnout J, Buntinx $\mathrm{F}$, et al.Validation of a food-frequency questionnaire for Flemish and Italian-native subjects in Belgium: The IMMIDIET study. Nutrition 2011;27(3):302-309.

12. Pala V, Sieri S, Palli D, Salvini S, Berrino F, Bellegotti M, Frasca G, Tumino R, Sacerdote C, Fiorini $\mathrm{L}$, et al.Diet in the Italian EPIC cohorts: presentation of data and methodological issues. Tumori 2003;89(6):594-607.

13. McCance RA, Widdowson EM. The composition of foods. 5th ed. Cambridge, United Kingdom: The Royal Society of Chemistry and Ministry of Agriculture, Fisheries and Food; 1991.

14. Salvini S, Parpinel M, Gnagnarella P, Maisonnneuve P, Turrini A. BancaDati di ComposizionedegliAlimenti per StudiEpidemiologici in Italia. Milan, Italy: IstitutoEuropeo di Oncologia; 1998.

15. Stichting NEVO. NEVO: Dutchfooddatabase 2001 (NEVO-tabel: NederlandsVoedingsstoffenbestand 2001). Den Haag: Voedingscentrum; 2001 
16. Seeuws $\mathrm{C}$, et al. Belgian Food Composition Table (BelgischeVoedingsmiddelentabel); 3rd ed. Brussel: NUBEL v.z.w. (NutriëntenBelgië); 1999.

17. O'Brien E, Waeber B, Parati G, Staessen J, Myers MG. Blood pressure measuring devices: recommendations of the European Society of Hypertension. BMJ 2001;322(7285):531-536.

18. Friedewald WT, Levy RI, Fredrickson DS. Estimation of the concentration of lowdensity lipoprotein cholesterol in plasma without use of the preparative ultracentrifuge. ClinChem 1972;18(6):499-502.

19. Hodson L, Skeaff CM, Wallace AJ, Arribas GL. Stability of plasma and erythrocyte fatty acid composition during cold storage. ClinChem 2002;321(1-2):63-67.

20. Masson S, Marchioli R, Mozaffarian D, Bernasconi R, Milani V, Dragani L, Tacconi M, Marfisi RM, Borgese L, Cirrincione V, et al. Plasma n-3 polyunsaturated fatty acids in chronic heart failure in the GISSI-Heart Failure Trial: relation with fish intake, circulating biomarkers, and mortality. Am Heart J2013;165(2):208-215.

21. Khaw KT, Friesen MD, Riboli E, Luben R, Wareham N. Plasma phospholipid fatty acid concentration and incident coronary heart disease in men and women: the EPICNorfolk prospective study. PLoS Med 2012;9(7):e1001255.

22. Kuehl FA Jr, Egan RW. Prostaglandins, arachidonic acid, and inflammation. Science 1980;210(4473):978-984.

23. Chowdhury R, Stevens S, Gorman D, Pan A, Warnakula S, Chowdhury S, Ward H, Johnson L, Crowe F, Hu FB, Franco OH. Association between fish consumption, long chain omega 3 fatty acids, and risk of cerebrovascular disease: systematic review and meta-analysis. BMJ 2012;345:e6698.

24. Burdge GC, Wootton SA. Conversion of alpha-linolenic acid to eicosapentaenoic, docosapentaenoic and docosahexaenoic acids in young women. $\mathrm{Br} J$ Nutr 2002;88(4):411-420.

25. Burdge GC, Jones AE, Wootton SA. Eicosapentaenoic and docosapentaenoic acids are the principal products of alpha-linolenic acid metabolism in young men. $\mathrm{Br} \mathrm{J}$ Nutr 2002;88(4):355-363.

26. M.A. Hossain. Fish as Source of n-3 Polyunsaturated Fatty Acids (PUFAs), Which One is Better-Farmed or Wild? Advance Journal of Food Science and Technology 3(6): 455-466, 2011.

27. deLorgeril M, Salen P, Martin JL, Boucher F, de Leiris J. Interactions of wine drinking with omega-3 fatty acids in coronary heart disease patients: a fish-like effect of moderate wine drinking. Am Heart J 2008;155(1):175-181.

28. di Giuseppe R, de Lorgeril M, Salen P, Laporte F, Di Castelnuovo A, Krogh V, Siani A, Arnout J, Cappuccio FP, van Dongen $M$, et al.Alcohol consumption and n-3 polyunsaturated fatty acids in healthy men and women from 3 European populations. Am J ClinNutr 2009;89(1):354-362.

29. Toufektsian MC, Salen P, Laporte F, Tonelli C, de Lorgeril M. Dietary flavonoids increase plasma very long-chain (n-3) fatty acids in rats. J Nutr 2011;141(1):37-41. 


\begin{tabular}{|c|c|c|c|c|}
\hline & \multicolumn{3}{|c|}{ Country of residence } & \multirow[b]{2}{*}{$P^{b}$} \\
\hline & Italy $(n=542)$ & Belgium $(n=536)$ & UK $(n=526)$ & \\
\hline \multicolumn{5}{|l|}{ FAs ( $\%$ of total FAs) ${ }^{a}$} \\
\hline \multicolumn{5}{|l|}{ Saturated } \\
\hline 14:0 (myristic) & $1.20(0.83-1.61)^{1}$ & $1.06(0.76-1.39)^{1,2}$ & $1.17(0.87-1.55)^{2}$ & $<0.001$ \\
\hline 16:0 (palmitic) & $22.0(20.7-23.7)^{1}$ & $21.6(20.2-23.1)^{1}$ & $21.7(20.5-23.2)$ & $<0.001$ \\
\hline 18:0 (stearic) & $5.67(5.13-6.22)$ & $5.85(5.25-6.48)$ & $5.72(5.26-6.24)$ & 0.004 \\
\hline 20:0 (arachidic) & $0.04(0.03-0.05)^{1}$ & $0.05(0.04-0.07)^{1,2}$ & $0.04(0.03-0.05)^{2}$ & $<0.001$ \\
\hline \multicolumn{5}{|l|}{ Unsaturated } \\
\hline $18: 3 n-3(A L A)$ & $0.44(0.36-0.56)^{1,2}$ & $0.83(0.66-1.01)^{1,3}$ & $0.89(0.73-1.06)^{2,3}$ & $<0.001$ \\
\hline $20: 5 n-3(E P A)$ & $0.60(0.46-0.82)^{1,2}$ & $0.92(0.69-1.25)^{1,3}$ & $1.27(0.99-1.65)^{2,3}$ & $<0.001$ \\
\hline $22: 5 n-3$ (DPA) & $0.41(0.34-0.51)^{1,2}$ & $0.54(0.45-0.63)^{1,3}$ & $0.63(0.55-0.73)^{2,3}$ & $<0.001$ \\
\hline $22: 6 n-3(D H A)$ & $1.88(1.51-2.26)^{1,2}$ & $2.06(1.72-2.53)^{1,3}$ & $2.25(1.79-2.94)^{2,3}$ & 0.003 \\
\hline $18: 2 n-6(L A)$ & $29.8(26.4-33.0)^{1,2}$ & $33.5(30.0-36.7)^{1,3}$ & $31.3(27.7-34.0)^{2,3}$ & $<0.001$ \\
\hline $18: 3 n-6(G L A)$ & $0.59(0.48-0.77)^{1,2}$ & $0.50(0.39-0.64)^{1}$ & $0.54(0.42-0.67)^{2}$ & $<0.001$ \\
\hline $20: 2 n-6(E D A)$ & $0.18(0.15-0.23)^{1}$ & $0.23(0.20-0.26)^{1,2}$ & $0.20(0.17-0.23)^{2}$ & $<0.001$ \\
\hline $20: 3 n-6(D G L A)$ & $1.67(1.45-1.92)^{1,2}$ & $1.46(1.22-1.70)^{1}$ & $1.49(1.31-1.73)^{2}$ & $<0.001$ \\
\hline $20: 4 n-6(A A)$ & $8.23(6.95-9.26)^{1,2}$ & $7.45(6.44-8.46)^{1,3}$ & $6.82(6.00-7.73)^{2,3}$ & $<0.001$ \\
\hline $22: 4 n-6$ (ardenic) & $0.21(0.18-0.25)^{1}$ & $0.21(0.17-0.25)^{2}$ & $0.18(0.15-0.23)^{1,2}$ & $<0.001$ \\
\hline $16: 1 n-7$ (palmitoleic) & $2.13(1.69-2.92)^{1}$ & $2.13(1.66-2.88)^{2}$ & $2.70(2.16-3.37)^{1,2}$ & $<0.001$ \\
\hline $18: 1 n-7$ (cis-vaccenic) & $1.63(1.43-1.90)^{1}$ & $1.56(1.38-1.75)^{1,2}$ & $1.64(1.44-1.91)^{2}$ & $<0.001$ \\
\hline $18: 1 n-9$ (oleic) & $21.8(19.7-24.0)^{1,2}$ & $18.4(16.8-20.4)^{1,3}$ & $19.9(18.1-21.7)^{2,3}$ & $<0.001$ \\
\hline $20: 3 n-9$ (mead) & $0.14(0.11-0.19)^{1,2}$ & $0.10(0.08-0.13)^{1,3}$ & $0.12(0.09-0.16)^{2,3}$ & $<0.001$ \\
\hline
\end{tabular}

\footnotetext{
${ }^{a}$ Plasma FAs were presented as median $\left(1^{\text {st }}-3^{\text {rd }}\right.$ quartiles $)$ because their distributions were skewed.

${ }^{\mathrm{b}} \mathrm{P}$-values derived through one-way ANOVA F-tests for country differences on log-transformed plasma FAs levels.

$1,2,3$ Indicated pairs of countries with significantly different levels on each FA according to Bonferroni comparisons. Bonferroni correction for multiple comparisons (17 tests and significance level a=0.05) yield a corrected $a^{\prime}=0.003$ which indicated significant differences at the aforementioned univariate analysis.
}

\begin{tabular}{|c|c|c|c|c|}
\hline & \multicolumn{3}{|c|}{ Country of residence } & \multirow[b]{2}{*}{$\mathrm{P}^{\mathrm{b}}$} \\
\hline & Italy $(n=542)$ & Belgium $(n=536)$ & UK $(n=526)$ & \\
\hline \multicolumn{5}{|l|}{ FAs (\% of total FAs) ${ }^{a}$} \\
\hline \multicolumn{5}{|l|}{ Saturated } \\
\hline 16:0 (palmitic) & $21.4(20.6-22.1)^{1,2}$ & $21.0(20.4-21.9)^{1,3}$ & $22.1(21.2-23.2)^{2,3}$ & $<0.001$ \\
\hline $18: 0$ (stearic) & $15.8(15.0-16.6)$ & $16.0(15.1-16.8)$ & $16.1(15.2-16.9)$ & 0.006 \\
\hline 20:0 (arachidic) & $0.08(0.07-0.09)$ & $0.09(0.08-0.10)$ & $0.09(0.07-0.10)$ & 0.48 \\
\hline \multicolumn{5}{|l|}{ Unsaturated } \\
\hline $18: 3 n-3(A L A)$ & $0.09(0.07-0.11)^{1,2}$ & $0.19(0.16-0.23)^{1}$ & $0.19(0.16-0.23)^{2}$ & $<0.001$ \\
\hline $20: 5 n-3$ (EPA) & $0.63(0.52-0.78)^{1,2}$ & $1.00(0.82-1.27)^{1,3}$ & $1.21(0.97-1.57)^{2,3}$ & $<0.001$ \\
\hline $22: 5 n-3$ (DPA) & $2.54(2.92-2.24)^{1,2}$ & $3.09(2.83-3.40)^{1,3}$ & $3.30(2.92-3.65)^{2,3}$ & $<0.001$ \\
\hline $22: 6 n-3(D H A)$ & $6.16(5.35-6.91)$ & $6.40(5.49-7.22)^{1}$ & $6.15(5.03-7.27)^{1}$ & $<0.001$ \\
\hline $18: 2 n-6(L A)$ & $10.8(10.1-11.9)^{1,2}$ & $12.4(11.6-13.4)^{1,3}$ & $11.8(10.9-12.7)^{2,3}$ & $<0.001$ \\
\hline $18: 3 n-6(G L A)$ & $0.06(0.05-0.07)$ & $0.06(0.04-0.08)$ & $0.06(0.05-0.08)$ & 0.03 \\
\hline $20: 2 n-6(E D A)$ & $0.22(0.20-0.25)^{1}$ & $0.26(0.23-0.30)^{1,2}$ & $0.22(0.20-0.26)^{2}$ & $<0.001$ \\
\hline $20: 3 n-6$ (DGLA) & $1.91(1.67-2.19)^{1,2}$ & $1.77(1.57-2.03)^{1}$ & $1.80(1.56-2.06)^{2}$ & $<0.001$ \\
\hline $20: 4 n-6(A A)$ & $19.3(18.2-20.4)^{1,2}$ & $18.3(17.3-19.3)^{1,3}$ & $16.6(15.3-17.8)^{2,3}$ & $<0.001$ \\
\hline $22: 4 n-6$ (ardenic) & $3.66(3.32-4.05)^{1,2}$ & $3.30(2.90-3.73)^{1,3}$ & $2.76(2.37-3.20)^{2,3}$ & $<0.001$ \\
\hline $16: 1 n-7$ (palmitoleic) & $0.30(0.23-0.41)^{1,2}$ & $0.35(0.27-0.46)^{1,3}$ & $0.40(0.31-0.53)^{2,3}$ & $<0.001$ \\
\hline 18:1n-7(cis-vaccenic) & $1.65(1.41-1.88)^{1,2}$ & $1.24(1.13-1.49)^{1,3}$ & $1.48(1.22-1.81)^{2,3}$ & $<0.001$ \\
\hline 18:1n-9 (oleic) & $14.4(13.5-15.2)^{1}$ & $13.4(12.7-14.1)^{1,2}$ & $14.5(13.8-15.2)^{2}$ & $<0.001$ \\
\hline $20: 3 n-9$ (mead) & $0.08(0.06-0.10)^{1,2}$ & $0.06(0.05-0.07)^{1,3}$ & $0.07(0.05-0.09)^{2,3}$ & $<0.001$ \\
\hline Omega-3 Index (EPA +DHA) & $6.82(5.93-7.71)^{1,2}$ & $7.44(6.33-8.48)^{1}$ & $7.42(6.06-8.72)^{2}$ & $<0.001$ \\
\hline \multicolumn{5}{|c|}{${ }^{\mathrm{a}} \mathrm{RBC}$ FAs were presented as median $\left(1^{\text {st }}-3^{\text {rd }}\right.$ quartiles) because their distributions were skewed. } \\
\hline
\end{tabular}




\begin{tabular}{|c|c|c|}
\hline \multirow[b]{2}{*}{$N=1604$} & \multicolumn{2}{|c|}{ Factor loadings } \\
\hline & Model $1^{a}$ & Model $2^{b}$ \\
\hline \multicolumn{3}{|l|}{ Food product groups (g/day) } \\
\hline \multicolumn{3}{|l|}{ Starches (Bread, potatoes, legumes) } \\
\hline Cabbages \& root vegetables & 0.48 & 0.42 \\
\hline \multicolumn{3}{|l|}{ Leafy vegetables and tomatoes } \\
\hline \multicolumn{3}{|l|}{ Fruits \& fruit or vegetable juices } \\
\hline Diaries & 0.29 & \\
\hline Cheese & -0.22 & \\
\hline Pasta, rice & -0.31 & -0.28 \\
\hline Red meat \& products & -0.27 & -0.24 \\
\hline \multicolumn{3}{|l|}{ White meat \& products } \\
\hline Fishes & & 0.49 \\
\hline Mollusks & & 0.25 \\
\hline Vegetable oils & 0.21 & 0.20 \\
\hline Olive oil & -0.29 & -0.26 \\
\hline Nuts and seeds & & \\
\hline \multicolumn{3}{|l|}{ Fats \& pizza } \\
\hline \multicolumn{3}{|l|}{ Sweets \& sugars } \\
\hline \multicolumn{3}{|l|}{ Snacks } \\
\hline Chocolate, coffee \& tea & 0.40 & 0.27 \\
\hline Mayonnaise & 0.20 & 0.22 \\
\hline Alcoholic drinks & & \\
\hline Alcohol contained in all food groups & & \\
\hline
\end{tabular}

\footnotetext{
${ }^{a}$ Model 1 derived through RRR-analysis with main outcome the log-transformed total plasma n-3 FAs. Factor loadings with absolute values lower than 0.20 were not presented for simplicity.

${ }^{b}$ Model 2 derived through RRR-analysis with main outcome the log-transformed total RBC n-3 FAs. Factor loadings with absolute values lower than 0.20 were not presented for simplicity.
}

\begin{tabular}{|c|c|c|c|c|}
\hline \multirow[b]{2}{*}{$N=1604$} & \multicolumn{2}{|c|}{ Total plasma $\mathrm{n}-3$} & \multicolumn{2}{|c|}{ Total RBC n-3 } \\
\hline & Spearman's rho & $P$-value ${ }^{a}$ & Spearman's rho & $P$-value ${ }^{b}$ \\
\hline \multicolumn{5}{|l|}{ Food groups (g/day) } \\
\hline Cabbages \& root vegetables & 0.45 & $<0.001$ & 0.24 & $<0.001$ \\
\hline Diaries & 0.28 & $<0.001$ & 0.12 & $<0.001$ \\
\hline Cheese & -0.22 & $<0.001$ & -0.04 & 0.07 \\
\hline Pasta \& rice & -0.27 & $<0.001$ & -0.13 & $<0.001$ \\
\hline Red meat \& products & -0.28 & $<0.001$ & -0.15 & $<0.001$ \\
\hline Fishes & 0.10 & $<0.001$ & 0.25 & $<0.001$ \\
\hline Mollusks & 0.10 & 0.001 & 0.13 & $<0.001$ \\
\hline Vegetable oils & 0.30 & $<0.001$ & 0.14 & $<0.001$ \\
\hline Olive oil & -0.28 & $<0.001$ & -0.13 & $<0.001$ \\
\hline Chocolate, coffee \& tea & 0.42 & $<0.001$ & 0.20 & $<0.001$ \\
\hline Mayonnaise & 0.30 & $<0.001$ & 0.18 & $<0.001$ \\
\hline
\end{tabular}

${ }^{\mathrm{a}} \mathrm{P}$-value derived through Spearman's rho examining the association between food group consumption and total plasma n-3 FAs.

${ }^{\mathrm{b}} \mathrm{P}$-value derived through Spearman's rho examining the association between food group consumption and total RBC n-3 FAs. 


\begin{tabular}{|c|c|c|c|c|}
\hline \multirow[b]{2}{*}{$N=1604$} & \multicolumn{3}{|c|}{ Country of residence } & \multirow[b]{2}{*}{$P^{a}$} \\
\hline & Italy $(n=542)$ & Belgium $(n=536)$ & UK $(n=526)$ & \\
\hline RRR-score from patterns analysis $1^{b}$ & $-1.23(-1.70,-0.74)$ & $0.09(-0.43,0.56)$ & $1.07(0.50-1.65)$ & $<0.001$ \\
\hline RRR-score from patterns analysis $2^{b}$ & $-0.79(-1.28,-0.16)$ & $0.13(-0.42,0.72)$ & $0.36(-0.12,0.97)$ & $<0.001$ \\
\hline \multicolumn{5}{|l|}{ Food groups (g/day) } \\
\hline Starches (Bread, potatoes, legumes) & $169(112-251)$ & $326(251-412)$ & $198(139-265)$ & $<0.001$ \\
\hline Cabbages \& root vegetables & $40(26.3-69.0)$ & $95(69-132)$ & $165(107-224)$ & $<0.001$ \\
\hline Leafy vegetables and tomatoes & $125(85-167)$ & $83(116-154)$ & $103(68-151)$ & $<0.001$ \\
\hline Fruits \& fruit or vegetable juices & $365(248-530)$ & $226(126-398)$ & $243(142-381)$ & $<0.001$ \\
\hline Diaries & $125(14-224)$ & $98(26-208)$ & $285(351-507)$ & $<0.001$ \\
\hline Cheese & $43(28-68)$ & $49(24-80)$ & $17(5-27)$ & $<0.001$ \\
\hline Pasta \& rice & $71(44-101)$ & $44(29-64)$ & $30(18-56)$ & $<0.001$ \\
\hline Red meat \& products & $106(72-154)$ & $148(105-209)$ & $45(29-80)$ & $<0.001$ \\
\hline White meat \& products & $45(29-62)$ & $52(35-69)$ & $50(25-59)$ & $<0.001$ \\
\hline Fishes & $26(16-38)$ & $22(14-35)$ & $11(5-22)$ & $<0.001$ \\
\hline Mollusks & $7.6(3.6-16.2)$ & $10.5(0-18.2)$ & $6.7(3.8-12.0)$ & 0.10 \\
\hline Vegetable oils & $0.8(0.4-0.8)$ & $19(9-36)$ & $15(4-28)$ & $<0.001$ \\
\hline Olive oil & $25(19-33)$ & $3.6(2.3-5.8)$ & $0(0-22)$ & $<0.001$ \\
\hline Nuts \& seeds & $0.2(0.2-0.7)$ & $1.8(0-7.0)$ & $1.1(0.7-3.4)$ & $<0.001$ \\
\hline Fats \& pizza & $47(33-67)$ & $49(19-74)$ & $23(11-36)$ & $<0.001$ \\
\hline Sweets \& sugars & $96(49-177)$ & $230(120-486)$ & $101(47-198)$ & $<0.001$ \\
\hline Snacks & $14(5-30)$ & $14(7-25)$ & $3(1-10)$ & $<0.001$ \\
\hline Chocolate, coffee \& tea & $130(90-195)$ & $671(391-1062)$ & $964(681-1358)$ & $<0.001$ \\
\hline Mayonnaise & $0.1(0-0.5)$ & $7.0(3.5-10.5)$ & $2.6(1.0-6.9)$ & $<0.001$ \\
\hline Alcoholic drinks & $84(7-258)$ & $134(35-346)$ & $94(19-240)$ & $<0.001$ \\
\hline Alcohol contained in all food groups & $6.0(0.3-23.8)$ & $8.6(2.6-23.5)$ & $12.7(10.2-14.9)$ & $<0.001$ \\
\hline Total energy intake (Kcal/d) & $2324(1940-2852)$ & $2644(2210-3291)$ & $2114(1756-2603)$ & $<0.001$ \\
\hline Total protein $(\% \mathrm{Kcal} / \mathrm{d})$ & $15.9(14.6-17.3)$ & $17.0(15.6-18.5)$ & $14.5(13.1-16.0)$ & $<0.001$ \\
\hline Total carbohydrates (\% Kcal/d) & $49.3(44.0-54.3)$ & $42.4(39.0-46.7)$ & $47.8(43.9-52.1)$ & $<0.001$ \\
\hline Total fibers $(\mathrm{g} / \mathrm{d})$ & $16(13.2-19.8)$ & $22.8(18.7-27.6)$ & $14.1(11.0-17.5)$ & $<0.001$ \\
\hline Total fat $(\% \mathrm{Kcal} / \mathrm{d})$ & $33.6(30.5-37.6)$ & $36.6(33.1-40.1)$ & $36.9(33.4-40.5)$ & $<0.001$ \\
\hline Total saturated lipids (\%Kcal/d) & $11.8(10.2-13.2)$ & $14.4(12.9-16.3)$ & $11.9(10.0-14.0)$ & $<0.001$ \\
\hline Total monounsaturated lipids (\%Kcal/d) & $16.2(14.2-18.2)$ & $12.2(11.0-13.4)$ & $13.1(10.8-15.3)$ & $<0.001$ \\
\hline Total polyunsaturated lipids (\%Kcal/d) & $3.7(3.3-4.1)$ & $6.6(5.7-8.0)$ & $7.5(5.3-7.5)$ & $<0.001$ \\
\hline
\end{tabular}

${ }^{\mathrm{a} P}$-values derived through Kruskal-Wallis test for country differences.

${ }^{\mathrm{b}}$ Each of the RRR-scores 1 and 2 was derived through RRR-analysis with main outcome the log-transformed total plasma and RBC n-3 FAs, correspondingly. 


\section{Supplementary file}

Clinical and anthropometric characteristics of study participants according to country of residence were presented in Supplementary Table. Italians were slightly younger as compared to Belgians and English, had higher levels of BMI, SBP, DBP and triglycerides but lower serum levels of LDL-cholesterol and fasting glucose.

The trend of Italians to have lower plasma n-3 than the participants of the other two populations is illustrated in Figure $S 1$ where plasma n-3 distributions for the Italian subpopulation are always shifted more to the left than those for the Belgian and UK. In addition, UK levels seemed the highest of the three populations in most cases. In particular, for the Italian subpopulation over $50 \%$ of all $\mathrm{n}-3$ distributions was under the median for the total study population.

Similarly the trend of Italians to have lower n-3 RBC compared with the two other populations is illustrated Figure S2, where RBC n-3 FA distributions for the Italian subpopulation were always shifted more to the left than for the Belgian and UK. More accurately, for the Italian sub-population over $50 \%$ of all n-3 RBC distributions was under the median of the total study population. An exception was RBC DHA level that did not show this trend.

\begin{tabular}{|c|c|c|c|c|}
\hline & \multicolumn{3}{|c|}{ Country of residence } & \multirow[b]{2}{*}{$\mathrm{P}^{\mathrm{b}}$} \\
\hline & Italy $(n=542)$ & Belgium $(n=536)$ & UK $(n=526)$ & \\
\hline Age (years) & $44.4(7.1)^{1,2}$ & $46.2(8.5)^{1}$ & $48.0(7.5)^{2}$ & $<0.001$ \\
\hline BMI $\left(\mathrm{Kg} / \mathrm{m}^{2}\right)$ & $27.2(4.4)^{1,2}$ & $26.1(4.3)^{1,3}$ & $26.4(4.3)^{2,3}$ & $<0.001$ \\
\hline $\mathrm{SBP}(\mathrm{mmHg})$ & $126(18)^{1}$ & $123(16)^{2}$ & $120(18)^{1,2}$ & $<0.001$ \\
\hline $\mathrm{DBP}(\mathrm{mmHg})$ & $80(11)^{1}$ & $78(10)$ & $77(10)^{3}$ & 0.002 \\
\hline Total cholesterol (mg/dl) & $215(37)^{1}$ & $227(39)^{1,2}$ & $215(39)^{2}$ & $<0.001$ \\
\hline LDL-cholesterol (mg/dl) & $138(33)^{1}$ & $149(36)^{1,2}$ & $141(35)^{2}$ & $<0.001$ \\
\hline HDL-cholesterol (mg/dl) & $50(13)^{1}$ & $56(15)^{1,2}$ & $53(14)^{2}$ & $<0.001$ \\
\hline Triglycerides (mg/dl) & $132(94)^{1,2}$ & $110(68)^{1}$ & $109(66)^{2}$ & $<0.001$ \\
\hline Fasting glucose (mg/dl) & $76.1(19.6)^{1,2}$ & $78.7(11.4)^{1,3}$ & $88.9(13.0)^{2,3}$ & $<0.001$ \\
\hline
\end{tabular}

${ }^{a}$ Continuous data are presented as mean (standard deviation).

${ }^{\mathrm{b}} \mathrm{P}$-values derived through one-way ANOVA F-tests for country differences normally distributes continuous characteristics.

\section{Legend to the Figures}

Figure S1. Distribution of plasma n-3 FAs in Italy, Belgium and UK

Figure S2. Distribution of RBC n-3 FAs in Italy, Belgium and UK 
Figure S1.
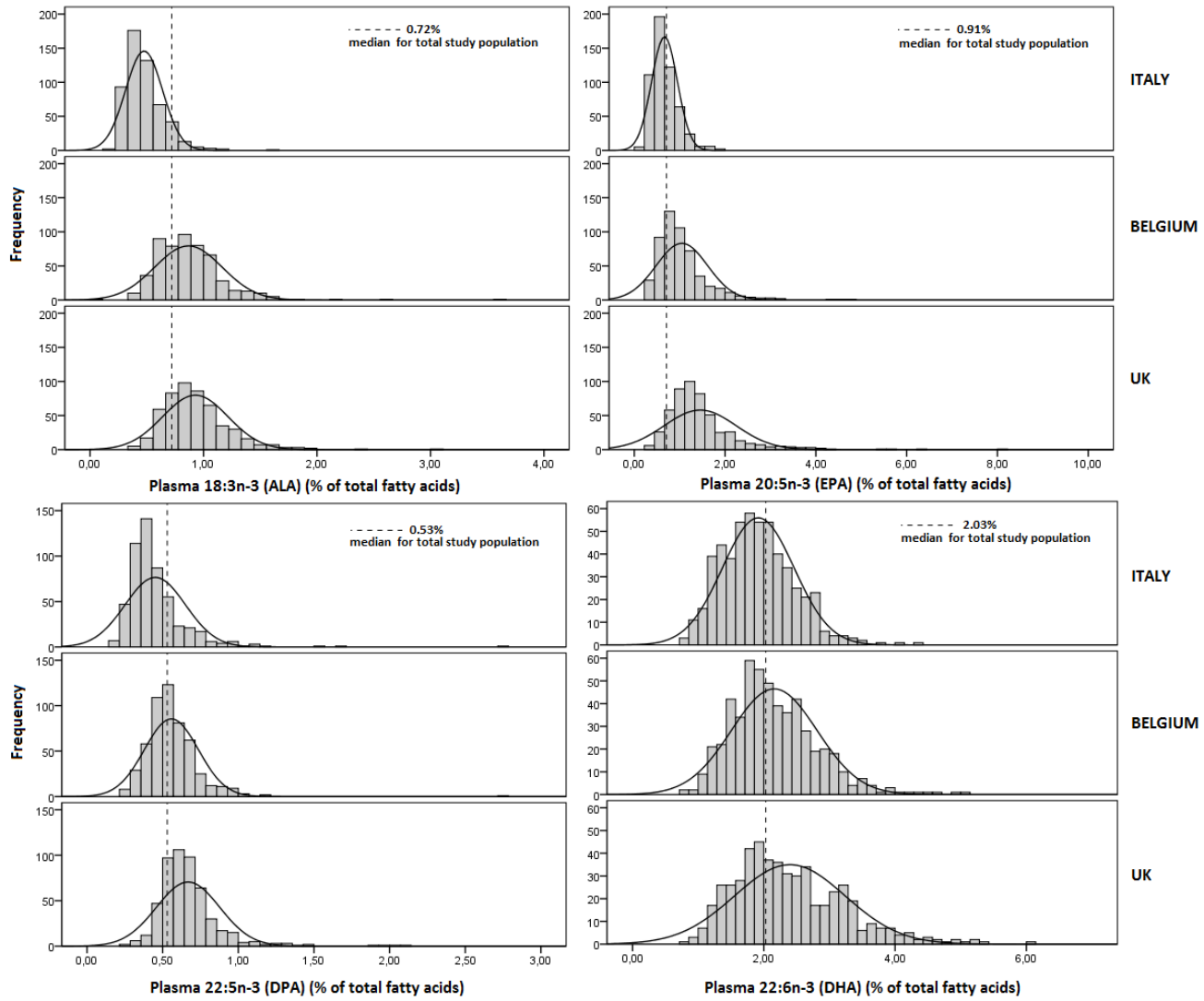

Figure S2.
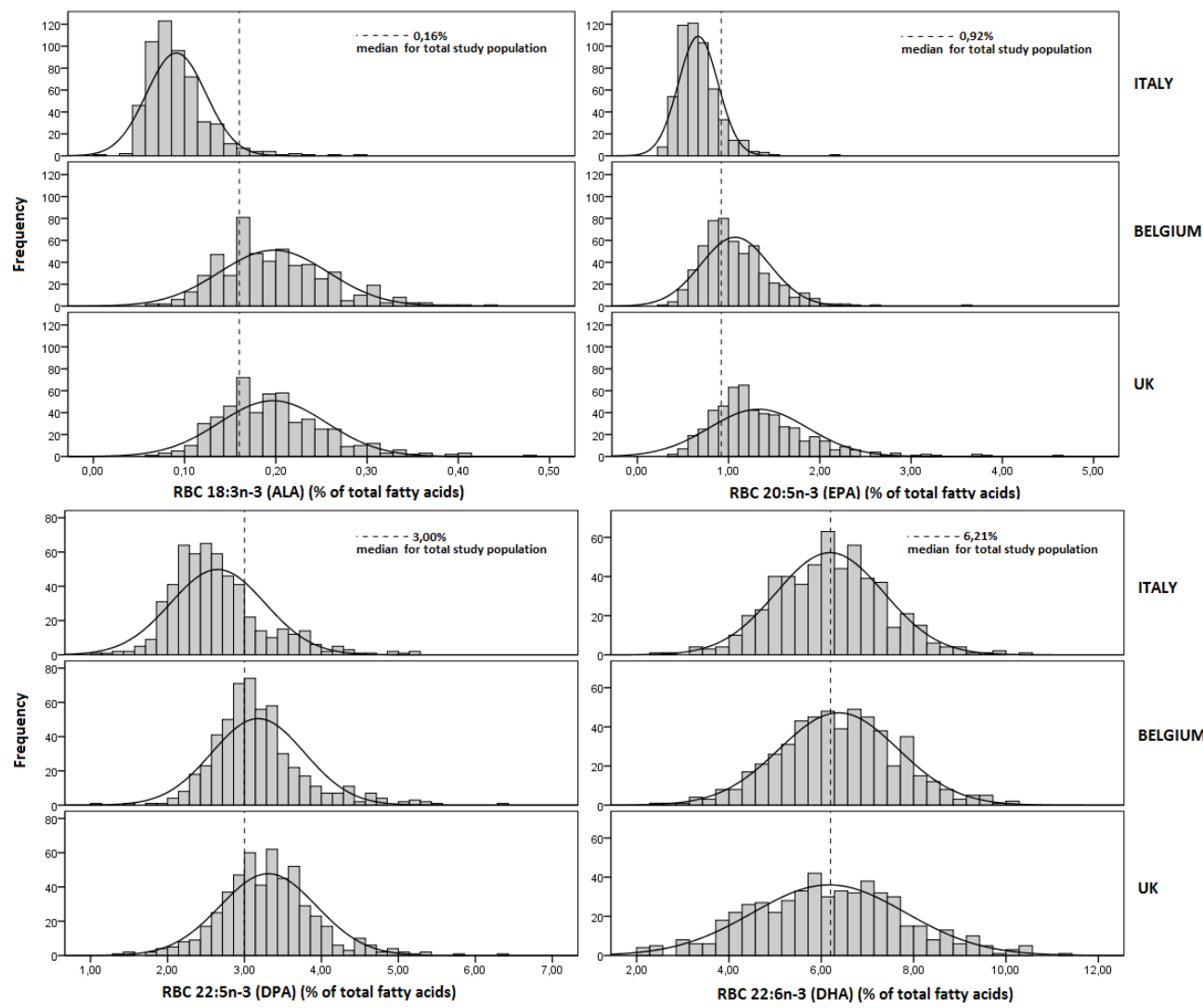


\section{European Collaborative Group of the IMMIDIET Project}

Project Co-ordinator: Licia lacoviello ${ }^{a}$

Scientific Committee: Jef Arnout, ${ }^{b}$ Frank Buntinx, ${ }^{c}$ Francesco P. Cappuccio, ${ }^{d}$ Pieter C. Dagnelie, ${ }^{e}$ Maria Benedetta Donati, ${ }^{a}$ Michel de Lorgeril, ${ }^{f}$ Vittorio Krogh, ${ }^{g}$ Alfonso Siani ${ }^{\text {h }}$

Co-ordinating secretariat: Carla Dirckx ${ }^{\mathrm{b}, \mathrm{c}}$

Data management and statistics: Augusto Di Castelnuovo ${ }^{a}$

Dietary assessment and analysis: Martien van Dongen ${ }^{e}$

Communication and dissemination: Americo Bonanni ${ }^{a}$

Recruitment: Carla Dirckx, ${ }^{\mathrm{b}, \mathrm{c}}$ Pit Rink, ${ }^{\mathrm{d}}$ Branislav Vohnout, ${ }^{\mathrm{a}}$ Francesco Zito ${ }^{\mathrm{a}}$

External advisory committee: Mario Mancini, Napoli, Italy; Antonia Trichopoulou, Athens, Greece

The IMMIDIET group, collaborative centres and associated investigators

a. Fondazione di Ricerca e Cura "Giovanni paolo II", Catholic University, Campobasso, Italy (Licia lacoviello, Francesco Zito, Augusto Di Castelnuovo, Americo Bonanni, Branislav Vohnout, Marco Olivieri, Amalia De Curtis, Agnieszka Pampuch, Maria Benedetta Donati, Giovanni de Gaetano)

b. Centre for Molecular and Vascular Biology, Katholieke Universiteit Leuven, Leuven, Belgium (Jef Arnout, Carla Dirckx, Ward Achten)

c. Department of General Practice, Katholieke Universiteit Leuven, Leuven, Belgium (Frank Buntinx, Carla Dirckx, Jan Heyrman)

d. Clinical Sciences Research Institute, Warwick Medical School, Coventry, United Kingdom (Francesco P. Cappuccio, Michelle A Miller); Division of Community Health Sciences, St George's, University of London, United Kingdom (Pit Rink, Sally C Dean, Clare Harper)

e. Department of Epidemiology, CAPHRI, Subdivision of Nutritional and Molecular Epidemiology, Maastricht University, Maastricht, The Netherlands (Pieter Dagnelie, Martien van Dongen, Dirk Lemaître)

f. Nutrition, Vieillissement et Maladies Cardiovasculaires (NVMCV), UFR de Médecine, Domaine de la Merci, 38056 La Tronche, France (Michel de Lorgeril)

g. Nutritional Epidemiology Unit, National Cancer Institute, Milan, Italy (Vittorio Krogh, Sabrina Sieri, Manuela Bellegotti, Daniela Del Sette Cerulli)

h. Unit of Epidemiogy \& Population Genetics, Institute of Food Sciences CNR, Avellino, Italy (Alfonso Siani, Gianvincenzo Barba, Paola Russo, Antonella Venezia) 


\section{Chapter 8. General Discussion}

The aim of this thesis was to examine the complex impact of dietary habits on major cardiovascular risk factors and other metabolic parameters of free-living populations, using different methodological approaches to dietary analysis, in order to get more integrated conclusions.

\section{A number of crucial issues have been addressed:}

- The holistic evaluation of antioxidant vitamins and phytochemicals intake through FAC-score: a dietary index.

The content in antioxidant vitamins and phytochemicals of a healthy diet such as the Mediterranean diet has long been recognized as beneficial for inflammation related illnesses such as atherosclerosis. Antioxidant vitamins and flavonoids contained in several foods were associated in epidemiological and clinical studies with a decrease in the oxidative stress caused by these risk factors.

In the context of the Moli-sani study a dietary index "FAC-score" was developed with main aim the evaluation of antioxidant food intake. In the sample of free-living apparently healthy men the choice of foods high rather than low in antioxidant content was associated with reduced blood pressure and inflammation.

The calculation of this score in the total population gives the opportunity to overall assess the antioxidant food intake as a serious aspect of a healthy diet.

- Dietary index construction as a methodological approach in dietary analysis that gives valuable conclusions about the association of diet with other than clinical CVD risk factors.

HRQL is considered as a valid predictor of mortality. FAC-score was studied in the context of Mediterranean pattern adherence for its role in establishing better quality of life for the Moli-sani population. 
HRQL was positively associated with Mediterranean pattern adherence, while this association disappeared after further adjustment for FAC-score. This indicates that the total antioxidant content of diet could independently explain this relationship.

Conclusions supported that a scoring system in dietary assessment could also be associated with other CVD disease risk factors, not necessarily clinically evaluable.

- Mushroom and selenium intake in association with glucose levels: the evaluation of food and nutrient consumption as a methodology in dietary analysis.

During the study of FAC-score in the Moli-sani population, it was observed that mushroom, as a food group, could be considered as good source of selenium, an important antioxidant component of the diet. The role of selenium on glucose levels is somehow controversial. Thus a second analysis conducted in the Moli-sani population aimed at evaluating the association of mushroom consumption and dietary selenium intake with blood glucose levels.

Both mushroom intake and dietary selenium were independently and positively associated with blood glucose in the multivariate analysis suggesting that they might independently influence the risk of diabetes.

Such analyses concerning food group consumption (through mushroom intake) and nutrient intake (through dietary selenium) explored in a different way the role of antioxidants on CVD risk.

The conclusions stress the importance of studying antioxidants in relation to CVD biomarkers at a different level than the holistic approach of FAC-score, through food and nutrient intake. This can help to better evaluate the importance of such food groups for public health perspectives.

- Folate intake and dietary patterns in association with folate blood levels. Should dietary pattern analysis be a useful tool in the study of human diet and CVD related biomarkers? 
Folate status has been negatively associated with cerebrovascular disease, an important category of CVD. In the context of the IMMIDIET study possible differences in folate status in two EU Countries (Italy and England) were assessed in relation to folate intake, dietary pattern adherence and/or other lifestyle parameter.

Results indicated an inadequate dietary folate intake and subsequent serum levels in Southern-Italian subjects, compared to English ones. In addition, an increase in dietary folate intake was associated with an increase in folate serum levels in the Italian and English population.

RRR-analysis indicated healthy patterns including high intake of vegetables, potatoes, bread, fruits, legumes, white meat, breakfast cereals and dairy that was positively associated with folate serum levels in both countries. However, these patterns were less adhered to by Italians.

The conclusions stress the importance of an adequate intake of folate through natural food sources in establishing adequate serum levels, an important aspect for cerebrovascular disease.

- Is the study of single supplemental nutrient intake through clinical trials enough to establish an association of human diet with CVD related biomarkers? Folate as an example.

A further study on the supplemental intake of folate as a single nutrient was performed using data from a randomized, double blind clinical trial. Major aim was to compare the efficacy of a diet particularly rich in natural folate compared with that of two different protocols of folic acid pharmacological supplementation, in subjects with "moderate" hyperhomocysteinemia.

Results indicated that supplementation with natural folate-rich foods, folic acid or [6S]5methyltetrahydrofolate (5-MTHF) reached a similar reduction in homocysteine concentrations. 
This study confirmed that increasing folate levels by consuming food naturally rich in folate is a valuable approach to decrease homocysteine levels (a risk factor for cerebrovascular disease, in subjects with mild hyperhocysteinemia). The increased bioavailability and bioefficacy of folate in natural food sources may lead to these results.

The study of single supplemental nutrient intake through clinical trials may not be enough in establishing associations between diet and CVD related biomarkers.

- Dietary patterns and blood and tissue fatty acids. A second example about the usefulness of dietary pattern analysis as tool in the study of human diet and CVD related biomarkers.

Growing evidence suggests that blood FAs are major biomarkers/predictors and even causal factors in many diseases, in particular cardiovascular disease. Differences in blood FAs profile among populations with different lifestyle have partly been attributed to differences in food intake. This analysis aimed at evaluating the main plasma and red blood cell (RBC) FAs in three European populations and assessing the role of dietary patterns in explaining variations in their levels. The role of $n-3$ fatty acids was studied very carefully. IMMIDIET study population was used for this analysis.

Italians showed lower plasma and RBC n-3 levels as compared to Belgians and English. Reduced Rank Regression analysis indicated two one factor-dietary patterns explaining the total variation of the sum of plasma and $\mathrm{RBC} n-3$, respectively. In a holistic dietary analysis, neither fish nor mollusks intake seemed to contribute to $n-3$ variation as compared to vegetable oils and polyphenols-rich food.

The present conclusions stress the importance of adherence to a dietary pattern including vegetable oils and polyphenols-rich food in establishing better $n-3$ fatty acids profile. This is another example indicating the importance of studying also in holistic way through dietary patterns the association of human diet with CVD related biomarkers.

- Are all different approaches to dietary analyses useful in the study of human diet and CVD risk factors and other metabolic parameters? 
Via this Thesis 5 different approaches to dietary analyses were studied using 3 sample sets (Moli-sani and IMMIDIET study and a clinical trial). All of them provided valuable conclusions in the study of human diet in association to CVD risk factor or other metabolic parameters; however, the kind of messages derived from each of them was different.

The development of FAC-score gave the opportunity for overall assessment of antioxidant food intake, a critical aspect of human diet, in association with inflammation process, hypertension and HRQL. By this methodology the co-linearity problem of dietary data was overcome. In addition, public health managers could be informed about the antioxidant food intake status of the reference population and establish its impact on CVD related health outcomes.

Further study focused on weekly mushroom and selenium intake, an antioxidant food and nutrient, correspondingly. Their consumptions were independently and positively associated with glucose levels. By this methodology scientists could make public health statements on the importance of specific antioxidant food or nutrient intake to the reference population.

The adherence to dietary patterns including high consumption of folate food sources or vegetable oils and polyphenols-rich food was associated with the presence of better blood folate or n-3 fatty acids profile, correspondingly. The holistic "a posteriori" dietary analyses provided messages supporting the importance of food consumption in the context of dietary pattern adherence to establish better CVD related biomarkers profile.

The latter was also concluded from a clinical trial, which demonstrated that supplementation with natural folate-rich foods, folic acid and 5-MTHF, reached a similar reduction in homocysteine concentrations. This illustrates again the importance of natural food sources consumption in establishing adequate levels of CVD related biomarkers.

All these inferences stress the usefulness of all methodological approaches to dietary analyses and point out that their application should be done according to the aim of the study. Also the use of multiple methodologies in dietary analysis may help to establish more integrated conclusions about the association of human diet and CVD risk factors or other metabolic parameters. 


\section{Chapter 9. Conclusions}

The main messages for the healthy adult population derived through this Thesis could be summarized as follow:

- In men the choice of foods high rather than low in antioxidant content may reduce blood pressure and inflammation.

- FAC-score as a dietary index could describe the antioxidant food intake of the general population. It may be considered as a useful tool in the study of the association of antioxidant vitamins and phytochemicals intake and CVD related health outcomes.

- The total antioxidant content of diet evaluated through FAC-score could independently explain the association of HRQL with Mediterranean pattern adherence.

- FAC-score could also be associated with other CVD disease risk factors, not necessarily clinically evaluable.

- Both mushroom intake and dietary selenium were independently and positively associated with blood glucose. The study of the association of human diet with CVD risk factors should also focused on food or nutrient intake.

- Dietary patterns adherence including the consumption of natural food sources of folate could assure adequate serum levels of folate. The dietary patterns extraction as a holistic approach in dietary analysis could derive valuable conclusions.

- Consumption of food naturally rich in folate is a valuable approach to decrease homocysteine levels as compared to supplemental folate intake.

- The study of single supplemental nutrient intake through clinical trials may not be enough in establishing associations between diet and CVD related biomarkers.

- Adherence to a dietary pattern including vegetable oils and polyphenols-rich food could establish better $n-3$ blood fatty acids profile. Another example of holistic dietary analysis in association with CVD related biomarkers.

- All methodological approaches to dietary analyses provided valuable conclusions in the study of human diet in association to CVD risk factor or other metabolic parameters. Their application should be done according to the aim of the study and the use of multiple methodologies may help to establish more integrated conclusions. 
- Despite the importance of the Thesis limitations still exist. The cross-sectional designs of Moli-sani and IMMIDIET studies do not enable determination of causality. These studies were not aimed at investigating biological mechanisms and further studies are necessary to investigate the possible, underlined physiological mechanisms. In both studies FFQs were used which do not allow the precise evaluation of each specific nutrient investigated. Possible errors by misreporting in the evaluation of the dietary habits of each individual should also be acknowledged. The application of different methodological approaches in dietary analysis was limited with major concern to antioxidant vitamins and phytochemicals, selenium, folate and $n-3$ and $n-6$ fatty acids intakes. The generalization of the present conclusions to other nutrients or food intake is difficult. All present associations have not been well evaluated in other study samples. This is limiting the generalization of our conclusions and makes the confirmation of present results by other studies important.

- Further study of the association of dietary habits with cardiovascular risk factors using different methodological approaches in dietary analysis is still needed. The use of different study designs and statistical methods and may extent the present conclusions. The confirmation of these results applying dietary analysis with main interest other than antioxidant vitamins and phytochemicals and folate intake is also essential. 
Dietary habits have been strongly associated with primary and secondary prevention of CVD. The evaluation of dietary habits can be performed at many levels leading to different types of public health conclusions. Dietary indexes, food and nutrient intake assessment, dietary pattern extraction and clinical trial are a summary of the different methodological procedures. Intergraded nutritional analysis is of crucial importance especially when the association of dietary habits with chronic diseases such as CVD is studied.

This thesis aimed at examining the complex impact of dietary habits on major cardiovascular risk factors and other metabolic parameters of free-living populations, using different methodological approaches to dietary analysis, in order to get more integrated conclusions.

Considering the use of dietary indexes, FAC-score was developed with main aim the evaluation of antioxidant food intake in healthy population. It was revealed that in men the choice of foods high rather than low in antioxidant content evaluated through FAC-score might reduce blood pressure and inflammation. In addition, FAC-score could independently explain the association of HRQL with Mediterranean pattern adherence. This dietary index may be considered as a useful tool in the study of the association of antioxidant vitamins and phytochemicals intake and CVD related health outcomes.

Except the holistic methodological approaches in the dietary analysis food and nutrient intake assessment could derive valuable conclusions. In particular, both mushroom intake and dietary selenium were independently and positively associated with blood glucose in a healthy free-living population.

Moreover, dietary patterns extraction indicated patterns including the consumption of natural food sources of folate that was associated with adequate serum levels of folate. The importance of consumption of natural food sources of folate was confirmed by a clinical trial. It concluded that consuming food naturally rich in folate is a valuable approach to decrease homocysteine levels.

The study of single supplemental nutrient intake through clinical trials may not be enough in establishing associations between diet and CVD related biomarkers. In contrary, dietary extraction could result valuable conclusions. In the present Thesis dietary pattern analysis indicated that the higher adherence to patterns including vegetable oils and polyphenolsrich food could establish better $n-3$ blood fatty acids profile analysis.

This Thesis could point out that all methodological approaches to dietary analyses are useful in the study of human diet in association with CVD risk factors and other metabolic parameters. Their application should be done according to the aim of the study while the use of multiple methodologies may help to establish more integrated conclusions. 


\section{Chapter 11. Curriculum Vitae}

\section{Georgios Pounis, MSc \\ Dietician - Nutritionist, Biostatistician}

\section{Personal details}

- Date of Birth: October 6th, 1985

- Marital Status: Single

- Address: 6 Krinis St, Kypseli, 11362, Athens, Greece

- Telephone: +30-210 9821084 (home) +30-694 4572000 (mobile)

- Email: gdpounis@moli-sani.org

\section{Education}

- 1997-2003: 15th High School of Athens

- 2004-2008: BSc in Dietetics and Nutrition, Department of Science of Dietetics - Nutrition, Harokopio University, Athens, Greece

- 2008-2010 MSc in Biostatistics, School of Medicine and Dept of Mathematics, National and Kapodostirian University of Athens, Athens, Greece

- January 2011 until January 2014 (scheduled time of thesis presentation): PhD thesis to be presented to CARIM School for Cardiovascular Diseases, Maastricht University, The Netherlands.

Title: The impact of dietary habits on cardiovascular disease risk factors and other bioclinical characteristics of free living population: practice of different methodological approaches on dietary analysis.

\section{Professional experience}

- 2012-2013: Research associate, Hellenic Health Foundation, Athens, Greece

- 2011-2013: Visiting Scientist, Laboratori di Ricerca, Fondazione di Ricerca e Cura "Giovanni Paolo II", Campobasso, Italy.

- 2013 (June)-present: Visiting Scientist at the Department of Epidemiology and Prevention, IRCCS Istituto Neurologico Mediterraneo NEUROMED, 86077 Pozzilli (Isernia), Italy

- 2010: Research associate, Department of Science of Dietetics - Nutrition, Harokopio University, Athens, Greece

- 2008-2010: Research associate, Nutrition Unit, Department of Food Science, Agricultural University of Athens, Athens, Greece.

- 2008: Instructor in training programs with applied issues to Nutrition and Dietetics.

- 2008-2013: Director of dietetic counseling office in Athens.

\section{Publications}

20 publications in peer reviewed international journals, $\mathrm{h}$-index (calculated via Scopus) $=6$

\section{Publications in congresses}

- 2006 until now: 11 oral presentations in Greek and International Congresses.

- 2006 until now: 25 poster presentations in Greek and International Congresses.

\section{Honors - Awards}

- 2006: 3rd Research Award, 10th Congress of Hellenic Atherosclerosis and Lipidology Society, Athens, for his work on "Relationship of dietary patterns with hypertension, hypercholesterolemia and obesity in remote Greek rural populations: The "ARGO" study".

- 2007: 3rd Research Award in 9th Conference on Nutrition - Dietetics, Hellenic Dietetic Association, Athens, for his work on "Long-term consumption of fish and omega-3 fatty acids is associated with reduced depressive behavior in patients with acute coronary syndrome". 


\section{Chapter 12. Publications}

1. Pounis G, Costanzo S, Persichillo M, de Curtis A, Sieri S, Vinceti M, Zito F, Di Castelnuovo $\mathrm{AF}$, Donati MB, de Gaetano G, lacoviello L, on behalf of the Moli-sani Project Investigators. Mushroom consumption and dietary selenium intake in association with fasting glucose levels in a free-living Italian adult population: the Moli-sani study. Diabetes \& Metabolism 2013. pii: S1262-3636(13)00180-8. doi: 10.1016/j.diabet.2013.09.007

2. Pounis G, Di Castelnuovo AF, de Lorgeril M, Krogh V, Siani A, Arnout J, Cappuccio FP, van Dongen M, Zappacosta B, Donati MB, de Gaetano G, lacoviello L, on behalf of the European Collaborative Group of the IMMIDIET Project. Folate intake and folate serum levels in men and women from two European populations: The IMMIDIET project. Nutrition, In Press.

3. Pounis G, de Lorgeril M, Salen P, Laporte F, Krogh V, Siani A, Arnout J, Cappuccio FP, van Dongen M, Donati MB, de Gaetano G, lacoviello L, on behalf of the European Collaborative Group of the IMMIDIET Project. Dietary patterns and blood and tissue fatty acids levels of 3 European populations. Nutr Met Card Dis, In Press.

4. Bonaccio M, Di Castelnuovo A, Bonanni A, Costanzo S, De Lucia F, Pounis G, Zito F, Donati MB, de Gaetano G, lacoviello L; Moli-sani project Investigators. Adherence to a Mediterranean diet is associated with a better health-related quality of life: a possible role of high dietary antioxidant content. BMJ Open. 2013 Aug 13;3(8). pii: e003003. doi: 10.1136/bmjopen-2013-003003.

5. Zappacosta B, Mastroiacovo P, Persichilli S, Pounis G, Ruggeri S, Minucci A, Carnovale E, Andria G, Ricci R, Scala I, Genovese O, Turrini A, Mistura L, Giardina B, lacoviello L. Homocysteine lowering by folate-rich diet or pharmacological supplementations in subjects with moderate hyperhomocysteinemia. Nutrients. 2013 May 8;5(5):1531-43. doi: 10.3390/nu5051531.

6. Pounis G, Costanzo S, di Giuseppe R, de Lucia F, Santimone I, Sciarretta A, Barisciano P, Persichillo M, de Curtis A, Zito F, Di Castelnuovo AF, Sieri S, Benedetta Donati M, de Gaetano G, lacoviello L. Consumption of healthy foods at different content of antioxidant vitamins and phytochemicals and metabolic risk factors for cardiovascular disease in men and women of the Moli-sani study. Eur J Clin Nutr. 2013 Feb;67(2):20713. doi: 10.1038/ejen.2012.201.

7. Argyri K, Theophanidi E, Kapna A, Staikidou C, Pounis G, Komaitis M, Georgiou C, Kapsokefalou M. Iron or zinc dialyzability obtained from a modified in vitro digestion 
procedure compare well with iron or zinc absorption from meals. Food Chem. $2011 \mathrm{Jul}$ 15;127(2):716-21. doi: 10.1016/j.foodchem.2011.01.005.

8. Farajian P, Risvas G, Karasouli K, Pounis GD, Kastorini CM, Panagiotakos DB, Zampelas A. Very high childhood obesity prevalence and low adherence rates to the Mediterranean diet in Greek children: the GRECO study. Atherosclerosis. 2011 Aug;217(2):525-30. doi: 10.1016/j.atherosclerosis.2011.04.003.

9. Magriplis E, Farajian P, Pounis GD, Risvas G, Panagiotakos DB, Zampelas A. High sodium intake of children through 'hidden' food sources and its association with the Mediterranean diet: the GRECO study. J Hypertens. 2011 Jun;29(6):1069-76. doi: 10.1097/HJH.0b013e328345ef35.

10. Yannakoulia M, Tyrovolas S, Pounis G, Zeimbekis A, Anastasiou F, Bountziouka V, Voutsa K, Gotsis E, Metallinos G, Lionis C, Polychronopoulos E, Panagiotakos D. Correlates of low dietary energy reporting in free-living elderly: the MEDIS study. Maturitas. 2011 May;69(1):63-8. doi: 10.1016/j.maturitas.2011.01.016.

11. Sialvera TE, Pounis GD, Koutelidakis AE, Richter DJ, Yfanti G, Kapsokefalou M, Goumas G, Chiotinis N, Diamantopoulos E, Zampelas A. Phytosterols supplementation decreases plasma small and dense LDL levels in metabolic syndrome patients on a westernized type diet. Nutr Metab Cardiovasc Dis. 2012 Oct;22(10):843-8. doi: 10.1016/j.numecd.2010.12.004.

12. Polychronopoulos E, Bountziouka V, Zeimbekis A, Tsiligiani I, Pounis G, Gotsis E, Metallinos G, Lionis C, Panagiotakos DB. Dietary fats in relation to depressive symptoms by cardiovascular disease risk factors status of elderly people living in Mediterranean islands. Int J Food Sci Nutr. 2010 Nov 2. [Epub ahead of print]

13. Pounis GD, Tyrovolas S, Antonopoulou M, Zeimbekis A, Anastasiou F, Bountztiouka V, Metallinos G, Gotsis E, Lioliou E, Polychronopoulos E, Lionis C, Panagiotakos DB. Longterm animal-protein consumption is associated with an increased prevalence of diabetes among the elderly: the Mediterranean Islands (MEDIS) study. Diabetes Metab. 2010 Dec;36(6 Pt 1):484-90. doi: 10.1016/j.diabet.2010.06.007.

14. Tyrovolas S, Pounis G, Bountziouka V, Polychronopoulos E, Panagiotakos DB. Repeatability and validation of a short, semi-quantitative food frequency questionnaire designed for older adults living in Mediterranean areas: the MEDIS-FFQ. J Nutr Elder. 2010 Jul;29(3):311-24. doi: 10.1080/01639366.2010.499096.

15. Mamplekou E, Bountziouka V, Psaltopoulou T, Zeimbekis A, Tsakoundakis N, Papaerakleous N, Gotsis E, Metallinos G, Pounis G, Polychronopoulos E, Lionis C, 
Panagiotakos D. Urban environment, physical inactivity and unhealthy dietary habits correlate to depression among elderly living in eastern Mediterranean islands: the MEDIS (MEDiterranean ISlands Elderly) study. J Nutr Health Aging. 2010 Jun;14(6):44955.

16. Tyrovolas S, Pounis G, Zeimbekis A, Antonopoulou M, Bountziouka V, Gotsis E, Metallinos G, Polystipioti A, Polychronopoulos E, Lionis C, Panagiotakos DB. Associations of energy intake and type 2 diabetes with hypertryglyceridemia in older adults living in the Mediterranean islands: the MEDIS study. J Nutr Elder. 2010 Jan;29(1):72-86. doi: 10.1080/01639360903574668.

17. Polychronopoulos E, Pounis G, Bountziouka V, Zeimbekis A, Tsiligianni I, Qira BE, Gotsis E, Metallinos G, Lionis C, Panagiotakos D. Dietary meat fats and burden of cardiovascular disease risk factors, in the elderly: a report from the MEDIS study. Lipids Health Dis. 2010 Mar 18;9:30. doi: 10.1186/1476-511X-9-30.

18. Tyrovolas S, Psaltopoulou T, Pounis G, Papairakleous N, Bountziouka V, Zeimbekis A, Gotsis E, Antonopoulou M, Metallinos G, Polychronopoulos E, Lionis C, Panagiotakos DB. Nutrient intake in relation to central and overall obesity status among elderly people living in the Mediterranean islands: the MEDIS study. Nutr Metab Cardiovasc Dis. 2011 Jun;21(6):438-45. doi: 10.1016/j.numecd.2009.10.012.

19. Tyrovolas S, Zeimbekis A, Bountziouka V, Voutsa K, Pounis G, Papoutsou S, Metallinos G, Ladoukaki E, Polychronopoulos E, Lionis C, Panagiotakos DB. Factors Associated with the Prevalence of Diabetes Mellitus Among Elderly Men and Women Living in Mediterranean Islands: The MEDIS Study. Rev Diabet Stud. 2009 Spring;6(1):54-63. doi: 10.1900/RDS.2009.6.54.

20. Pounis GD, Panagiotakos DB, Chrysohoou C, Aggelopoulos P, Tsiamis E, Pitsavos C, Stefanadis C. Long-term fish consumption is associated with lower risk of 30-day cardiovascular disease events in survivors from an acute coronary syndrome. Int J Cardiol. 2009 Aug 21;136(3):344-6. doi: 10.1016/j.ijcard.2008.04.063.

\section{H-index (calculated via Scopus) $=6$}




\section{Chapter 13. Acknowledgments}

It is a pleasure to thank all the people who made this thesis possible.

I am grateful to Prof. Coen Hemker and Prof. Hugo ten Cate who accepted me as a PhD student and made possible a truly European experience to a Greek student preparing his thesis in Italy in collaboration with a French investigator to be presented and discussed in The Netherlands.

I would not have achieved this ambitious goal without the guidance and mentoring of Prof. Giovanni de Gaetano, Prof. Maria Benedetta Donati and Prof. Licia lacoviello whose expertise, understanding, and patience added considerably to my research career and PhD experience. Their exceptional hospitality made my stay in Italy more than comfortable and productive as well.

I wish to thank also Prof. Michel de Lorgeril for his advice and support.

I want to extend my sincere gratitude to my colleagues and friends of the Research Laboratories in Campobasso who supported me from my first visit to Italy.

My final thoughts go to my family and Eleftheria, I would not have gone so far without their love and encouragement.

Georgíos 